\title{
COMPUTATIONAL STUDY OF THE EFFECTS OF \\ PROTRUDING STUDS CASING TREATMENT ON \\ THE PERFORMANCE OF AN AXIAL
}

TRANSONIC TURBOFAN

By

MAX DAVID COLLAO

Robert S. Webster

Research Associate Professor of

Computational Engineering

(Chair)

James C. Newman III

Professor of Computational Engineering (Committee Member)
Kidambi Sreenivas

Research Professor of Computational

Engineering

(Committee Member)
Matt Matthews

Associate Dean of the College of Arts and Sciences

(Committee Member) 


\section{COMPUTATIONAL STUDY OF THE EFFECTS OF \\ PROTRUDING STUDS CASING TREATMENT ON \\ THE PERFORMANCE OF AN AXIAL \\ TRANSONIC TURBOFAN}

By

MAX DAVID COLLAO
A Dissertation Submitted to the Faculty of the University of Tennessee at Chattanooga in Partial Fulfillment of the Requirements of the Degree of Doctor of Philosophy in Computational Engineering

The University of Tennessee at Chattanooga Chattanooga, Tennessee

May 2017 
Copyright (C) 2017

\section{By MAX DAVID COLLAO}

All Rights Reserved 


\begin{abstract}
Turbo-compressors play a crucial role in the operation of air-breathing engines and special attention is given to the compressor's ability to withstand adverse operating conditions. Various methods have been devised in order to enhance compressor stability and increase compressor stall margin, usually with some sacrifice of performance and efficiency. Active methods make use of external devices injecting and/or bleeding air from the compressor duct. Passive methods are primarily based on casing treatments, often involving flow paths into the rotor casing, to affect the behavior of the flow in favorable ways. In either case, it is desirable that the stability enhancing method be as simple as possible and that it keep performance and/or efficiency degradation to a minimum.
\end{abstract}

The present work consists of a CFD analysis performed to evaluate the impact of a novel form of passive stability control on the operation of an axial flow fan stage. The casing treatment consists of "studs," which are solid structures protruding from the casing into the duct. These structures are located slightly downstream of the fan rotor trailing edge. The stage that is studied in this investigation is the NASA SDT2-R4 transonic turbofan. The simulation software used is “Tenasi," an in-house developed flow solver.

Various simulations were conducted of the turbofan operation with and without casing treatment. Measures of performance and efficiency of the turbofan stage with no casing treatment were computed, and the results showed good agreement with available experimental data. The 
computed results from simulation with casing treatment suggest that protruding studs have the potential to improve the stability of the turbofan. The computed stall margin increase using an initial configuration of protruding studs is about $5.4 \%$ in terms of mass flow rate, with a decrease in efficiency of about $1.6 \%$. The flow-field was investigated through visualizations of flow features in order to understand the mechanisms of flow instabilities and stability enhancement. Other simulations were also carried out using modifications of the original studs to assess further changes in performance. 


\section{DEDICATION}

I dedicate this work to David, my natural inner child. 


\section{ACKNOWLEDGEMENTS}

First and foremost, I would like to thank God. Because He loved me first before I loved Him.

I am very grateful to Dr. Robert S. Webster for allowing me to work with him, for sharing his expertise in the field of turbomachinery and for serving as my adviser throughout my time doing research. I would like to thank Dr. Kidambi Sreenivas for his support and his ideas in carrying forward simulations and CFD analysis. I would like to thank Dr. James C. Newman III for serving in my committee and for serving as a leader at the SimCenter. I would like to say thanks to Dr. Matt Matthews for serving in my committee and for his great dedication as a math professor. I would also like to thank Dr. Timothy W. Swafford, Dr. Reinhold C. Mann, and Dr. Steven L. Karman Jr for their service as leaders and mentors.

Finally, I will always be thankful to my friends and family, both in the United States and in Peru. Their cheer and support was critical during my most difficult times. You all are a blessing! Thank you guys! 


\section{TABLE OF CONTENTS}

ABSTRACT

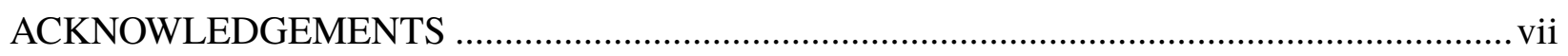

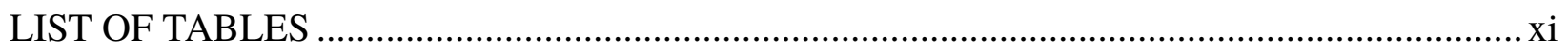

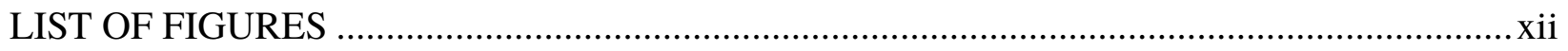

CHAPTER

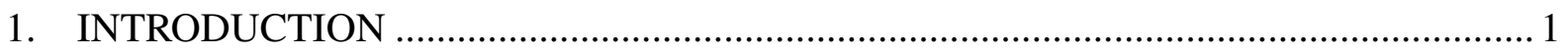

$1.1 \quad$ What about compression systems? ………………............................................ 1

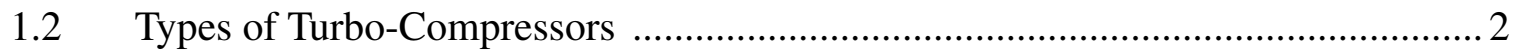

$1.3 \quad$ Instabilities in Axial Compressors ……………................................................ 5

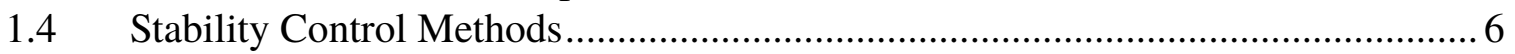

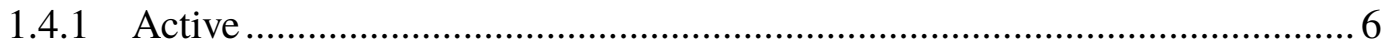

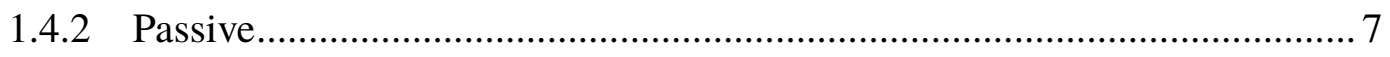

1.5 CFD Application to Turbo-Machinery ............................................................. 8

2. MATHEMATICAL DESCRIPTION OF THE FLOW ................................................. 9

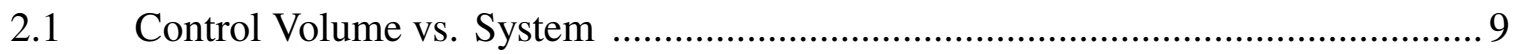

2.2 Equations Describing the Fluid Flow …………........................................... 12

2.2.1 Conservation of Mass ………………................................................ 12

2.2.2 Conservation of Momentum …………………................................ 13

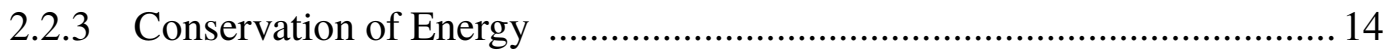

2.2.4 Thermodynamic Relations for Perfect Gas ............................................. 15

2.2.5 Complete System in Vector Form ………............................................ 16 
3. COMPRESSION SYSTEM AND CASING TREATMENT

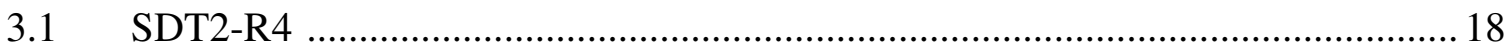

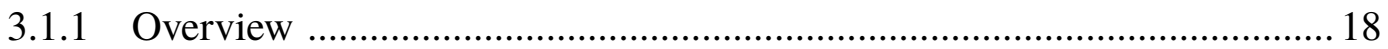

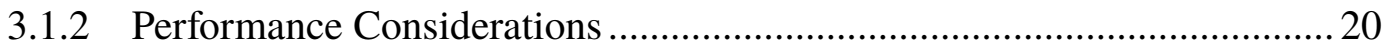

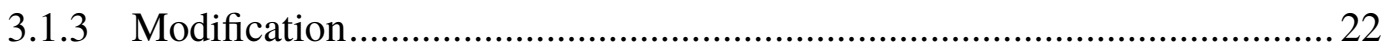

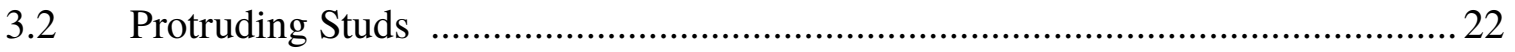

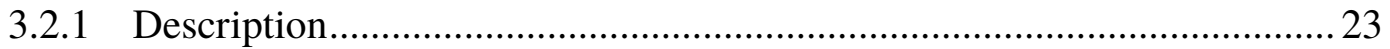

3.2.1.1 Elongated "U" Shape ……………………................................. 23

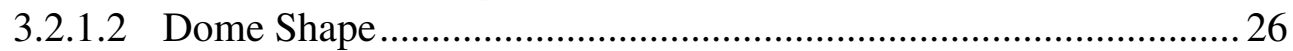

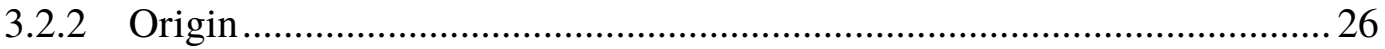

3.2.3 Search for Similar Form of Casing Treatment .......................................... 29

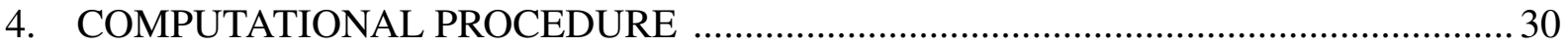

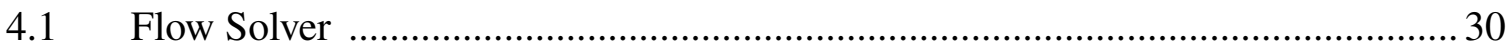

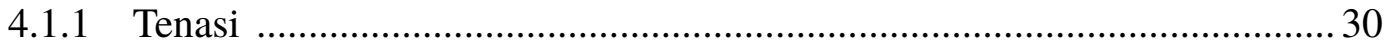

4.1.2 Aspects of Numerical Solution …………………................................ 31

4.1.2.1 Spatial Discretization ................................................................. 31

4.1.2.2 Temporal Discretization ............................................................. 32

4.1.2.3 Turbulence Modelling ………………………................................ 33

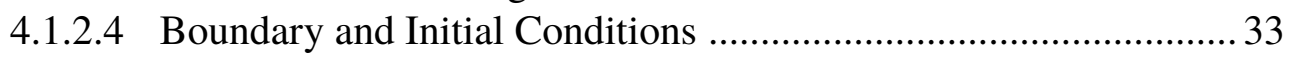

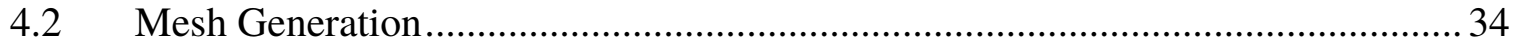

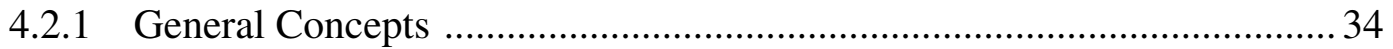

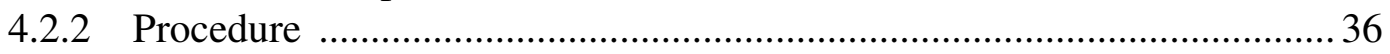

4.2.3 Detail of Interpolative Interface and Studs Volume Mesh......................... 40

4.2.4 Finalizing the Mesh ............................................................................... 43

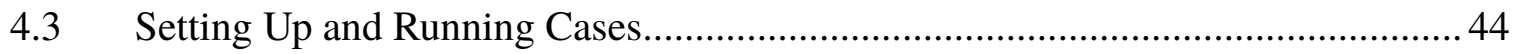

$4.4 \quad$ Post-Processing and Visualization ....................................................................... 46

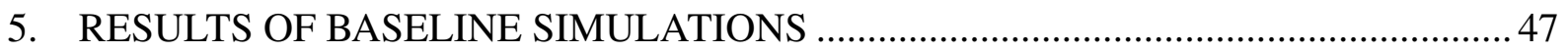

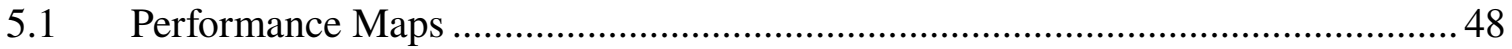

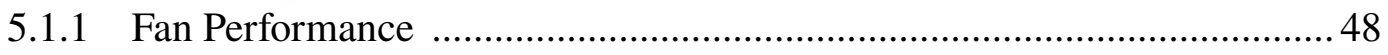

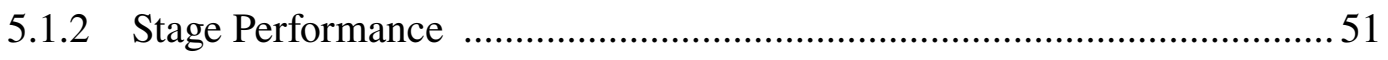

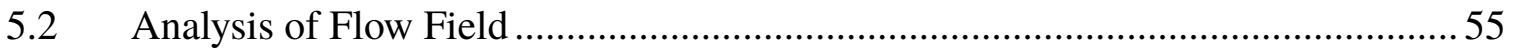

5.2.1 Location of Shocks by Operating Condition ..........................................5 55

5.2.2 Low Momentum Regions by Operating Condition ...................................... 62

5.2.3 Instability Region in Near-Stall Operating Condition.................................65

5.3 Influence of the Mesh on the Solutions ............................................................... 73 
6. CASING TREATMENT SIMULATION

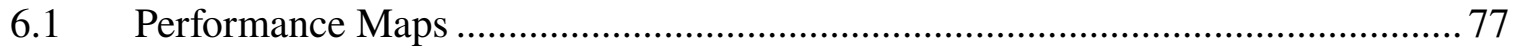

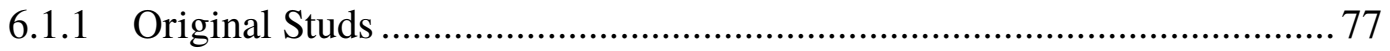

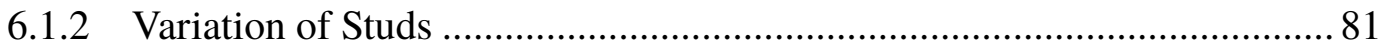

6.1.2.1 Performance by Levels of Protrusion ............................................. 82

6.1.2.2 Half Extension and Dome Shaped Studs ...................................... 85

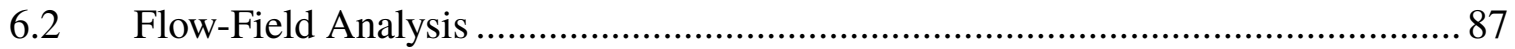

6.2.1 Radial Distribution of Axial Flow Velocity ................................................ 87

6.2.2 Radial Distribution of Relative Flow Angles........................................... 97

6.2.3 Flow-Field Features at $131.7 \mathrm{kPa}$ Back Pressure ..................................... 99

6.2.4 Flow-Field Features at New Near-Stall Conditions With Casing

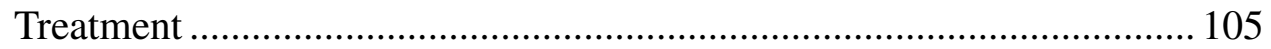

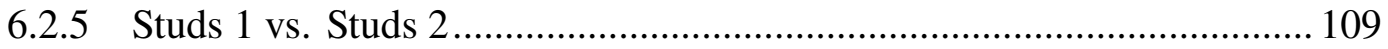

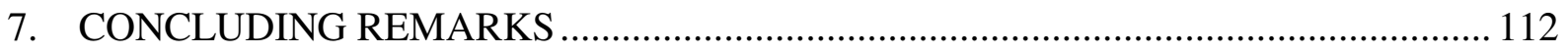

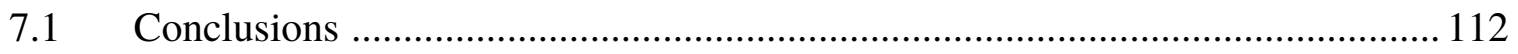

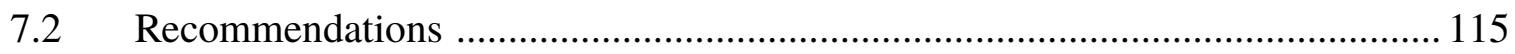

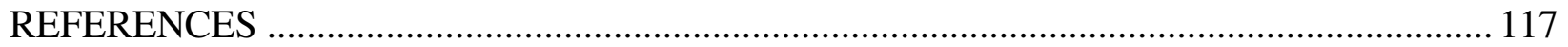

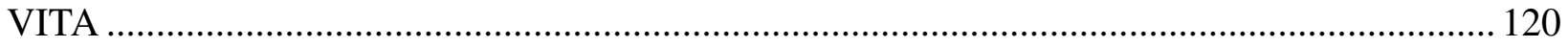




\section{LIST OF TABLES}

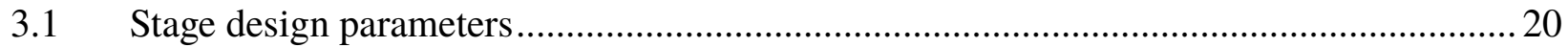

6.1 Change in performance across results for eleven tested back pressures. ....................... 80 


\section{LIST OF FIGURES}

1.1 Various types of compressors [4] in (a) a TF30-P-6 Pratt \& Whitney engine, (b) a Lycoming T53 turboshaft engine, and (c) a Saturn MD-120 engine...

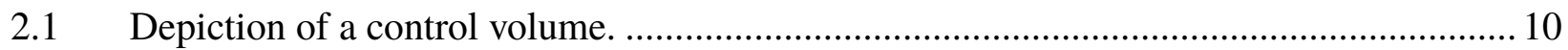

2.2 Relation between systems and control volumes [1] ...................................................... 12

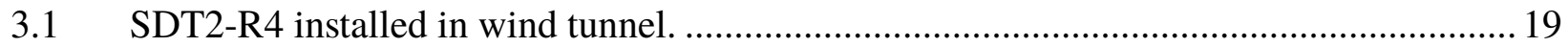

3.2 Experimentally measured performance curves for the SDT2-R4 stage [2] .....................2 21

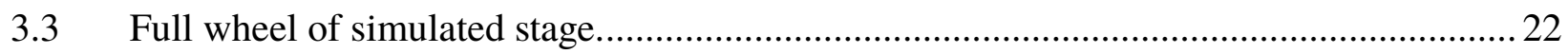

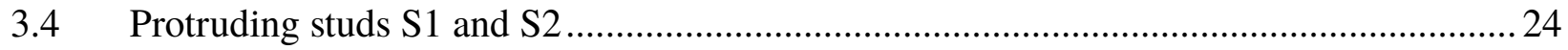

3.5 Dimensions as percentage of fan chord length at tip, alignment, and location of studs....25

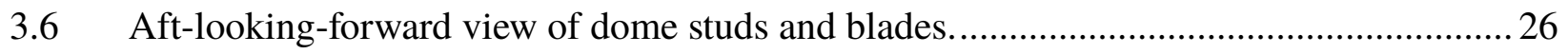

3.7 Protruding rings from Lin's work [3] (including caption from original text)...................27

3.8 Performance curve using trailing edge ring [3] ............................................................ 28

4.1 Minimum and maximum point spacing used in different regions of the turbofan stage.... 36

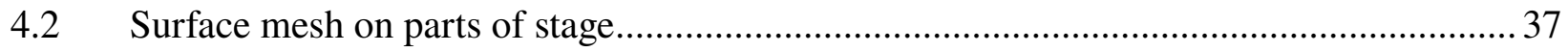

4.3 Plane cut of volume mesh near a corner. .................................................................... 38

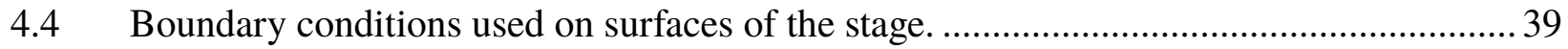

4.5 The two sliding interfaces used in earlier simulations are shown in pink, rotor blades and stator vanes are shown in light blue, and casing is shown in grey. 
4.6 Surfaces separating the Rotor, Stator, and Studs volumes.

4.7 Aft-looking-forward view of volume mesh after generation and merging where the casing surface is shown in green, rotor blades and studs are depicted in pink, and a plane cut of the volume mesh is depicted in grey.

4.8 Sample of mass flow computed across four boundaries in three consecutive simulations.

5.1 Planes of integration of mass flow, total pressure and total temperature ....................... 48

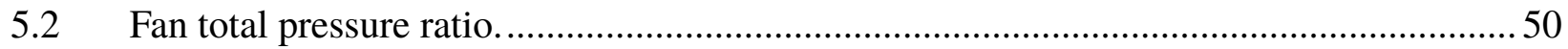

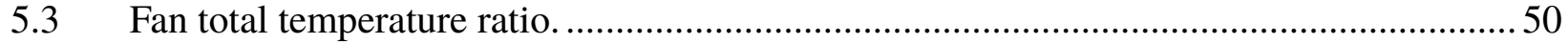

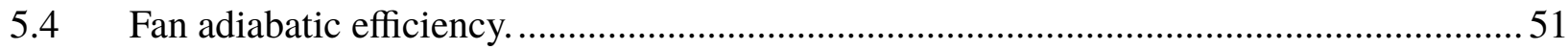

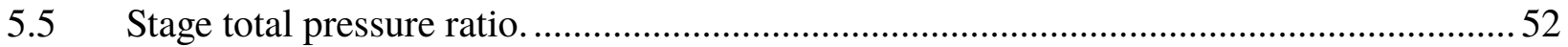

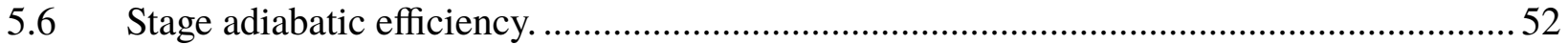

5.7 Temperature ratio across stage for choked flow conditions, peak efficiency, and near stall at $100 \%$ speed.

5.8 Mass imbalances at solution termination where the values are presented as a percentage of mass flow through passage.

5.9 Stage surfaces colored by static pressure at different flow conditions...........................56

5.10 Radial cut at $60 \%$ span colored by density (in $\mathrm{kg} / \mathrm{m}^{3}$ ) for three back pressure conditions.

5.11 Radial cut at $95 \%$ span colored by density (in $\mathrm{kg} / \mathrm{m}^{3}$ ) for three back pressure conditions

5.12 Radial cut at $98 \%$ span colored by density (in $\mathrm{kg} / \mathrm{m}^{3}$ ) for three back pressure conditions.

5.13 Stream lines and radial cuts of solution field colored by relative Mach number.

5.14 Relative velocity vectors. 
5.15 Forward-looking-aft view of constant x planes near low Mach number region.

5.16 Low momentum region near casing for baseline case near stall................................66

5.17 Forward-looking-aft view of parallel-to-shock plane cuts of low momentum region for baseline case near stall.

5.18 Low momentum region as exposed by $v$ isosurface.

5.19 Tip gap flow patterns for baseline case near stall................................................. 71

5.20 Flow pattern around stator vanes for baseline case near stall. ................................... 72

5.21 Comparing performance maps obtained when using a maximum of 29 viscous layers in the computational mesh.

5.22 Performance curves obtained in earlier stages of this work where a maximum of 29 viscous layers and a different surface mesh were used.

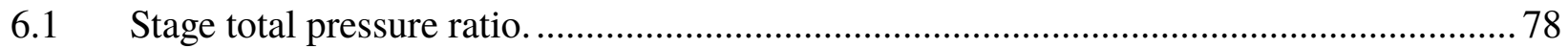

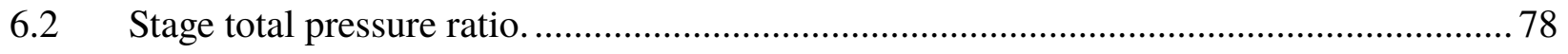

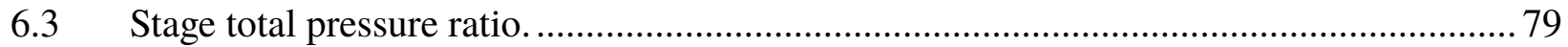

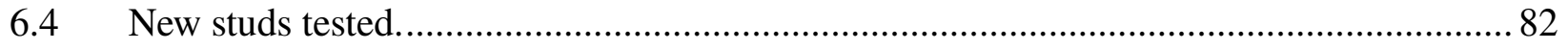

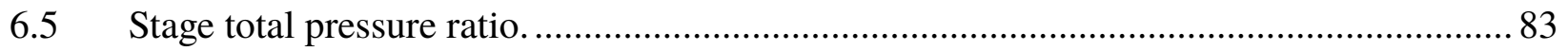

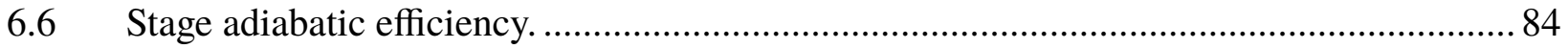

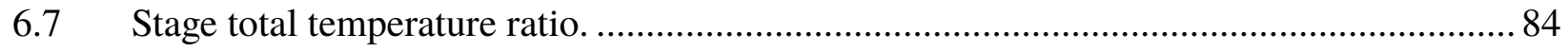

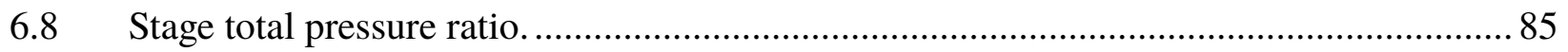

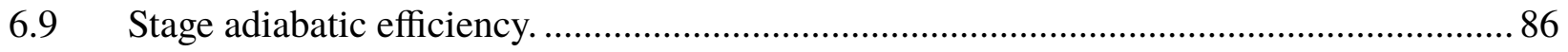

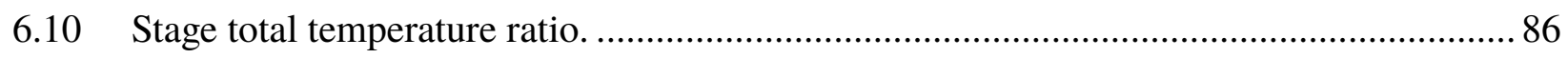

6.11 Axial location of planes, where radial distribution samples were taken, displaying studs near the trailing edge of the rotor blades. ............................................... 88

6.12 Radial distribution of axial velocities at computed peak efficiency.............................90 
6.13 Radial distribution of axial velocities at $131 \mathrm{kPa}$ back pressure.

6.14 Comparison of axial momentum at a $75 \%$ fan blade span (78\% outlet plane span) radial cut at $131 \mathrm{kPa}$ back pressure.

6.15 Radial distribution of axial velocities at baseline computed near stall conditions.

6.16 Comparison of axial momentum at a $75 \%$ fan blade span (78\% outlet plane span) radial cut at $131.7 \mathrm{kPa}$ back pressure........................................................... 96

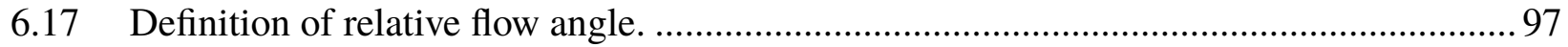

6.18 Comparison of relative flow angles at outlet plane.............................................. 98

6.19 Comparison of relative Mach number in passages between baseline and casing treatment simulations, where the cuts are located at mid height of the studs (97.8\% span)

6.20 Isosurface of $v$ side view comparison....... 102

6.21 Patterns of tip gap flow using casing treatment at $131.7 \mathrm{kPa}$ back pressure 104

6.22 Recirculation on suction side of stator blades near casing for $131.7 \mathrm{kPa}$ back pressure. . 105

6.23 Forward-looking-aft view of isosurfaces of $v$ at near stall conditions using Studs 1 and Studs 2 .

6.24 Patterns of tip gap flow using treatment at near stall conditions 108

6.25 Recirculation on suction side of stator blades near casing for near stall conditions

6.26 Comparison of total temperature coloration across Studs 1 and Studs 2, where the radial cuts are located at mid height of the studs (97.8\% span).

6.27 Comparison of velocity vectors around Studs 1 and Studs 2, where the radial cuts at mid height of the studs ( $97.8 \%$ span).

6.28 Flow patterns around Studs 


\section{CHAPTER 1}

\section{INTRODUCTION}

The work presented in this dissertation consists of a study of the performance effects caused by using studs protruding off of the casing in a turbofan stage. The study was performed using computational tools, and its main goal was that of exploring relatively simple methods for enhancing the flow stability in compression systems. The layout of the manuscript is as follows: Chapter I is an introductory overview of compression systems and the instabilities related to them. Chapter II provides a mathematical description of the fluid flow. Chapter III provides a description of the turbo-machine and the type of casing treatment under study. Chapter IV introduces the flow solver and describes the computational procedure that made up a great part of this work. Chapter $\mathrm{V}$ presents the validation of the CFD tools by comparing baseline simulation results with available experimental data. Chapter VI presents the results of simulating the operation of the turbo-machine at hand with the casing treatment, along with an analysis of the changes. Chapter VII gives concluding remarks and recommendations.

\subsection{What about compression systems?}

Gas compressors are present in diverse engineering applications that simplify and enhance the lives of people. From the most ubiquitous applications, such as refrigerators, to the most specialized ones, like power generation, there is frequently a need for pressurizing a gas in order to 
later take advantage of the energy it stores or to feed combustion processes. Hence the importance of compression systems.

One application where compression systems are important is air breathing engines for aircraft propulsion. Actually, the role of a compression system in aircraft propulsion is beyond necessary but also critical. This is because in addition to playing a crucial role in thrust generation, the integrity of the entire propulsion system weighs heavily on the compressor's ability to withstand adverse flight conditions that affect air intake negatively.

Compression systems in air breathing engines comprise a certain kind of turbo-machinery called turbo-compressors. In general, turbo-machines are rotating devices that interact with a fluid through blades attached to a shaft. More specifically, turbo-compressors absorb shaft work and transfer mechanical energy to continuously flowing air in order to raise its total enthalpy. In doing so, the air is moved through the duct of the compressor at various speeds (depending on its location and the components with which it interacts), and it behaves (its properties will change) in different ways.

Turbo-compressors possess features that make them suitable for engines if high power-toweight ratios are required. Compared to other forms of compressors, they are able to displace large amounts of air in continuous flow. Furthermore, since they possess no reciprocating components, the problem of shaft balancing is less likely to occur, which makes turbo-compressors more reliable.

\subsection{Types of Turbo-Compressors}

Using a cylindrical frame of reference, a classification can be based on the direction of the main flow as projected on the meridional plane, $z-r$, of the turbo-machine. If the direction of 
the flow remains mainly parallel to the axis of rotation, the compression system is called "axial" (Figure 1.1a). If the flow changes direction through the rotor's flow path from the axial direction to the radial direction, the compressor is called "centrifugal" (Figure 1.1b). If the flow path of the rotor is more or less diagonal, then it is a "mixed-flow" compressor (Figure 1.1c). The type of compression system used in this work is of axial flow. Specifically, the turbo-machine used here is the transonic turbofan SDT2-R4, which will be further described in Chapter 4. Through the rest of this chapter, the focus will be placed on flow features and stability of axial compressors. 


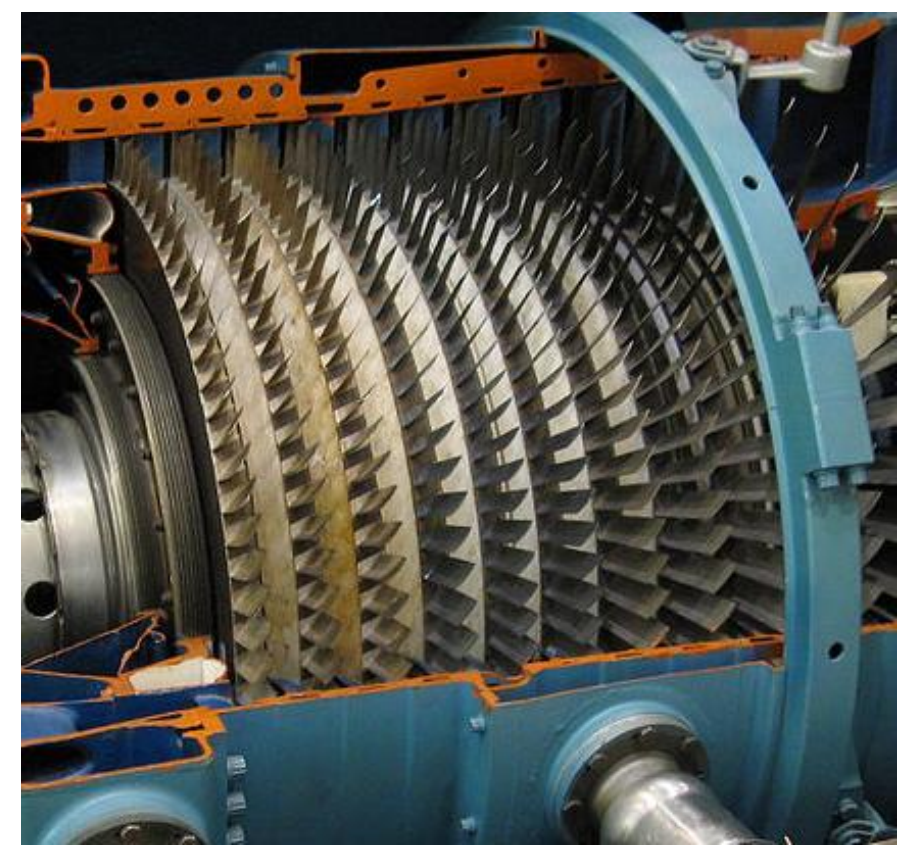

(a) Several rows in an axial flow compressor.

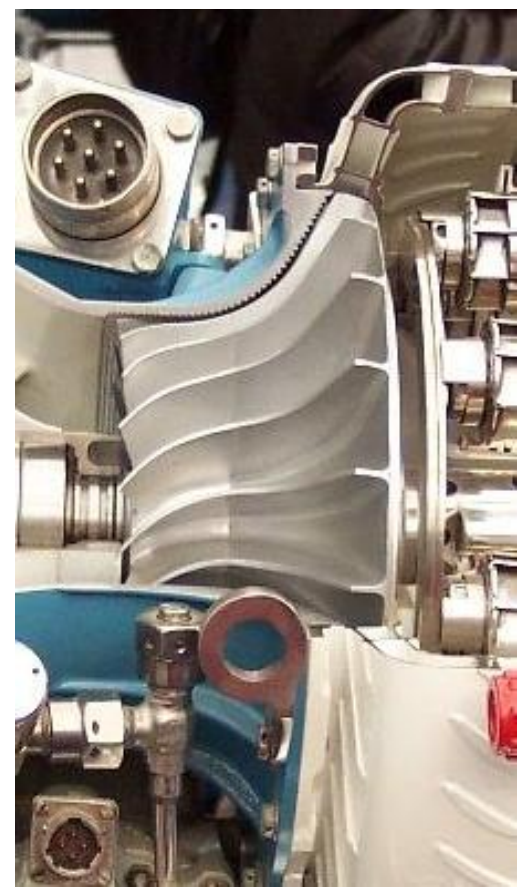

(b) Centrifugal flow compressor.

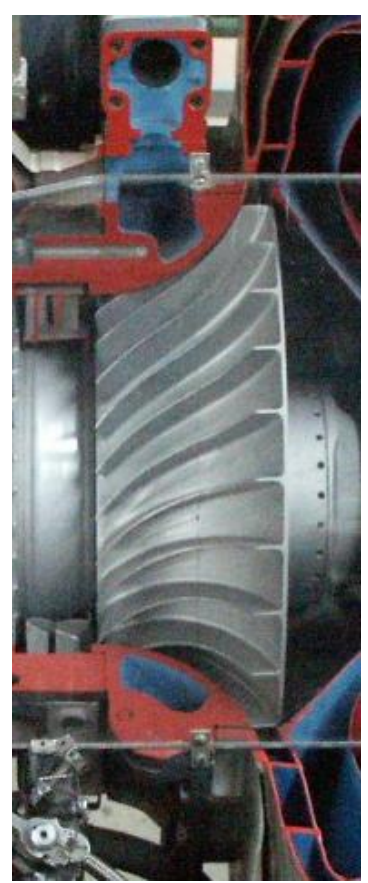

(c) Mixed-flow comp.

Figure 1.1 Various types of compressors [4] in (a) a TF30-P-6 Pratt \& Whitney engine, (b) a Lycoming T53 turboshaft engine, and (c) a Saturn MD-120 engine. 


\subsection{Instabilities in Axial Compressors}

Unfortunately, turbo-compressors also have shortcomings. Namely, that when a compressor approaches its limits of operational stability, unsteady aerodynamic instabilities arise from within the machine. If these instabilities are not treated and are allowed to grow, they may turn into either compressor stall or compressor surge. The characteristic of compressor surge is flow reversal, while compressor stall is characterized by the formation of low-momentum flow regions (cells) that rotate at a fraction of the rotor speed and obstruct the incoming flow through the duct. Such phenomena can have detrimental effects on the rest of the components of the compressor and the propulsion system. A lot of study has been done in order to predict the onset of such instabilities. In that regard, the analytical work of authors such as Greitzer [5-8], F.K. Moore [9], and R. D. Moore [10], has served as a basis for further investigation of flow-field conditions that lead to unstable flow phenomena. Furthermore, allowing a compression system to function at an operating condition where flow instabilities have a noticeable presence has been identified as a concern for high cycle fatigue. An example of this is a more recent work by Hah [11]. Hence, various methodologies have been devised in order to preserve or regain flow-field stability [12].

Enhancing the stability of compression systems is the field of interest of this work. Stability is considered to be the ability of a compressor to recover from disturbances that take a compression system away from an operational equilibrium point [13]. In this work, stability is also considered as the ability of a compression system to withstand the turbo-compressor's self initiated instabilities under adverse flow conditions.

Instabilities in the flow-field arise from interaction of secondary flows and other flow phenomena (e.g. passage shock) in the compressor. The secondary flow that has the most influence 
in the turbo-machine used in this work is the tip leakage flow $[14,15]$. This flow is driven from the pressure side to the suction side of a rotor blade by the pressure difference existing across the gap between the blade tip and the casing. The flow phenomena observed in axial flow compressors that leads to an unstable flow-field is the formation of end-wall blockage near the casing and the hub caused by boundary layer growth on these surfaces. Moreover, if the turbo-machine operates in the transonic regime, shock structures in the flow passage interact with the tip leakage flow to increase the chance for the formation of instabilities $[14,15]$.

\subsection{Stability Control Methods}

Various methods have been devised to enhance or recover stability in compressors. Based on whether or not flow is introduced into or bled off the duct flow, the stability method can be classified as passive or active.

\subsubsection{Active}

Active stability control on compression systems are brought by the action of devices that inject fluid into and/or bleed fluid out of the flow-field. Studies such as that by Niazi et al. [16] apply bleeding in regions of reversed flow, downstream of the rotor blades in an axial flow compressor. Their work showed that bleeding only $3 \%$ of the total mass flow enabled the extension of the operating range of the compressor. Work by Hathaway [17] shows that locating the bleeding or injection valves in strategic places of the casing can have a greater positive impact on range extension. Similar application of active devices on the flow-field of centrifugal compressors are presented by Stein et al. [18] and Skoch [19]. 


\subsubsection{Passive}

Passive methods consist of physical modifications of the components of the compressor in order to enhance stability. Therefore, there are no devices actively bleeding or injecting flow into the duct. Passive methods involve "casing treatment," which means making geometric modifications to the casing [12].

Among the literature surveyed is the work done by Takata et al. [20] They report using five forms of casing treatment, of which two variations of skewed slots are the most successful at increasing stall margin. The mechanism introduced by these casing treatments consists of fluid from low momentum regions near the blade tip being driven upstream of the blade and into the main flow region through the slots. Similar forms of casing treatment were used by Yu et al. [21] and Emmrich et al. [22,23]. A common finding of their work was that, due to a redistribution of the flow throughout the duct, the location where stall occurred changed from near the casing to a location near the hub. It was also observed that using the slots casing treatment delayed compressor stall until incidence angles attained large values.

Another form of casing treatment used in the experiments of Takata was circumferential grooves. Using them gave nearly the same total pressure rise as the smooth casing and had marginal impact on stall range extension. However, computational studies by Lin et al. [24,25] show that range extension by circumferential grooves is possible when their number and location along the blade tip is chosen strategically.

The type of casing treatment used in this work is that of studs (small solid structure) protruding off the casing treatment near the trailing edge of the rotor blades. A similar form of casing treatment has not been found in the literature. However, simulations performed in this work 
have shown potential for extension of the operating range. Details of the protruding studs will be provided in Chapter 4.

\subsection{CFD Application to Turbo-Machinery}

Computational Fluid Dynamics (CFD) has become an important tool in the study and design of turbo-machinery flow. It complements experiments by providing insight into the details of the flow that would otherwise be difficult to observe or measure using experimental methods. CFD consists of numerically solving the governing equations of fluid flow and enforcing the boundary conditions that characterize flow-fields in turbo-machinery. The present work and its findings make extensive use of CFD tools. The flow solver software used here is Tenasi, which was developed in-house. Further details about the software and the computational procedure are provided in Chapter 5. 


\section{CHAPTER 2}

\section{MATHEMATICAL DESCRIPTION OF THE FLOW}

In order to solve engineering problems, it is necessary to relate fluid properties and behavior in a formal and deterministic way. The purpose here is to be able to quantify property changes, intuit the flow behavior, and predict interactions within the fluid itself and with solid bodies.

The formality of the above mentioned relations is brought by algebraic, differential and integral equations. The deterministic characteristic of these equations is achieved by deriving the relations from the laws of mechanics that apply to fluid flow. Such laws are described for a system, but in order for the equations to be useful tools in fluid dynamics analysis they are derived using the concept of control volume.

\subsection{Control Volume vs. System}

A control volume can be imagined using Figure 2.1. 


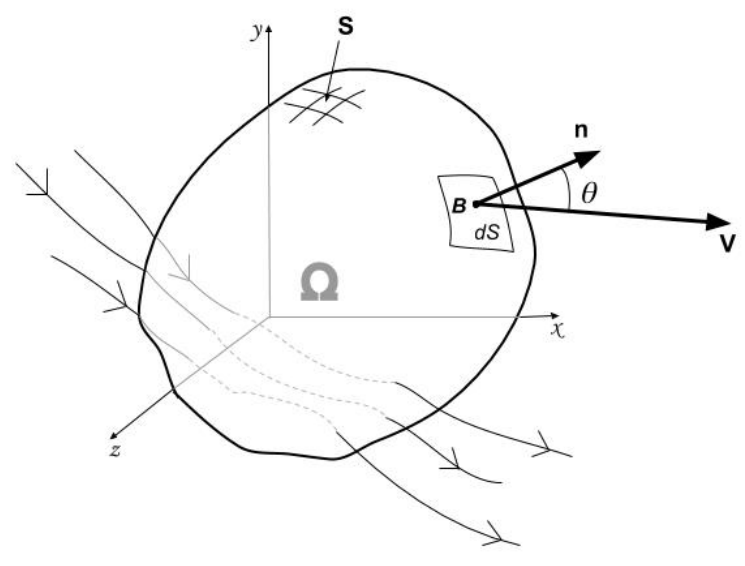

Figure 2.1 Depiction of a control volume.

A control volume is a region of space defined by surface called control surface. For the purpose of this text the control volume is labeled by $\Omega$, and the control surface is labeled by $S$. If a point $B$ is located on the control surface $S$, a differential area $d S$ of elemental size can be assigned to $B$. Also, a unit vector, $\mathbf{n}$, normal to the differential area $d S$, and pointing outward of the control volume, is associated to $d S$ at $B$, and $d \mathbf{S}=\mathbf{n} d S$ can be defined. Lastly, it can be conceived that the control surface $S$ is comprised of many of these elemental size differential areas $d S$, and that, similarly, the control volume $\Omega$ is comprised of many elemental size differential volumes $d \Omega$.

A system is a defined amount of mass chosen for study. Anything outside the system is called surroundings. Dividing the system from its surroundings is the system's boundary which 
can be real or imaginary, and fixed or movable. It is through the boundary that a system interacts with its surroundings.

The use of control volumes is preferred in aerodynamics for their inherent nature of allowing mass flow through the control surface. However, since quantifying changes of the thermodynamic properties of the fluid is also very important in fluid dynamics, it is helpful to observe how control volumes and systems relate to each other. Figure 2.2, which depicts a nearly one dimensional flow $V=V(x)$ through a duct, is presented for that purpose. It shows at time $t$ that the control volume between sections $a$ and $b$ is completely filled by system 2 . Then, it shows that at time $t+\delta t$ system 2 has partially moved outside of the control volume between a and $b$, and that the region of space abandoned by system 2 has been filled by part of system 1 . In this example, the take away is that if there are flow properties changing within the control volume between a and $b$, some of these changes can be attributed to the moving systems carrying fluid properties. 


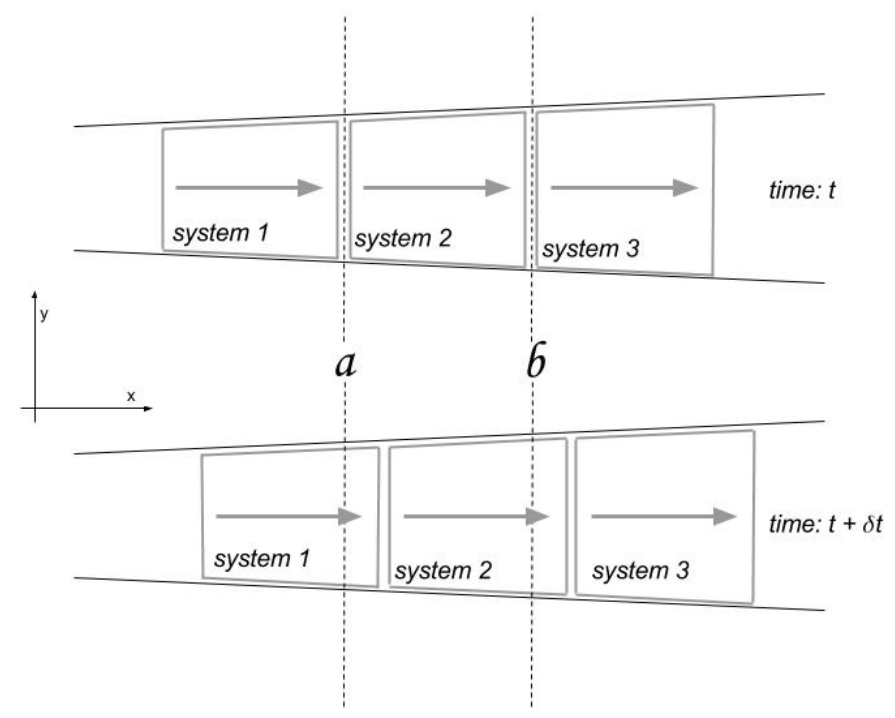

Figure 2.2 Relation between systems and control volumes [1].

\subsection{Equations Describing the Fluid Flow}

The governing equations are now derived from the physical laws.

\subsubsection{Conservation of Mass}

The conservation of mass principle states that within a system closed to any transfer of matter and energy, the amount of mass of the system remains constant over time. The meaning of this, in the context of defining a conservation of mass relation for a control volume, is that there is no such thing as a source or "sink" of mass to be taken into account. The rate of change (any variation in time) of mass inside the control volume would be attributed to the net mass flow through the control surface. This balance is shown in equation 2.1. 


$$
\frac{\partial}{\partial t} \int_{\Omega} \rho d \Omega+\oint_{S} \rho(\mathbf{V} \cdot \mathbf{n}) d S=0
$$

The first term on the left side of the equation is the time rate of change of the mass inside the entire control volume, where $\rho d \Omega$ is the mass contained in an elemental volume. In the second term, $\rho(\mathbf{V} \cdot \mathbf{n})$ represents a mass flux (mass per unit area per unit time). Therefore, the second term represents the mass flow through the entire control surface. The conservation of mass equation is also known as the continuity equation.

\subsubsection{Conservation of Momentum}

The conservation of momentum law, or Newton's second law of motion, says that the net force acting upon a body is equivalent to the body's time rate of change of momentum. Usually this statement is presented through the vector form equality $\mathbf{F}=m \mathbf{a}$, and $\mathbf{F}$ is the force $\mathbf{F}=d \mathbf{P} / d t$ where $\mathbf{P}$ is the momentum vector. For a control volume, however, the conservation of momentum principle is written as shown in equation 2.2.

$$
\frac{\partial}{\partial t} \int_{\Omega} \rho \mathbf{V} d \Omega+\oint_{S} \rho \mathbf{V}(\mathbf{V} \cdot \mathbf{n}) d S=\int_{\Omega} \rho \mathbf{f} d \Omega-\oint_{S} p \mathbf{n} d S+\oint_{S}(\overline{\bar{\tau}} \cdot \mathbf{n}) d S
$$

In the first term on the left hand side, $\rho \mathbf{V} d \Omega$ represents the momentum associated with a mass in an elemental volume and the integral is the sum of the momentum throughout the entire control volume. Therefore, the first term represents the changing momentum within a control volume of an unsteady flow passing through. The second term is the sum over the entire control surface of the momentum flux, momentum carried by fluid mass passing through an elemental 
surface $d S$. On the right hand side, within the first term is $\mathbf{f}$ which represents body forces per unit mass in an elemental volume. Such body forces can be produced by gravity, electromagnetic fields, spin forces, or other fields that act on the volume of matter. Therefore, the first term is a volume integral of the body forces acting on the mass contained in the control volume. The second and third terms are surface integrals of forces produced by pressure and shear stresses, respectively, acting on elemental surfaces over the entire control surface. The second term has a negative sign because the forces resulting from pressure, $p$, point inward on the control surface. In the third term, $\tau$ is the shear stress tensor.

\subsubsection{Conservation of Energy}

As specified for a closed system, the first law of thermodynamics states that the change of internal energy of a system is equivalent to the amount of heat transferred into the system minus the amount of work done by the system. In the case of flow through a control volume, the kinetic energy of the fluid should also be considered along with its internal energy. The conservation of energy relation for a control volume (assuming no appreciable change in potential energy) is presented in equation 2.3.

$\frac{\partial}{\partial t} \int_{\Omega} \rho E d \Omega+\oint_{S} \rho E(\mathbf{V} \cdot \mathbf{n}) d S=\int_{\Omega} \rho(\mathbf{f} \cdot \mathbf{V}+\dot{q}) d \Omega-\oint_{S} p(\mathbf{V} \cdot \mathbf{n}) d S+\oint_{S}(\overline{\bar{\tau}} \cdot \mathbf{V}) \cdot \mathbf{n} d S+\oint_{S} k(\nabla T \cdot \mathbf{n}) d S$

The quantity $E$ on the left hand side is the sum of the internal energy $e$ per unit mass and the

kinetic energy $|V|^{2} / 2$ per unit mass of the fluid within the control volume. Then, as for the previous 
two conservation equations, the first term on the left hand side represents the rate of change of the total energy within the control volume; and the second term represents the net flow of total energy, where $\rho E(\mathbf{V} \cdot \mathbf{n})$ is called the energy flux.

On the right hand side, the first term accounts for both the work done by the body forces and the volumetric heating of the fluid within the control volume. The second and third terms account for the work resulting from forces acting on the surface of the control volume, pressure and shear stress, as in the momentum equation. Finally, the last term is a diffusive term that takes into account heat flux driven by temperature gradients. Fourier's law of heat conduction is used to quantify heat flux as: $\mathbf{F}_{D}=-k \nabla T$, where $k$ is the thermal conductivity coefficient.

\subsubsection{Thermodynamic Relations for Perfect Gas}

In order to solve the system for all the variables necessary to define the flow, two more equations are necessary. Assuming that air behaves as a calorically perfect gas, the equation of state is used:

$$
p=\rho R T
$$

and the enthalpy is obtained from:

$$
h=c_{p} T
$$


Using the following definitions:

$$
\begin{array}{r}
R=c_{p}-c_{v} \\
\gamma=\frac{c_{p}}{c_{v}}
\end{array}
$$

The equation of state can be recast into:

$$
p=(\gamma-1) \rho\left[E-\frac{u^{2}+v^{2}+w^{2}}{2}\right]
$$

\subsubsection{Complete System in Vector Form}

The following is the set of governing equations written in vector form. Recasting the equations this way allows for full appreciation of all the terms involved in the system.

$$
\frac{\partial}{\partial t} \int_{\Omega} \mathbf{W} d \Omega+\oint_{S}\left(\mathbf{F}_{c}-\mathbf{F}_{d}\right) d S=\int_{\Omega} \mathbf{Q} d \Omega
$$

The vector $W$ contains the conserved variables. Vectors $\mathbf{F}_{c}$ and $\mathbf{F}_{d}$ contain the convective and diffusive fluxes, respectively. Vector $Q$ contains volume sources due to heat generation and body forces.

$$
W=\left(\begin{array}{c}
\rho \\
\rho u \\
\rho v \\
\rho w \\
\rho E
\end{array}\right)
$$




$$
\mathbf{F}_{c}=\left(\begin{array}{c}
\rho V \\
\rho u V \\
\rho v V \\
\rho w V \\
\rho H V
\end{array}\right) ; \quad V=\mathbf{V} \cdot \mathbf{n}=n_{x} u+n_{y} v+n_{z} w
$$

$$
\mathbf{F}_{d}=\left(\begin{array}{c}
0 \\
n_{x} \tau_{x x}+n_{y} \tau_{x y}+n_{z} \tau_{x z} \\
n_{x} \tau_{y x}+n_{y} \tau_{y y}+n_{z} \tau_{y z} \\
n_{x} \tau_{z x}+n_{y} \tau_{z y}+n_{z} \tau_{z z} \\
n_{x} \Theta_{x}+n_{y} \Theta_{y}+n_{z} \Theta_{z}
\end{array}\right) \quad ; \quad \Theta_{y}=u \tau_{y x}+v \tau_{y y}+w \tau_{y z}+k \frac{\partial T}{\partial y}
$$

$$
\mathbf{Q}=\left(\begin{array}{c}
0 \\
\rho f_{x} \\
\rho f_{y} \\
\rho f_{z} \\
\rho \mathbf{f} \cdot \mathbf{V}+\dot{q}
\end{array}\right)
$$




\section{CHAPTER 3}

\section{COMPRESSION SYSTEM AND CASING TREATMENT}

In the present section, a description is given of the casing treatment and the compression system used for testing. Some insight on the origin and data available on both equipment is given. References are provided if further details are desired.

\subsection{SDT2-R4}

\subsubsection{Overview}

The test compression system used for the casing treatment under study is the SDT2-R4. It is an axial flow transonic turbofan stage for which a model was available and aerodynamic performance data has been published by Hughes [2]. It has previously been used at the SimCenter for validation of the in-house flow solver Tenasi, as well as for testing solver features that facilitate turbomachinery simulations $[26,27]$. Other casing treatment studies have also been performed using this turbofan $[24,25]$. Figure 3.1 shows the SDT2-R4 model installed at the NASA Glenn $9 \times 5$ LSWT [28]. 


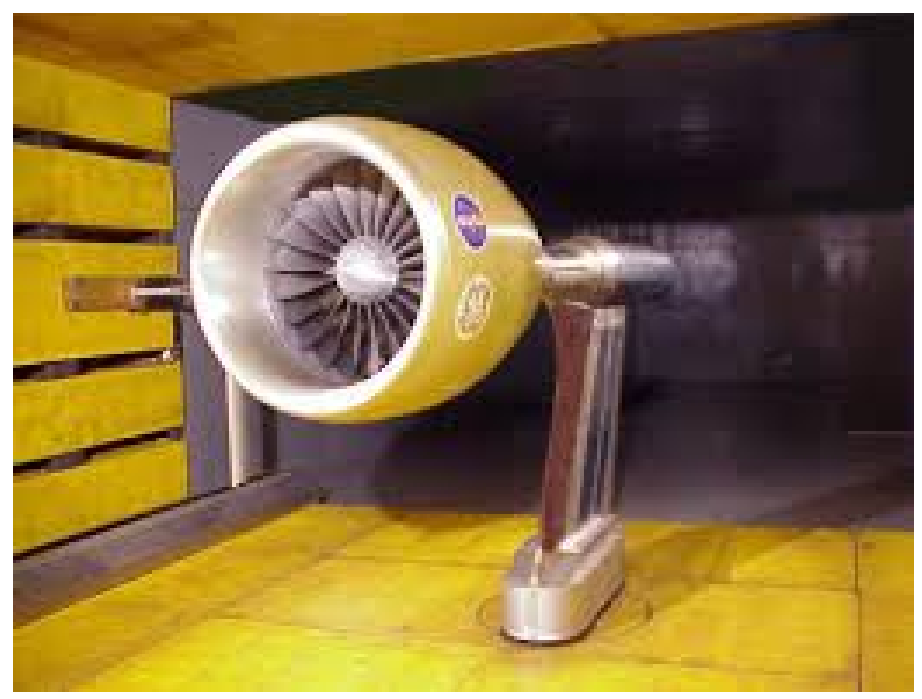

Figure 3.1 SDT2-R4 installed in wind tunnel.

The SDT2-R4 is a $20 \%$ scale model that represesents the bypass stage of a high bypass ratio turbofan stage, representative of the technology used at the time of its creation in the early 2000's. It was developed by General Electric Aircraft Engines (GEAE) as part of a cooperative effort with NASA on a program called "The Source Diagnostic Test" (SDT). The program had the following objectives: to establish the performance of the fan model used, the GEAE developed R4; to assess the performance of the fan when coupled with three different outlet guide vane (OGV) designs; to determine the OGV's effect on the fan performance; and to conduct detailed acoustic and aerodynamic diagnostic surveys in order to understand the noise generation mechanisms in a turbofan engine [2]. In the SDT program, only the bypass section and not the power core section was considered [2]. Furthermore, only the Baseline OGV from the SDT program is used in the current work. Table 3.1 presents a summary of the design parameters of the SDT2-R4. 
Table 3.1 Stage design parameters

\begin{tabular}{|ll|}
\hline No. of Fan Blades & 22 \\
Fan Tip Diameter & 22 in \\
Corrected RPM & 12,657 \\
Corrected Tip Speed & $1,215 \mathrm{ft} / \mathrm{sec}$ \\
No. of Stator Vanes & 54 \\
Corrected Weight Flow & $100.5 \mathrm{lbm} / \mathrm{sec}-\mathrm{ft}$ \\
Stage Pressure Ratio & 1.47 \\
\hline
\end{tabular}

\subsubsection{Performance Considerations}

The plots in Figure 3.2 are experimental values of total pressure ratio and the adiabatic efficiency of the stage at hand. According to Hughes [2], in the experimental procedure, a Variable area Fan Exit Nozzle (VFEN) was used to obtain the performance curves below for a range of fan speed operating conditions. Through the VFEN, the exit area varied, in turn changing the back pressure on the fan to test different operating points that resembled changes in aircraft flight speed and altitude.

An important consideration from the SDT2-R4 performance curves available is that the left most points are not representative of stall conditions. It is usually the case, that the most right operating point in a performance curve represents the choking condition (where a higher mass flow through the duct is physically not possible), and that the most left operating point represents the stall condition (unstable flowfield at low mass flow rate). However, Hughes reports that the stall condition was avoided in order to avoid the risk of damaging the fan blades. When testing the fan alone, the approaching stall condition was determined by an increase in the fan blade stress being measured by blade mounted strain gauges. Furthermore, the lowest mass flow rate point tested for the entire stage was set higher than that obtained for the fan alone because, at that point, the strain 
gauges had stopped working. This fact is important and will be taken into consideration later when reporting the results of the simulations.

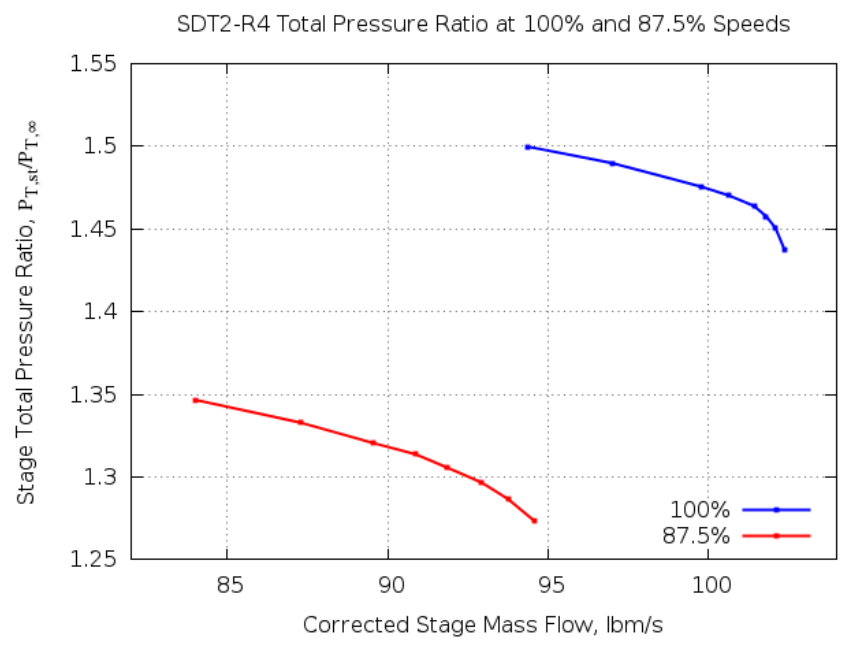

(a) Total Pressure Ratio

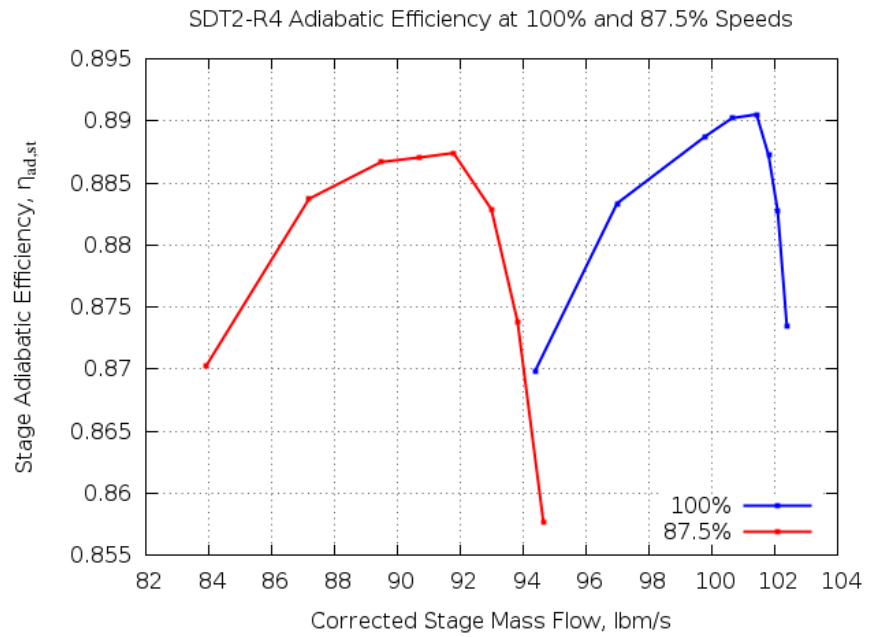

(b) Adiabatic Efficiency

Figure 3.2 Experimentally measured performance curves for the SDT2-R4 stage [2]. 


\subsubsection{Modification}

One important modification on the model of the SDT2-R4 is the addition of one stator vane. Previous computational studies have been performed on this stage with the added stator vane [26,27], which concluded that this addition does not significantly impact the performance of the stage. The importance of adding an extra stator vane is that now the model lends itself for simulation of a one-eleventh slice of the duct (two fan blades and five stator vanes), instead of a half or the full wheel, in turn saving on computational resources while carrying out this work. Figure 3.3 depicts the full wheel of the SDT2-R4 with the added stator vane.

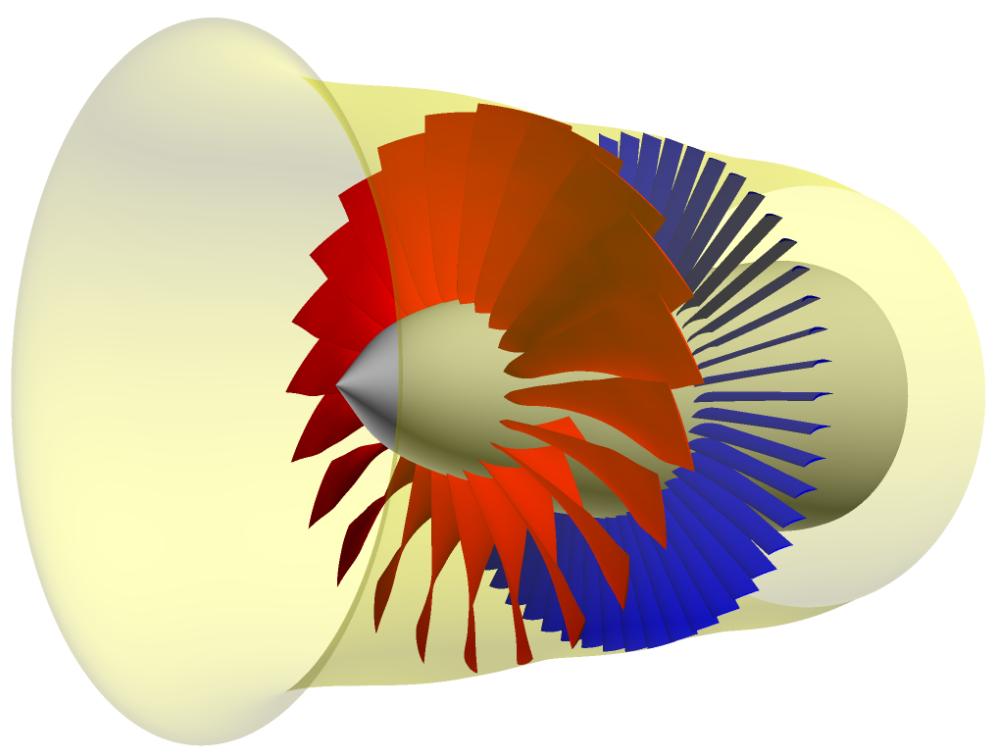

Figure 3.3 Full wheel of simulated stage.

\subsection{Protruding Studs}

The casing treatment used in this work is protruding studs. From survey of the literature, it is realized that using protruding studs has not been explored as a form of casing treatment. This 
section provides a description of the protruding studs and how they came to be considered as a potential form of casing treatment.

\subsubsection{Description}

The studs are solid structures protruding from the casing into the duct. They are located downstream of the fan blades and in the vicinity of their tip. Two shapes of studs have been tested, elongated "U's" and dome shaped studs. The description of each is provided next. Variation in dimensions of the "U" shaped studs were tested, while a single configuration of the dome shaped studs was simulated.

\subsubsection{Elongated “U” Shape}

The cross section in the tangential direction (with respect to the wheel) of these type of studs have the shape of a "U." These were the first type of studs tested. The first configuration used extended in the tangential direction an angle of a fifth of a rotor pitch or approximately $3.27^{\circ}$. The thickness and depth of protrusion are expressed in relation to the fan blade chord length at the tip, and they are approximately $3.2 \%$ (or $0.11 \mathrm{in}$ ) and $4.5 \%$ (or $0.16 \mathrm{in}$ ), respectively. Their location from the trailing edge at the tip is about $2.9 \%$ (or 0.10in). The sides of the original studs were aligned with the stagger of the fan blades at the tip. The number of these type of studs used is 55, and they are equally spaced. Because this was the first configuration of studs tested, they will be referred to by the term "S1" through the rest of this manuscript. Soon after the results of the simulations with the S1 configuration was available, a second configuration of the studs were conceived, where the sides are aligned with an angle opposite to the fan blade stagger at the tip. The second configuration of studs receives the code "S2" through the rest of the manuscript. Figure 3.4 shows the actual 
models on the casing, and Figure 3.5 is a graphical description of the dimension of studs S1 and S2.

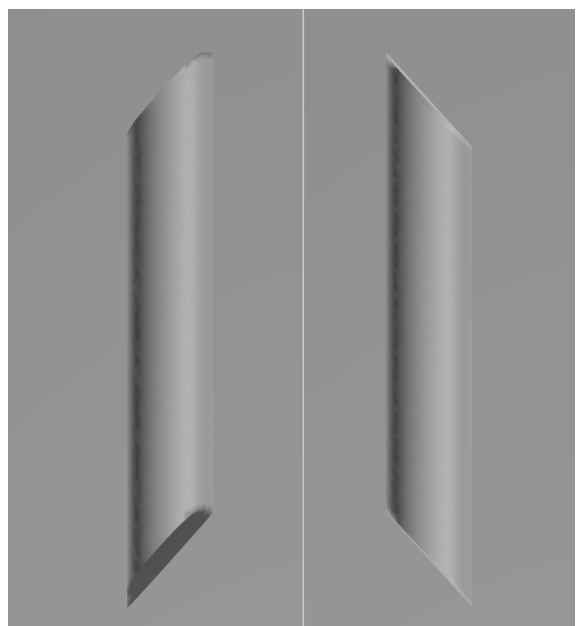

(a) From right to left: $\mathrm{S} 1$ and $\mathrm{S} 2$

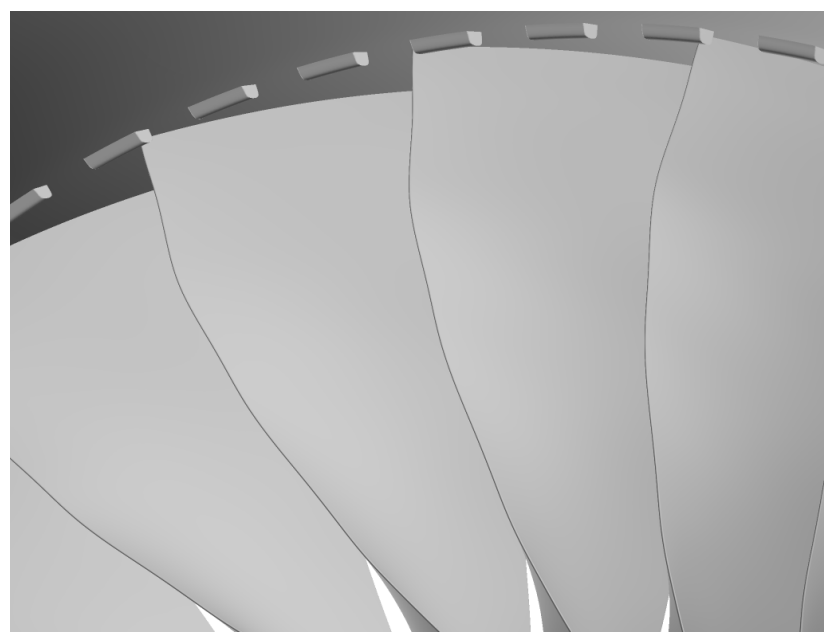

(b) Aft-looking-forward view of studs and blades.

Figure 3.4 Protruding studs S1 and S2

Testing of these configurations showed an improvement in the Stall margin [29] (details in Chapter 6). Based on these results, it was decided to test variations of These designs. Variations tested included half depth studs (50\% of original, H1 \& H2), $125 \%$ of original(L1 \& L2), and $150 \%$ of original depth (X1 \& X2). Finally, the last "U" shaped type of studs to be tested were similar to the S2 studs but were to extend tangentially only $50 \%$ of the original, and twice as many of them (110) were to be used, equally spaced and in the same location with respect to the fan blades (W50). 


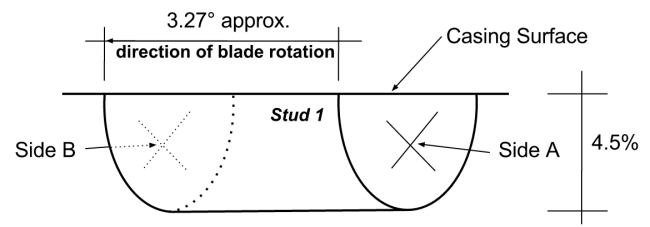

(a) Front view: tangengial extension and depth of protrusion.

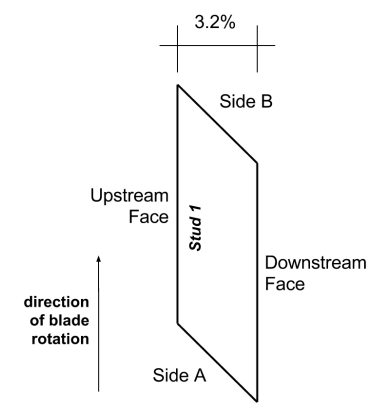

(b) Top view: width.

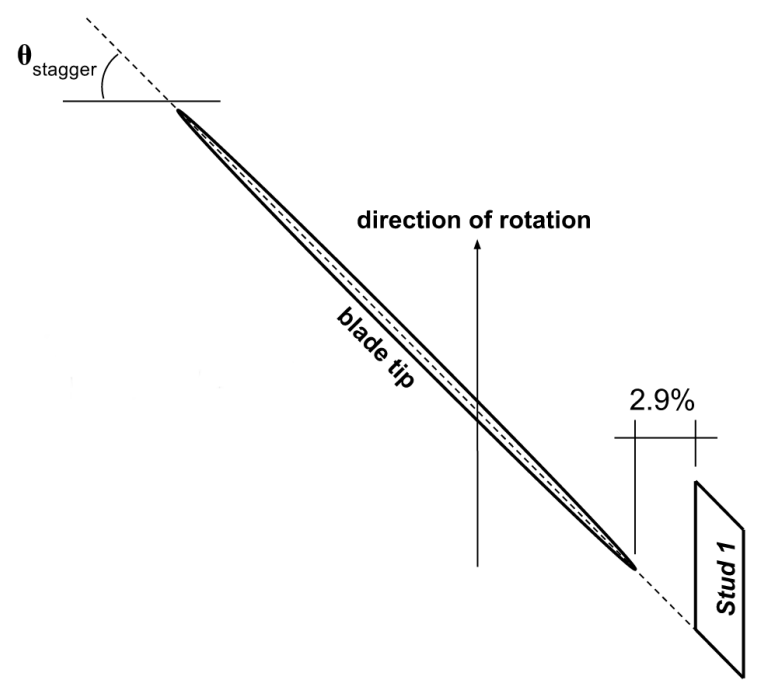

(c) Top view: separation from trailing edge and alignment of sides in Studs 1.

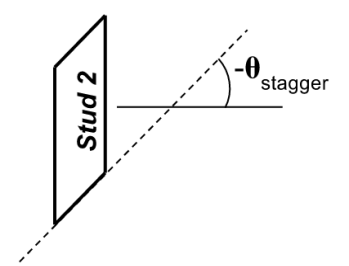

(d) Top view: Alignment of sides in Studs 2.

Figure 3.5 Dimensions as percentage of fan chord length at tip, alignment, and location of studs. 


\subsubsection{Dome Shape}

The dimensions of the studs, as with those presented in the previous section, can be manipulated in many ways, and different shapes of studs could be tested until finding a design that has the greatest positive impact on range extension without having a detrimental effect on efficiency. In order to move in that direction, one more shape of studs was explored. This stud is formed by a half revolution of the "U" shape about a vertical axis at the middle of this shape. The resulting surface of revolution resembles a dome protruding out of the casing. This type of studs receive the code " $\mathrm{D}$ " for the rest of this manuscript. The diameter and depth of protrusion of the D studs in terms of the fan blade chord at the tip are approximately $3.2 \%$ (or $0.11 \mathrm{in}$ ) and $4.5 \%$ (or 0.16in). Their location behind the trailing edges of the fan blades at the tip is approximately $2.9 \%$ (or $0.10 \mathrm{in}$ ), and there are 220 of them equally spaced across the casing. Figure 3.6 shows the domes downstream of the fan blades.

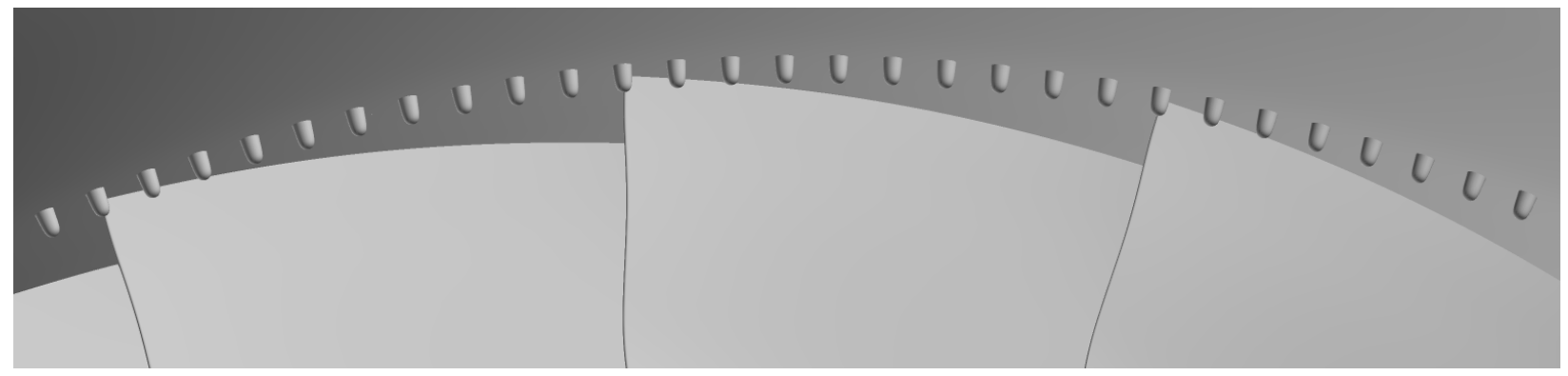

Figure 3.6 Aft-looking-forward view of dome studs and blades.

\subsubsection{Origin}

In his master's thesis research, Lin worked with the same piece of turbomachinery and examined two forms of casing treatment [3]. One consisted of rings protruding into the flowfield, 
located near the leading edges and the trailing edges of the fan blades. He tested three combinations of the rings: both together, a single one near the leading edge, and a single one near the trailing edge, as shown in Figure 3.7.

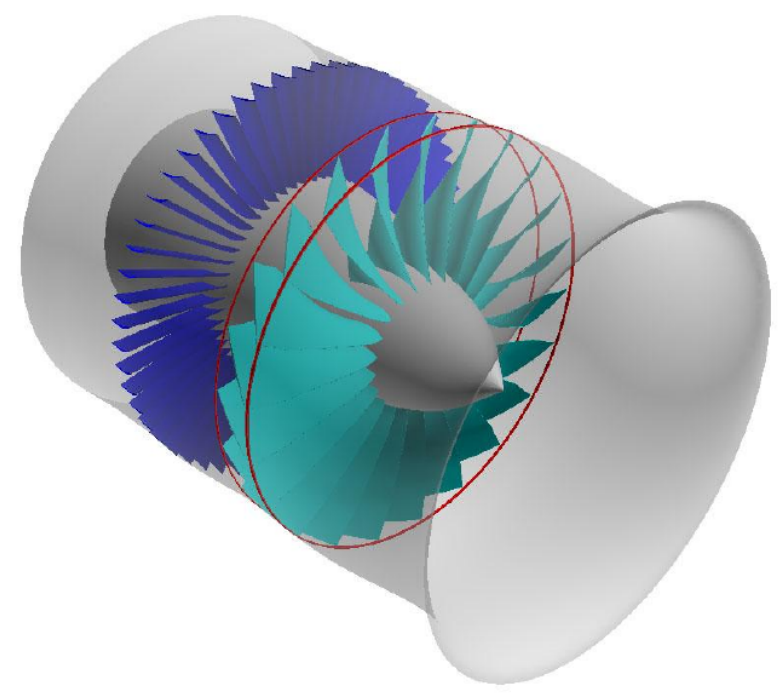

(a) Front and back ring locations in relation to fan stage

(a) Model showing both rings.

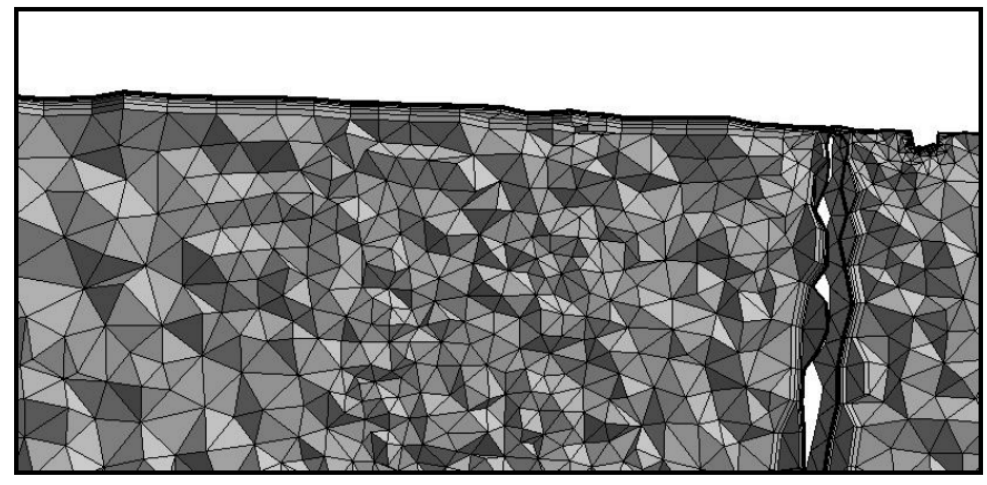

Figure 3.11 A theta cut of the grid for trailing-edge ring only

(b) Volume mesh with gap for trailing edge ring.

Figure 3.7 Protruding rings from Lin's work [3] (including caption from original text). 
As Lin reports, the first two configurations had detrimental effects on the performance of the turbofan. Using the single one near the trailing edge, however, had an insignificant change in total pressure ratio with a small reduction in adiabatic efficiency compared to the baseline performance of the turbofan, as simulated. The performance curves from his work are shown in Figure 3.8. The idea occurred, then, to modify the ring by cutting sections from it to let some fluid through, and see what the behavior of the flow would be. Although it went undocumented, a simulation was carried out which a relative increase in range with a small decrease in efficiency. Hence the source for the idea of the, now called, protruding studs in this study. The dimensions and location of the trailing edge ring in Lin's work were carried directly into this work.

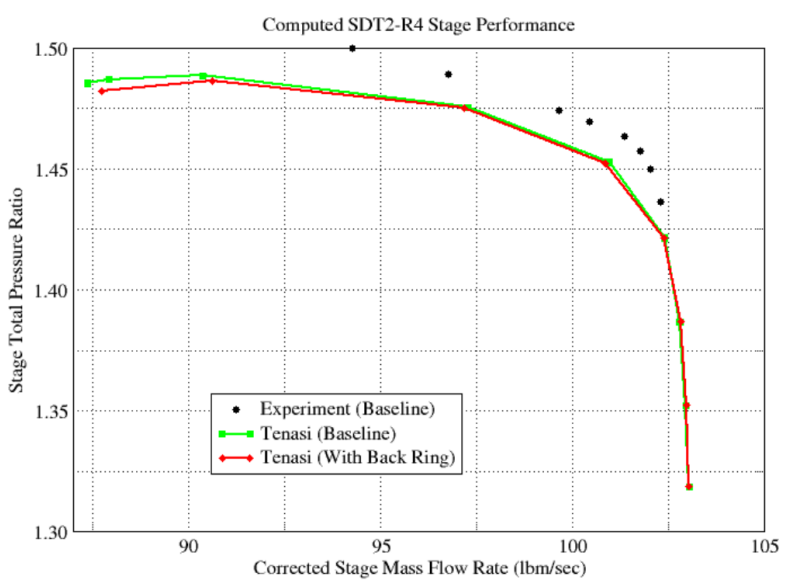

(a)

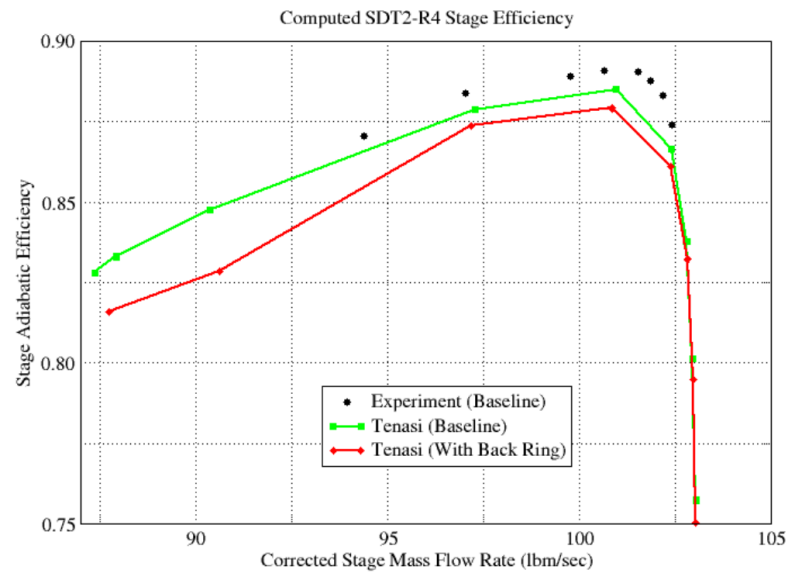

(b)

Figure 3.8 Performance curve using trailing edge ring [3] 


\subsubsection{Search for Similar Form of Casing Treatment}

After doing a survey of current literature, no report on the deliberate use of a similar type of casing treatment as the one under study here has been found. A similar effect from a stability control system was found in a piece of work by Skoch [19] where he reports the effects of introducing bleed or control tubes into the flowfield of a centrifugal compressor, from the shroud and downstream of the impeller blades in the diffuser section. An unexpected finding from his experiments was that the mere presence of the control tubes had an stabilizing effect on the flowfield without executing their function of bleeding fluid. Skoch's report [19] reinforces the idea of possibly enhancing the stability of a flowfield by introducing structures in key regions of the duct in a compression system. 


\section{CHAPTER 4}

\section{COMPUTATIONAL PROCEDURE}

\subsection{Flow Solver}

This section introduces the software used for performing numerical simulations and exposes some important aspects of the computations it performs. Intricacies of the numerical schemes for time and space discretizations, solutions of linear systems, stability enhancement of linear solvers, turbulence modeling, etc. are bypassed in this text since this work is focused on the stability of the operation of compression systems. References are provided as such aspects are described for when further detail is desired.

\subsubsection{Tenasi}

The flow solver used through the computational study reported in this manuscript is Tenasi. It has been developed at the SimCenter, at the University of Tennessee at Chattanooga, and it is used for solving a great variety of engineering problems that involve fluid flow. The range of such problems includes bronchial flows, plume dispersion modeling in urban environments, reacting flows, free surface modeling, pumps, compressors, etc.

Tenasi is a finite volume flow solver which uses an unstructured, node-centered, implicit numerical scheme. It can handle flow regimes from incompressible [30] to compressible [31]. Various options are available for turbulence modeling. Generalized interpolative interfaces and 
relative frame capabilities are implemented in Tenasi $[26,27]$, which allow for computing flow on rotating and stationary frames interacting through a sliding interface. The solver software can run on single processor machines or on a parallel cluster of computers when solving large problems is necessary.

\subsubsection{Aspects of Numerical Solution}

\subsubsection{Spatial Discretization}

Representation of the space domain is carried through the use of a computational mesh. A computational mesh consists of nodes that are interconnected to form 3D cells of basic shapes. Further details on computational meshes will be provided in section 4.2.1. The flow solver Tenasi takes advantage of the mesh by using it for the spatial discretization. Control volumes are defined around the grid nodes, for which Tenasi is said to be a node-based finite-volume flow solver. The control volumes are defined by median-duals created between the mesh cells centroids. Then, the dual-faces are used as elements of the control surface for flux calculation. If the control volume, as defined in this section, is assumed not to change in time, equation 2.8 can be written as follows:

$$
\frac{d \mathbf{W}}{d t}=-\frac{1}{\Omega}\left[\sum_{m=1}^{N_{F}}\left(\mathbf{F}_{c}-\mathbf{F}_{d}\right)_{m} \Delta S_{m}-(\mathbf{Q} \Omega)\right]
$$

In order to calculate the net flux on each control volume, the flux contributions across the faces should be established. The inviscid fluxes are approximated by solving a one dimensional approximate Riemann problem for information provided at both sides of the face, a method called the Roe scheme [32]. The viscous fluxes are approximated using the directional derivative method, 
which combines information from solution gradients at the connectors (lines connecting two adjacent points) and information available through the connectors to approximate the gradients required by the viscous terms.

\subsubsection{Temporal Discretization}

The next step is the approximation for the time derivative term in the governing equations. Equation 4.2 shows a compact recast of equation 4.1 .

$$
\Omega \frac{d \mathbf{W}}{d t}=-\mathbf{R}
$$

The following non-linear scheme is an approximation of the time derivative [32]:

$$
\frac{\Omega \Delta \mathbf{W}^{n}}{\Delta t}=-\frac{\beta}{1+\omega} \mathbf{R}^{n+1}-\frac{1-\beta}{1+\omega} \mathbf{R}^{n}+\frac{\Omega \omega}{(1+\omega) \Delta t} \Delta \mathbf{W}^{n-1} \quad ; \quad \Delta \mathbf{W}^{n}=\mathbf{W}^{n+1}-\mathbf{W}^{n}
$$

In the equation above $n$ represents the current time-step level. If $\beta=0$, the time-stepping scheme is called explicit. A very basic explicit single-stage scheme is obtained by also setting $\omega=0$. Then, the time derivative is calculated using a forward difference, and the residual is calculated at the current time step [32].

If $\beta \neq 0$ in equation 4.3 , the scheme is called implicit, and its advantage over a explicit scheme is increased robustness while being able to use relatively larger time steps. It is a 2 nd order implicit scheme when $\beta$ and $\omega$ meet the condition that $\beta=1 / 2+\omega[32]$. 


\subsubsection{Turbulence Modelling}

A model is necessary to simulate turbulent flows with high Reynolds numbers. Performing a direct numerical simulation by the time-dependent governing equations is prohibitively expensive and can only be done for simpler problems at small Reynolds number values. In this work, Tenasi is set to use the one equation $k-\epsilon$ SAS turbulence model [33]. Such a model is incorporated in a "loosely-coupled" procedure, which means that the mean flow equations are first solved before solving the turbulence model independently [34].

\subsubsection{Boundary and Initial Conditions}

Boundary conditions are specified that characterize the problem as a duct flow and that truncate the physical space to a domain size that is manageable. The way they are handled is by applying the correct fluxes through the faces of the control volumes belonging to various boundaries [34]. The inflow boundary is set to desired for stagnation pressure and stagnation temperature, usually standard day total conditions. It is possible to specify the direction of the flow but not necessary. At the outflow, the value of static pressure, also called back pressure, is set to a value that will establish the conditions of the flow field between the choke point of the duct and compressor stall. The flux through both inflow and outflow boundaries is handled by a method called characteristic boundary conditions. At the solid surfaces, a adiabatic no-slip condition is enforced by applying the surface velocity on the flow and by making the velocity values at the surface nodes remain unchanged in the linear system. Axisymmetry conditions are set in the tangential direction in order to truncate the duct flow-field from a "wheel" to a "slice." By doing this, the size of the mesh is reduced and less computational resources are necessary. 
At these boundary conditions the values of the variables on one side of the slice are interpolated to the other side. Finally, generalized interpolative surfaces are established between two adjacent domains $[26,27]$. This feature is useful in order to transmit variables from one domain to the other even when one domain is rotating and the other is stationary.

The initial conditions set on the flow-field for each case in this work (i.e. baseline simulation, simulation with a configuration of studs) depend on the operating condition intended to be simulated. In order to compute a set of performance curves for the stage, a first case is run with initial velocity set to zero everywhere in the duct, with the rotational speed of the rotor set to a desired value, and with the outlet boundary condition set to a static pressure that (in the simulation) will cause the duct flow to choke. Once the computed mass flow through the duct converges to a certain value, the simulation is stopped and a new case is run. A new case is set up with a higher value of static pressure at the outlet boundary and the solutions from the case previously run (i.e. velocity, density, pressure.). Once, the simulation results show mass flow convergence, the simulation is stopped for the given back pressure and the process is repeated until the entire performance curve has been computed.

\subsection{Mesh Generation}

\subsubsection{General Concepts}

The flow-field and the solid surfaces that interact with the fluid are represented using points, also called nodes. It is at these points that the variables to be solved are stored, rates of changes are determined, volume and surface integrals of sources are evaluated, and convective and diffusive fluxes between points are calculated. A computational mesh or grid is created by interconnecting 
the points through connectors (or edges). Cells are formed with such connectors, which are shaped as triangles and quadrilaterals on surfaces, and tetrahedra, pyramids, prisms, and hexahedra in space. This process of subdividing the physical space through cells of basic shapes is called discretization of the space. The type of mesh used for discretization in this work is unstructured because indexing of the nodes follows no particular scheme, and a mix of different cell shapes is used.

Generation of a computational mesh is the first step in carrying out a simulation. As mentioned above, it is the way in which the flow-field for simulation is represented. Arguably, the mesh generation phase is also the most time consuming. Although, there exist simulation software packages with automatic mesh generation capabilities that can complete the task in little time and with minimum user interaction, a problem such as the one at hand requires dedicated attention to detail in different locations of the field along with a priori knowledge of the behavior of the flow. The tedious nature of this phase comes from the complexity of the geometry and the necessity to capture features of the flow which are at the heart of the problem. These challenges, and possibly having to recreate or modify the mesh if the solutions are not satisfactory, make for a process that requires a lot of user work and time.

The software used for the mesh generation work is Pointwise [35]. It is a mesh generation software package with capabilities for building structured and unstructured meshes. The tools featured in Pointwise are conducive for control of point distribution, creation of viscous layers on surfaces in volume meshes, and mesh optimization. In addition, it possesses CAD capabilities which are handy when complex surface meshing is required or no CAD geometry is provided. 


\subsubsection{Procedure}

The process of mesh generation starts with the definition of connectors. The connectors are laid along the edges of the domain with an optimal point spacing based on experience to capture the geometry and some expected flow features. They can be laid within surfaces, as well, if control of point spacing in regions of such surfaces is difficult. Some of the point spacings (in inches) used on the stage are shown in Figure 4.1. This gives an idea of the level of surface mesh refinement when considering that the fan rotor diameter is $22 \mathrm{in.}$

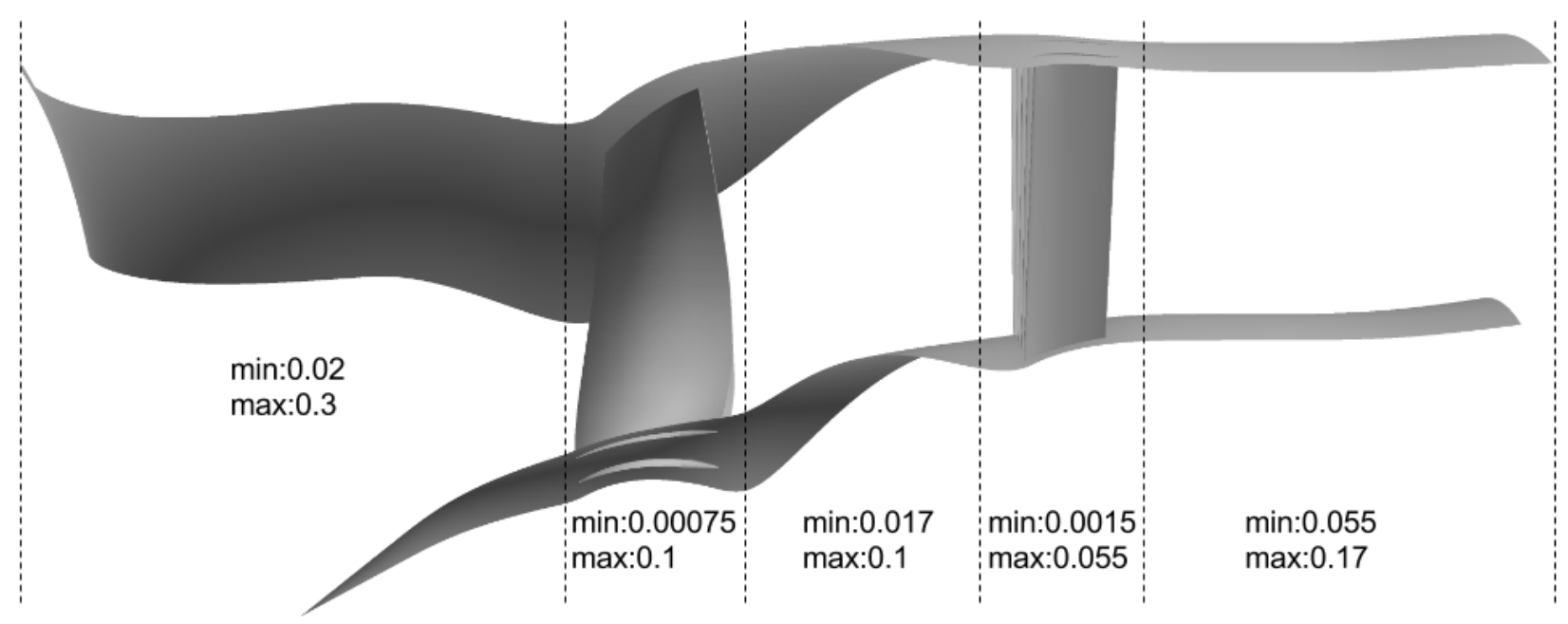

Figure 4.1 Minimum and maximum point spacing used in different regions of the turbofan stage

Following the definition of the edges is the creation of surface meshes. All domain boundaries are represented by surface meshes, whether solid or permeable. Since unstructured meshes are used in this work, the surface meshes are composed by triangles and are defined by a set of connectors. The mesh generation software provides control of the arrangement of points on surface meshes, and it also allows for gradual increase of point spacing in a certain direction away from 
the defining connectors. This is useful when creating viscous layers off solid surfaces. Figure 4.2 depicts the final surface mesh on the turbofan stage used for the majority of this work.

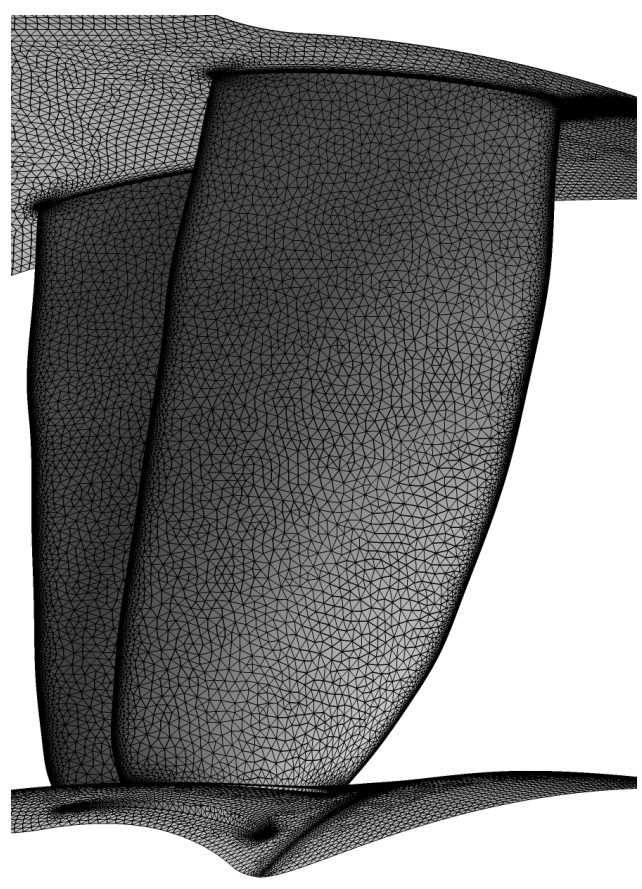

(a) Rotor blades

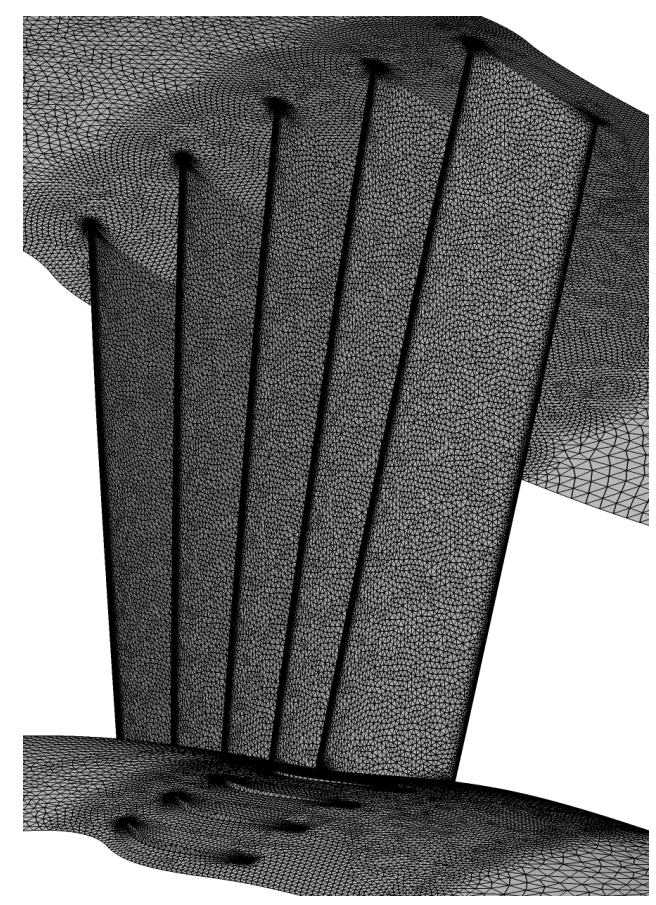

(b) Stator blades

Figure 4.2 Surface mesh on parts of stage

Next is the creation of volume meshes. There are three parts to this phase. The first phase is the definition of volume blocks enclosed by surface meshes, a process carried out manually. Secondly, the creation of viscous layers is included in the volume mesh to capture the viscous boundary layer. The mesh generation software possesses tools for this task. It requires provision of the spacing for the first viscous layer off the solid walls, a linear rate of growth for the subsequent layers, and the setting of a minimum and maximum number of layers to be included. The calculation 
of the first layer spacing off the solid walls is provided by viscous flow theory based on the concept of the non-dimensional wall distance $y^{+}$. Web based tools provided by universities and software companies are available to carry out these calculations seamlessly. For proper capturing of the boundary layer the value of $y^{+}$is set to 1 , and for a Reynolds number $10^{7}$ the computed wall spacing is approximately $4.5 \times 10^{-5}$ in which is set as the height of the first viscous layer in the mesh. A growth rate of 1.25 is chosen based on experience of previous work with turbo-machinery. A minimum number of 10 layers is enforced, and a maximum number of 16 layers is allowed based on testing reported later in Chapter 6. The last part in this process after the extrusion of viscous layers is filling out the rest of the block with tetrahedron shaped cells. The software automatically takes care of this task by inserting cells, the size of which gradually changes from the minimum to the maximum point spacings on the surfaces of the block. Figure 4.3 shows Pointwise visualizations of the viscous layer extrusion and a cut into the finalized volume mesh around the studs.

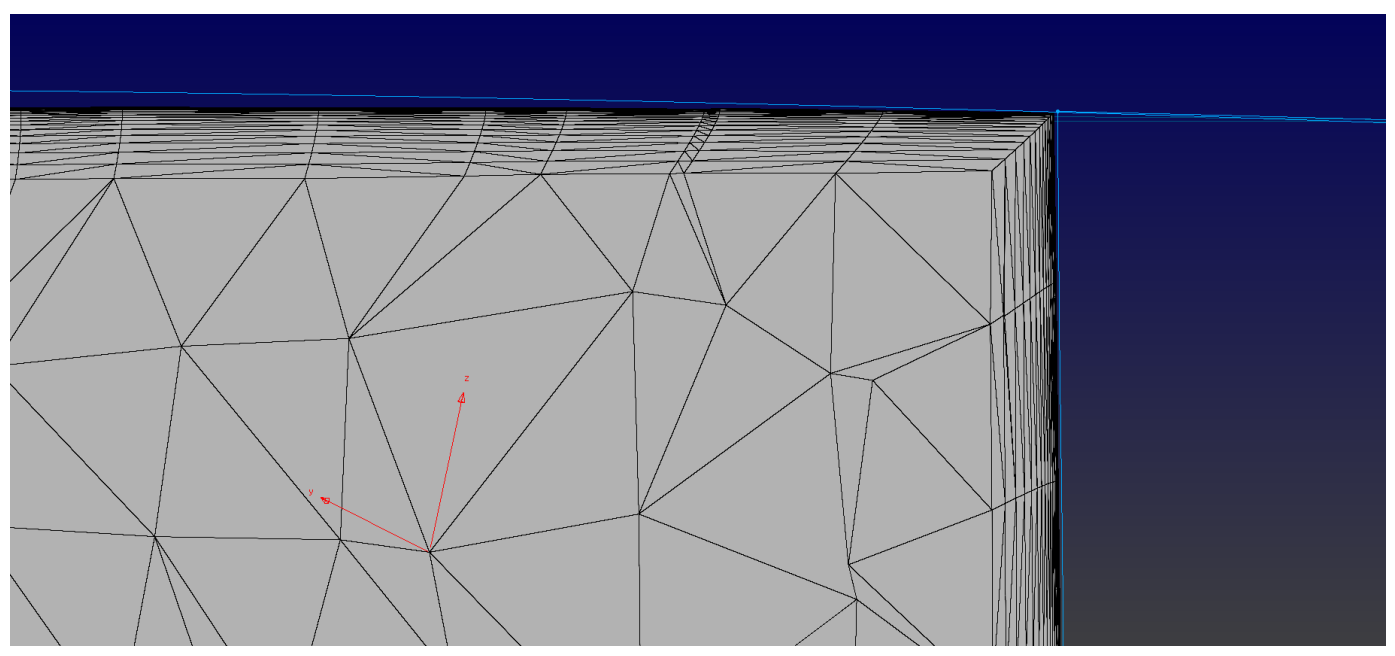

Figure 4.3 Plane cut of volume mesh near a corner. 
After completing the mesh, the boundary and volume conditions are declared. The boundary conditions are set by selecting all the surface meshes that belong to a boundary and assigning them the desired name for that boundary. Similarly, the volume conditions are set by selecting the volume meshes and assigning them a desired name. Two volumes were set for the meshes in this work. The Rotor volume, which was to be set in a rotating frame of reference in the solver, and a Stator volume, to be set in a fixed frame. The Rotor volume had the following boundaries assigned to it: rotor hub, blades, rotor casing, rotor periodic A and B, rotor inflow, and rotor outflow. The Stator volume had the following boundaries assigned to it: stator hub, stator blades, stator casing, stator periodic A and B, stator inflow, and stator outflow. The periodic boundary partners were set as axisymmetric boundary conditions, and the rotor outflow and stator inflow were set as interpolative boundary partners between the Rotor and Stator volumes, also called sliding interfaces. Figure 4.4 is a depiction of the type of boundaries used on the surfaces of the stage mesh, where all the surfaces in color grey are set as solid boundaries. When studs were used in the simulation, another boundary called Studs was assigned to the Stator volume.

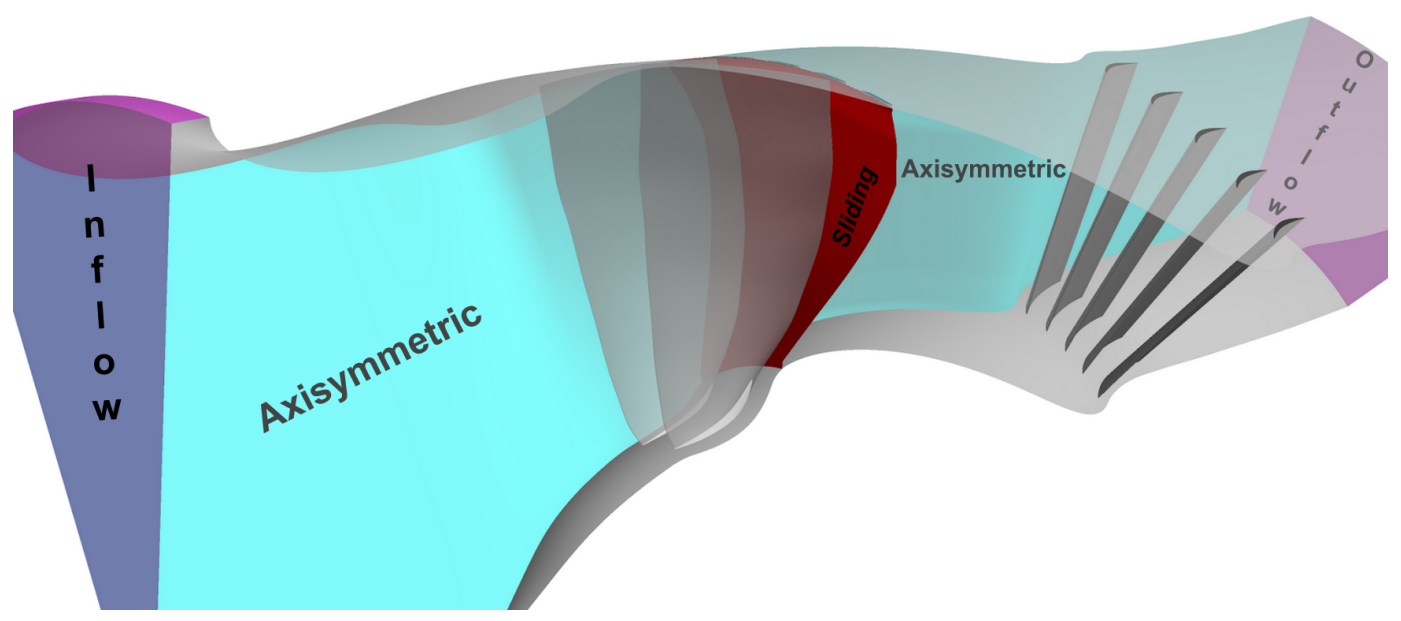

Figure 4.4 Boundary conditions used on surfaces of the stage. 


\subsubsection{Detail of Interpolative Interface and Studs Volume Mesh}

An important feature of the meshes used in this work is the location of the sliding interface and the volume mesh around the studs. In previous work with this turbo-machine performed at the SimCenter, the sliding interface between the Rotor and Stator volumes was located approximately halfway between the fan blades and the stator vanes. Creating surface and volumes meshes near this interface was relatively simple since the components of the stage that possess many details were not in the vicinity. Adding the studs on the casing near the trailing edge of the fan blades for use as casing treatment added a level of complexity for two reasons: (i) the need for a new sliding interface around the location of the studs, and (ii) making use of a separate volume mesh for the regions where studs were to be included.

Adding a second interface for the studs was necessary because the flow solver requires that structures like this be in a stationary frame, and the location of the studs is very near the blades in the Rotor volume, a rotating frame. Creating a separate volume mesh for the near vicinity of the studs had a strategic purpose related to the results of the simulations: any changes in the solutions and the flow-field could be attributed only to the introduction of the casing treatment and not to changes on the rest of the mesh brought about by the introduction of the casing treatment. Having a block that isolated the mesh around the studs allowed introducing the studs, modifying their geometry, and recreating the volume only within that block, while leaving the rest of the stage volume mesh untouched. Figure 4.5 shows (in pink) the sliding interface between the rotor and stator sections, and the sliding interface for the studs alone. 


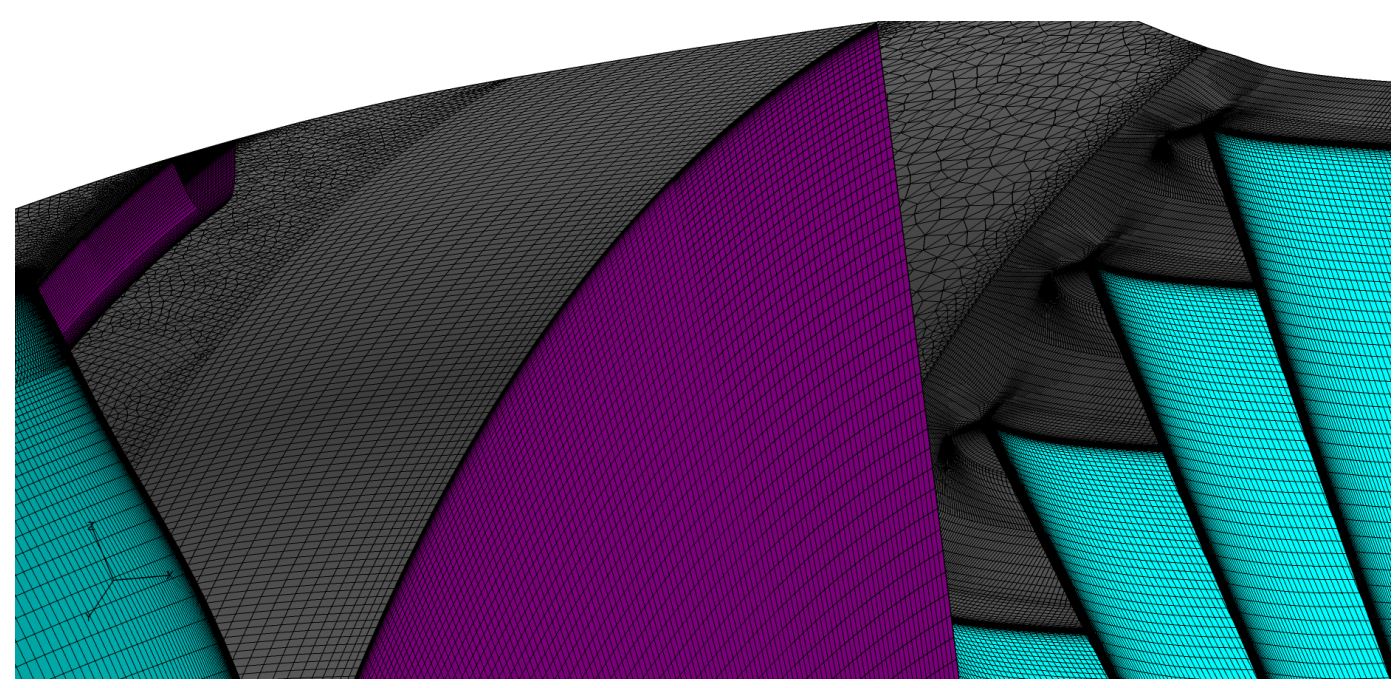

Figure 4.5 The two sliding interfaces used in earlier simulations are shown in pink, rotor blades and stator vanes are shown in light blue, and casing is shown in grey.

Initial simulations with two sliding interfaces were successful but run time increased dramatically. Relative to simulations with a single sliding interface, using two sliding interfaces took approximately $30 \%$ longer to run. This motivated a work around alternative to using two such interfaces. It was decided that the main sliding interface be brought near the trailing edges of the fan blades, such that the studs were now part of the Stator volume instead of being located within the Rotor volume. A separate volume block could still be created for the studs region where the surface meshes defining the block, other than the surface on the casing and the studs, were constant. This allowed merging the studs volume block with the Stator volume exactly the same way every time a modification of the casing treatment was done. Figure 4.6 serves to visualize the details described above. 


\section{Studs Block}
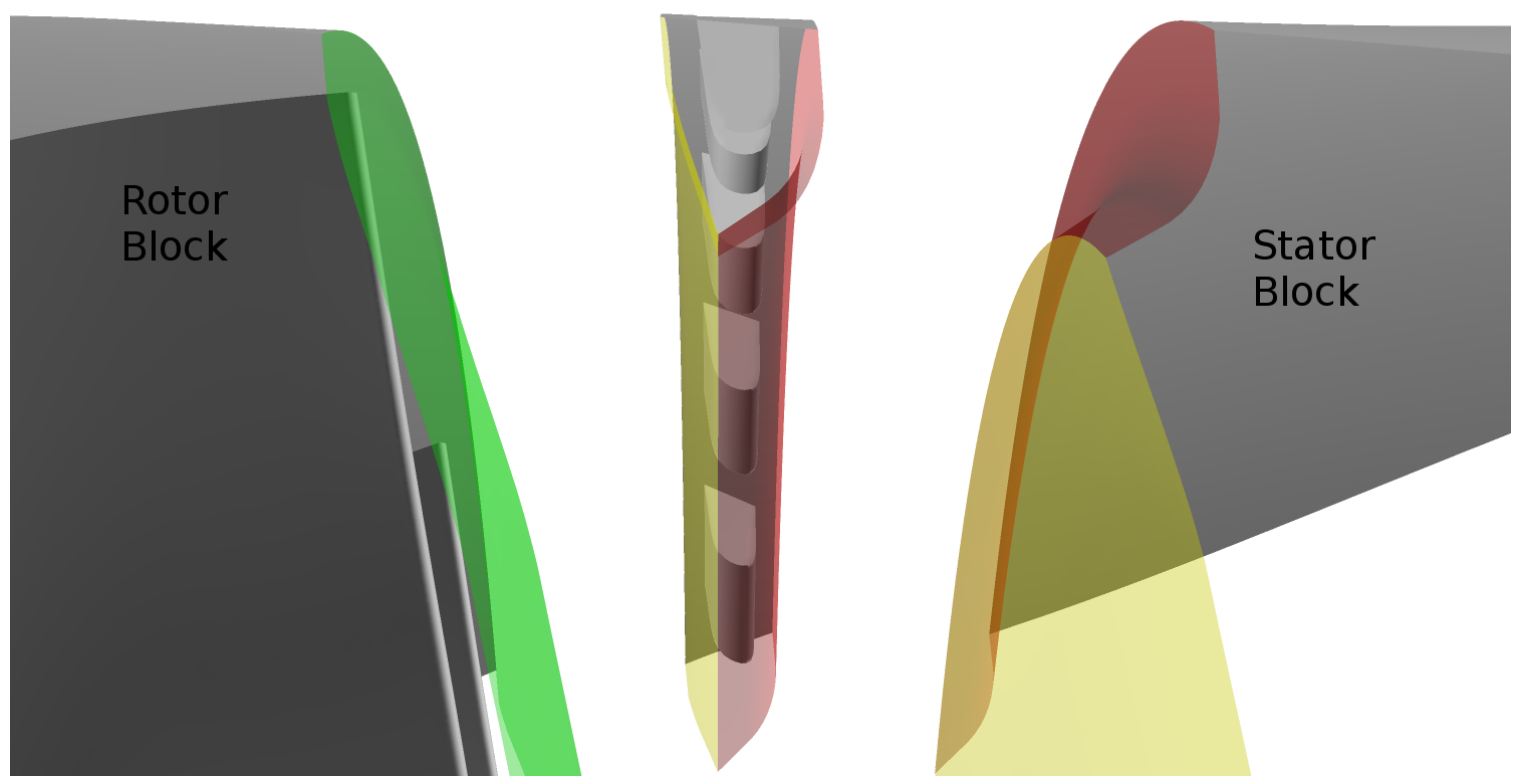

Figure 4.6 Surfaces separating the Rotor, Stator, and Studs volumes.

Figure 4.6 shows the surfaces separating the three blocks described in this section. All grey surfaces are actual solid surfaces. The green, yellow and red (mesh) surfaces remain unchanged with the modifications of the casing treatment geometry. The green surface is the sliding interface on the rotor side. The red surface meshes on each block are identical. When the Studs block and the Stator block are merged, the red surfaces are matched point to point and are unaccounted for once a single block is defined. Finally, once the Studs block and the Stator block are merged into a single one, the yellow surfaces become the single sliding interface on the stator side. When the baseline, smooth casing (no studs), case was tested, the ring shaped volume mesh (the "studs block" in Figure 4.6) contained no studs. 


\subsubsection{Finalizing the Mesh}

Once the generation of the volume meshes is concluded, they can be exported with Pointwise into mesh files. Making the mesh files suitable for the flow solver is a process that requires the use of various software tools developed in-house. First, the meshes are checked for errors (i.e. negative volumes, "orphan" nodes) using the "gridcheck" tool. The next step is to merge the Studs and the Stator volume meshes into the complete Stator volume mesh using the "SUGG" (SimCenter unstructured grid generation) tool. Then, the Rotor and the Stator volume meshes are concatenated using the "gridcat" tool. Once the mesh files are finalized and suitable for simulation, the Rotor volume mesh contains about 7.5 million nodes and the Stator volume mesh contains about 8.6 million nodes, for a total of about 16.1 million nodes for the turbofan stage. Figure 4.7 shows a plane cut of all volume meshes across the sliding interface once they are merged and concatenated.

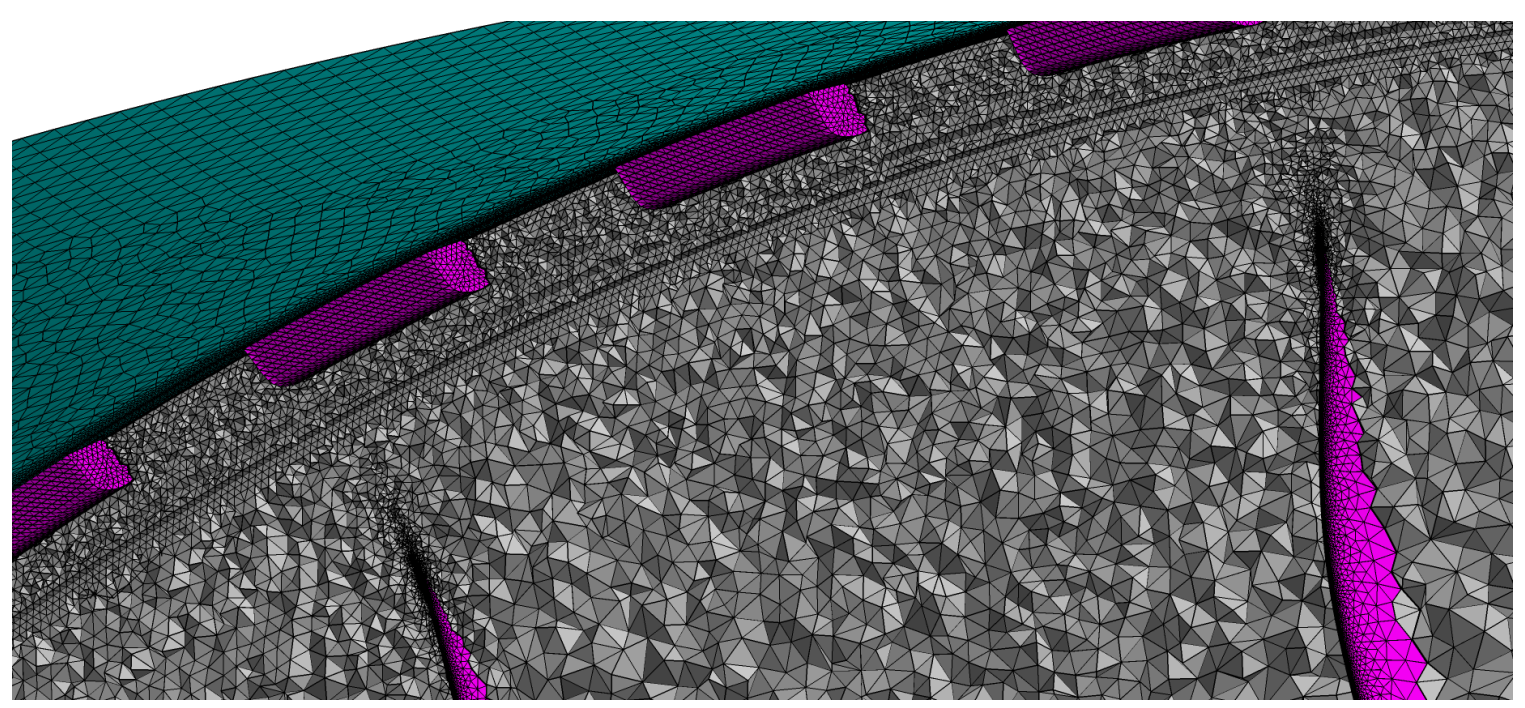

Figure 4.7 Aft-looking-forward view of volume mesh after generation and merging where the casing surface is shown in green, rotor blades and studs are depicted in pink, and a plane cut of the volume mesh is depicted in grey. 


\subsection{Setting Up and Running Cases}

Once a mesh is ready, the next step is creating files that define for the solver the boundary conditions and the parameters the solver should use for performing computations. The SimCenterdeveloped software "TenasiGui" is used for this purpose. TenasiGui is a graphical user interface to the flow solver Tenasi designed to make the creation of the boundary and parameter files easy, and to provide tools to monitor the progress of the solution during run time.

The boundary condition file assigns boundary conditions to the surface boundaries defined in the mesh file. This is where the values of total pressure and total temperature are assigned to the rotor inflow boundary, where the back pressure is defined at the stator outflow, and where the solid walls are defined as adiabatic no-slip surfaces. The periodic surfaces are defined as axisymmetric boundaries, and the rotor outflow and stator inflow boundaries are matched as interpolative partners. This file also defines whether a volume mesh is set in a rotating or stationary frame of reference.

The parameters file defines the parameters the solver will use to perform the numerical simulations. This is where the solver is set to pick up initial conditions from another solution file or if the simulations are to be started from zero velocities in the flow-field, for example. The total number of steps and the time step size are defined here, as well as the number of steps to used first order spatial discretization before switching to second order. Reference values are specified, flow regime, turbulence model, and numerical schemes for solving the governing equations, just to name a few of the input parameters that are commonly needed.

Next is the decomposition of the mesh file, which is done using the SimCenter's "decomp" tool. Required as inputs for mesh decomposition are the mesh file name, the length units used in 
the mesh file, a case name, and the number of subdomains in which to decompose the mesh (which is the number of processors to be used for computation).

Finally, the cases are submitted to an in-house cluster for computation. Once submitted, some general computed values can be monitored from the file with the ".out" extension. Other files that record residuals, changes in solutions, and even timing are available for monitoring. Still other files are available to interact at run time with the solver to, for example, have the solver export solution files at any given time-step, or to terminate the run immediately, if so desired. If the simulation becomes unstable and computations are terminated, the flow solver will export files specifying the coordinate locations in the mesh where variable solutions became undefined or unrepresentable values (not-a-number, $\mathrm{NaN}$ ). Furthermore, when using rotating frames of reference in the simulation, if the solver is unable to find points on a rotating frame, it will suspend the computations and export files providing locations of the "unfound" points.

Once the solver finishes performing computations, information in the files provided by the solver can be extracted and analyzed to help decide whether running the case is concluded. In the present work, this is done by plotting the values of mass flow rate through the duct. When the plot shows the mass flow has remained essentially constant over several revolutions, it is deemed the solutions have arrived at convergence. Otherwise, the solver is re-started using the most recent solutions typically until the mass flow stabilizes. Figure 4.8 is an example of a mass flow plot indicating convergence of solutions. The figure shows that a first simulation of 40 revolutions was ran using 90 steps per revolution. The second simulation ran 4 revolutions using 900 steps per revolution. Finally, a third simulation ran 2 revolutions using 1800 steps per revolution. 


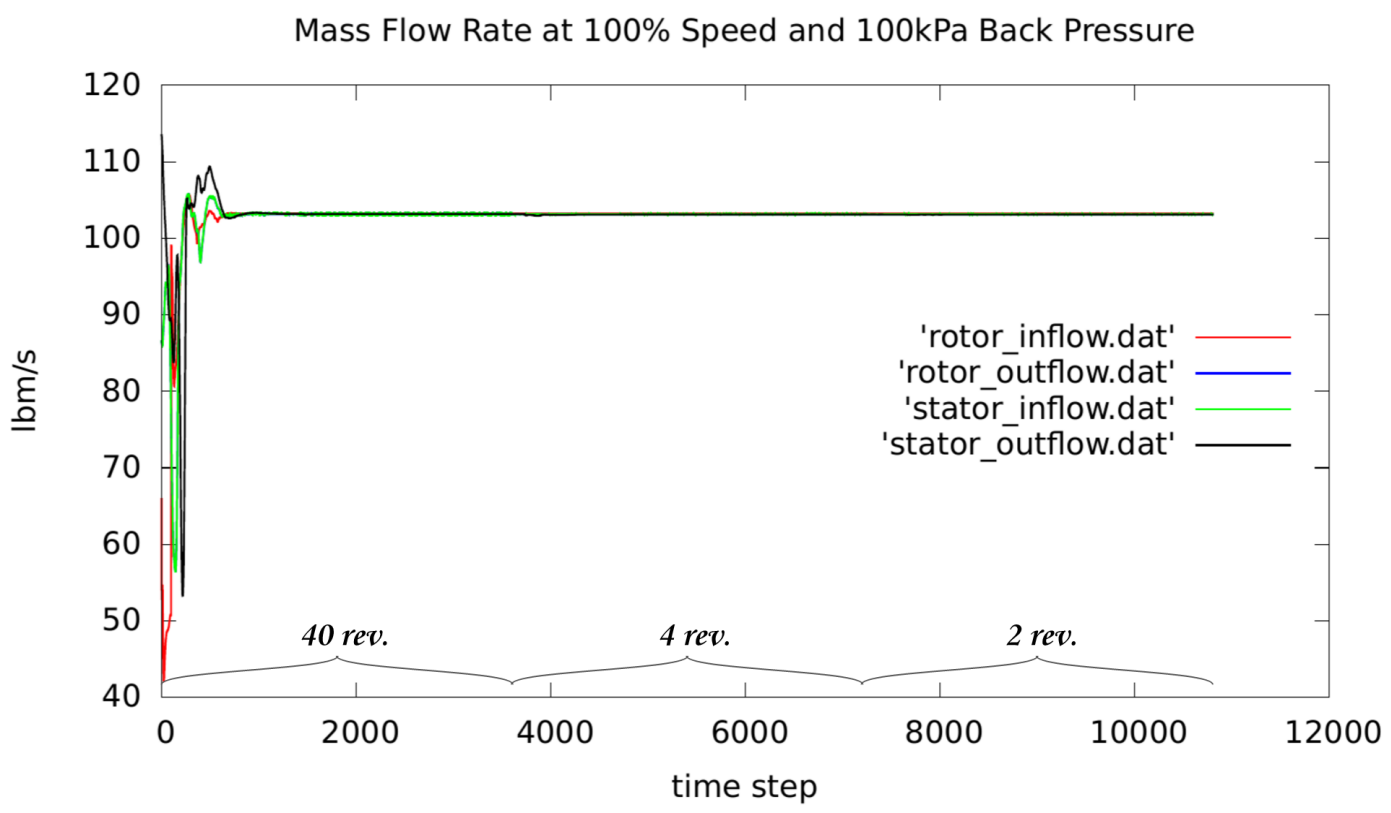

Figure 4.8 Sample of mass flow computed across four boundaries in three consecutive simulations.

\subsection{Post-Processing and Visualization}

Once a case is completed, the solutions are post-processed and analyzed. First, the solution files, provided by each processor used in the computations, are recomposed into a single file using the SimCenter's "recomp" tool. Then, the solutions in the flow-field can be visualized using the FieldView software [36] to observe the details of interest. FieldView can provide visualization of scalar and vector fields, depending on the type of information being analyzed. It also provides tools for extraction of data that may be of particular interest to the user. For example, in this work, such data has been velocity magnitudes, flow directions, pressure and temperature values, among others. GNUPlot [37] was used whenever such kind of data needed plotting for better analysis, and GNUPlot scripts were used to automate the process. Also, extraction of information from solution files was possible through development of Python scripts. 


\section{CHAPTER 5}

\section{RESULTS OF BASELINE SIMULATIONS}

This chapter presents the results of the simulations of the fan stage with a smooth casing. Such results are compared against the experimental measures of performance of the SDT2-R4 to ensure an acceptable degree of agreement. This process is called validation, and achieving it is the first major part of this work and a very important step. The reason is that being able to computationally reproduce the experimental results provides a level of confidence in the results of the simulations for the modified geometry. It can then be said that the findings from this computational study resemble closely what would happen in a real case scenario. At this point, no casing treatment results are yet considered.

The performance values were calculated from simulations on the stage for corrected speeds of $100 \%$ and $87.5 \%$ of the design speed, which is 12,657 RPM. The quantities used to calculate the performance were mass flow, total pressure, and total temperature. Such quantities were integrated at the planes of the stage inlet, at the fan exit rake, and at the stator exit rake, as documented by Hughes [2] and shown in Figure 5.1. Also, the computed values presented here are for a range from the choke point (maximum mass flow) to a mass flow rate similar to that presented in the experimental results $(92.1 \mathrm{lbm} / \mathrm{s}$ approximately). 


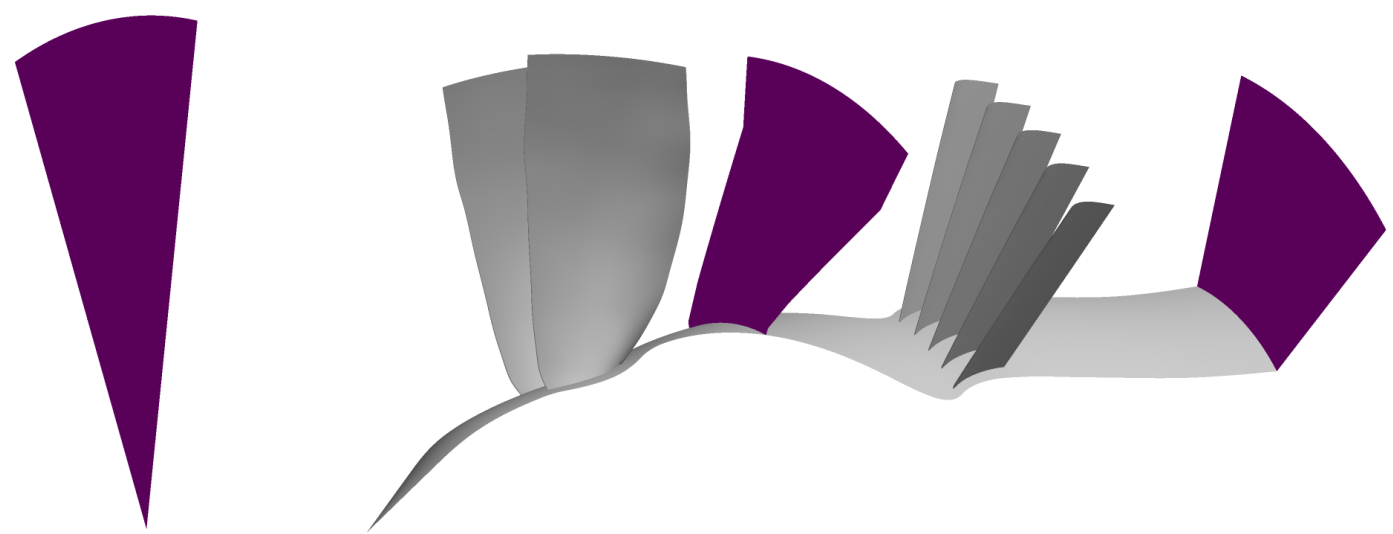

Figure 5.1 Planes of integration of mass flow, total pressure and total temperature.

\subsection{Performance Maps}

\subsubsection{Fan Performance}

The pressure ratio, temperature ratio, and adiabatic efficiency curves for the fan rotor only are given in Figures 5.2, 5.3 and 5.4, respectively. The values used for their calculation were integrated at the stage inlet and at the fan exit only. The values of adiabatic efficiency were calculated using total pressure and total temperature values, and equation 5.1. The experimental values were obtained from Hughes [2].

$$
\eta_{a d}=\frac{\left(p_{2} / p_{1}\right)^{(\gamma-1) / \gamma}-1}{\left(T_{2} / T_{1}\right)-1}
$$

The performance curves from the simulation are observed to closely resemble the experimental results with a slight over-prediction at both speeds. The trends of the pressure ratio curves are similar, only shifted up by an average of $0.95 \%$ for the $87.5 \%$ speed line and $0.50 \%$ for the 
$100 \%$ speed line. In the case of temperature ratios, the over-prediction in the $87.5 \%$ speed line is of about $0.28 \%$, and $0.18 \%$ for the $100 \%$ speed line.

One of the main reasons for these discrepancies can be attributed to the computational mesh. This is concluded from considering previous studies performed by other authors using the same flow solver with the same parameters. In the works by Hyams [27] and Lin [3], simulations were carried out on the same turbofan model and using Tenasi. Each author used a different mesh that was tailored to the purposes of their study. Their results, although resembling closely the data from the experiments, had some over or under prediction as is the case here. In this work, a mesh study showing solution convergence by mesh refinement is not in order since there were other challenges to overcome (i.e. working sliding interface, matching experimental data). However, two volume meshes created using the same surface mesh but different number of viscous layers were tested obtaining different results. More insight on this is provided in section 5.3, but it can be said here that a better approximation of the experimental results can be obtained by varying the number of viscous layers and, also, by choosing a viscous spacing based on different fan speeds.

The computed curve for adiabatic efficiency of the fan matches very well the efficiency curve obtained in the experiments, as shown in Figure 5.4. In spite of the over-predictions observed in the computed results of pressure and temperature ratios, the agreement in efficiencies does not come as a surprise since such over-predictions would be expected to partially balance through the fraction in equation 5.1. Overall, the results obtained for the fan performance can be considered good, since the curve trends are very similar and the discrepancies are very small. 


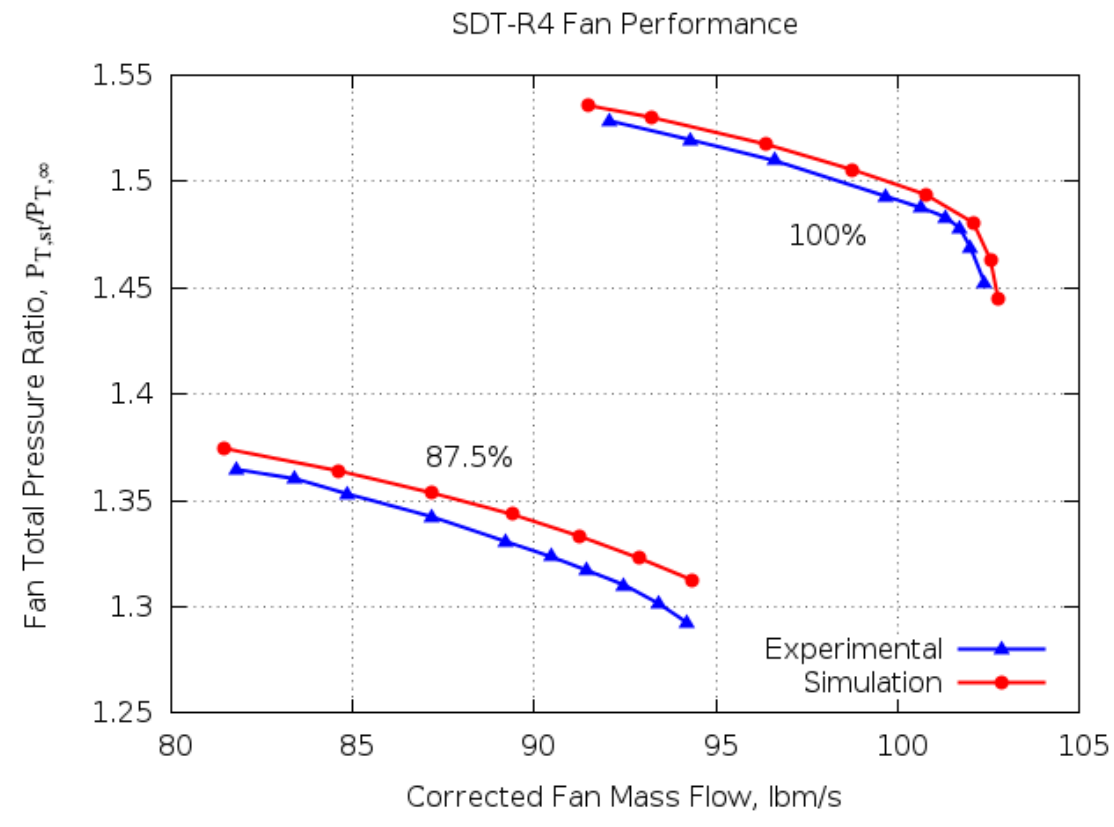

Figure 5.2 Fan total pressure ratio.

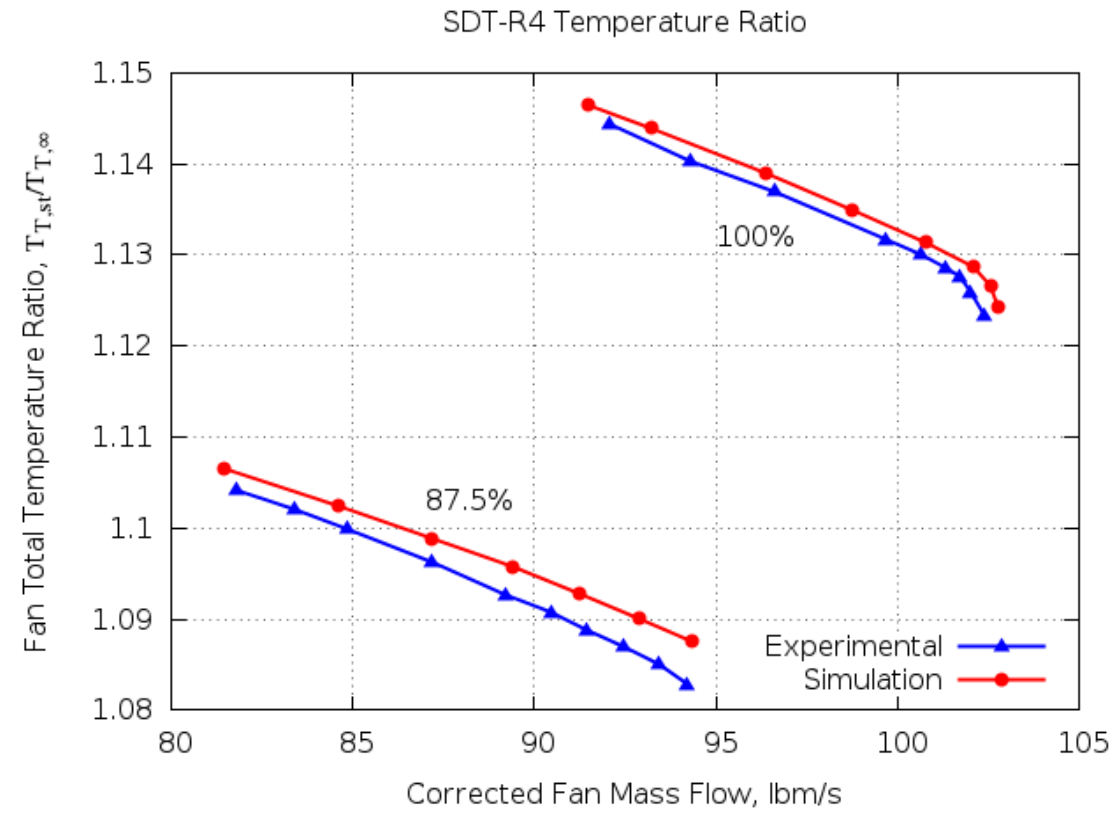

Figure 5.3 Fan total temperature ratio. 


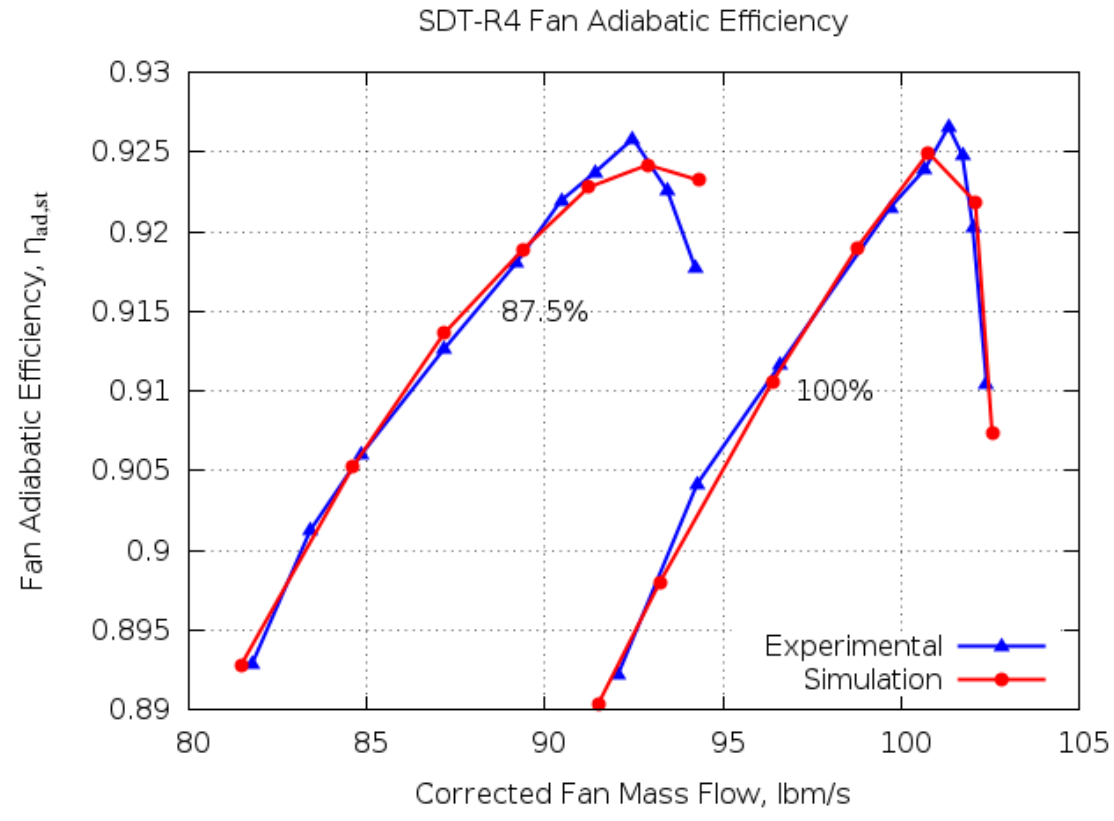

Figure 5.4 Fan adiabatic efficiency.

\subsubsection{Stage Performance}

The total pressure ratio curve for the stage is given in Figure 5.5. In the case of the $100 \%$ speed line, the agreement is very good. This is not quite the case for the $87.5 \%$ speed line where the pressure ratio at the choke point is over-predicted by about $1.46 \%$, but the difference diminishes as the mass flow rate decreases.

The red curves in Figure 5.6 show the computed results of adiabatic efficiency. It is observed that the shape of the $100 \%$ computed curve is very similar to the experimental one and they overlap with a somewhat differing result for the last point closer to stall. This is not the case for the $87.5 \%$ speed line, where only the trend of the curve is similar. Most of the values here have some level of disagreement, with the largest one being of $0.97 \%$ at about $91.3 \mathrm{lbm} / \mathrm{s}$ mass flow. 


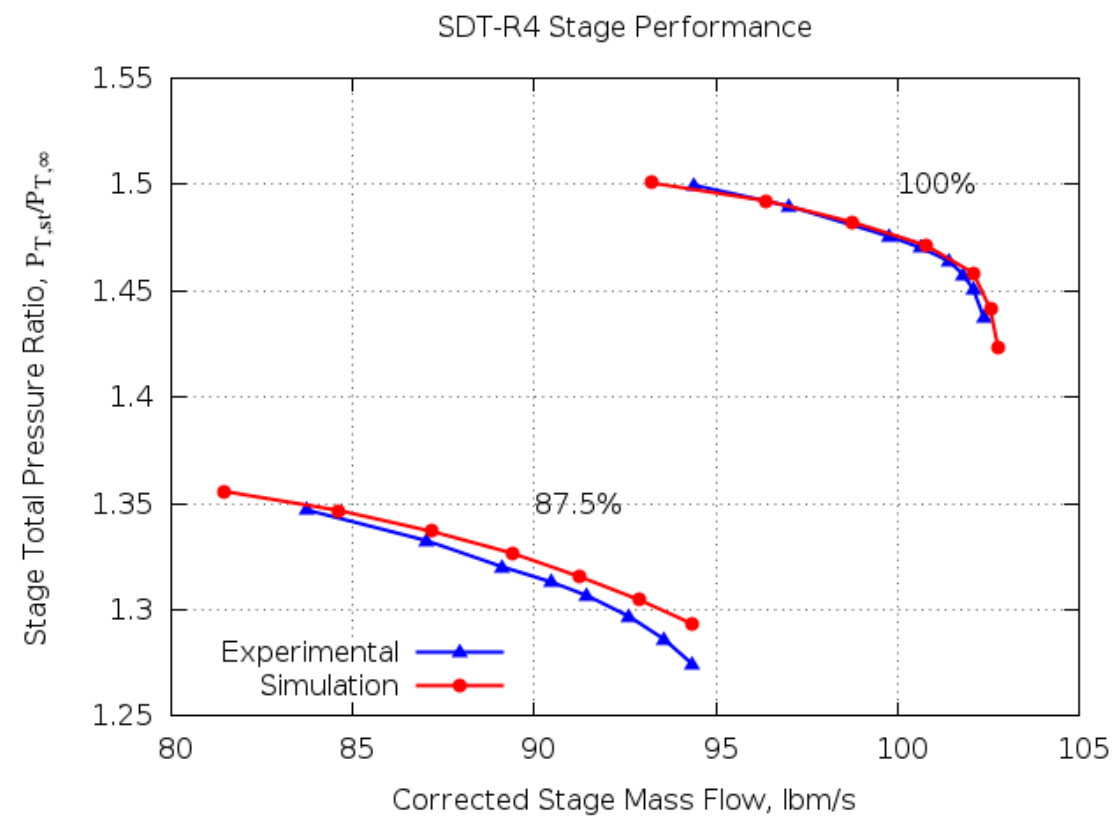

Figure 5.5 Stage total pressure ratio.

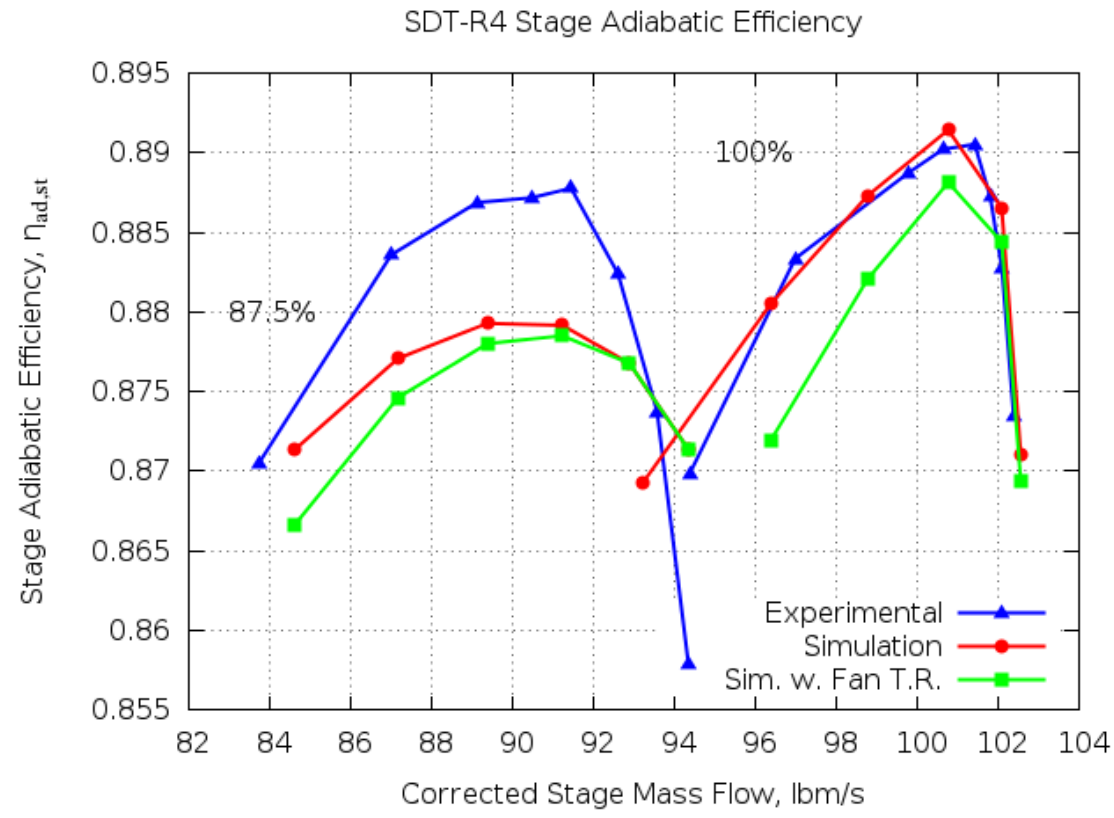

Figure 5.6 Stage adiabatic efficiency. 
One important aspect is to be noted about the calculation of adiabatic efficiency. According to the report of the experimental results [2], the calculation of the adiabatic efficiency for the stage was performed using the temperature ratios for the fan since the temperature ratios for the stage were not obtained (therefore not presented). In the experiments, the temperature ratios were not expected to change significantly across the stator vanes. However, the adiabatic efficiency curves for the stage presented here in red were calculated using a temperature ratio calculated across the entire stage. The stage adiabatic efficiency curves calculated using the fan temperature ratio are provided in green and do not agree very well with the reported results. It is observed that at the choke point the disagreements are not big, but become greater as the mass flow rate decreases and the curve moves toward the near stall region (left side). This observation actually correlates with the plot of temperature ratio changes across the stage for the $100 \%$ speed case, presented through Figure 5.7. In this figure, it is seen that there is indeed a decrease in temperature ratio as the flow passes through the stator, which is greater for flow conditions near stall. A similar point is made by Hyams [27]. For this reason, and simply by the fact that temperature ratios across the stage are available for the adiabatic efficiency calculation, it is decided that adiabatic efficiencies are calculated this way for the rest of this work.

Finally, three notes are made on the mass flow rates. First, solutions given in this chapter are on mass flow ranges similar to the ones reported for the experiments. Second, the difference between computed mass flow across inlet and outlet surfaces, also known as mass imbalance, is very small for all tested cases, as shown in Figure 5.8. This adds confidence to the solver and the computational mesh validation. Third, it cannot be said that stalled flow conditions were captured in the simulations in this work because only a portion of the turbofan wheel was simulated, and 
because stall point conditions were not reported (or captured) in the experiments. However, the entire simulated flow range shall be reported when presenting a comparison of results with casing treatment, and included will be the last experimental point of stable flow-field which will be used as a reference for range extension.

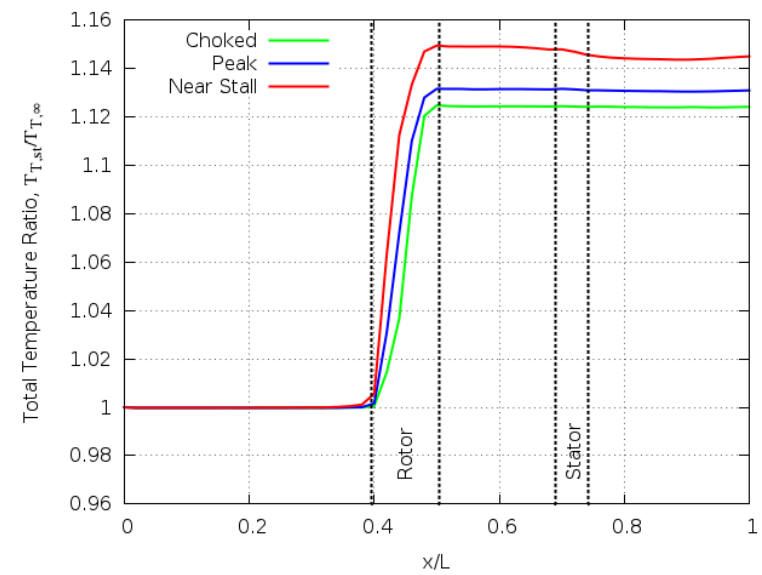

Figure 5.7 Temperature ratio across stage for choked flow conditions, peak efficiency, and near stall at $100 \%$ speed.

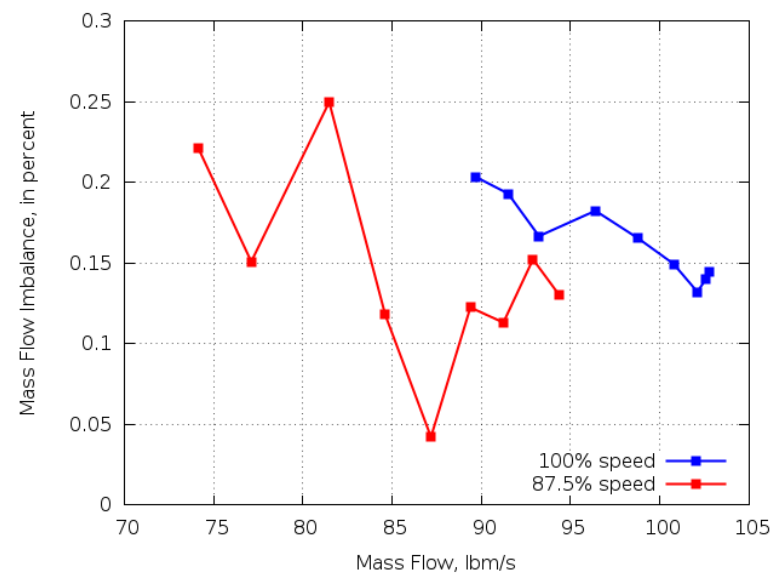

Figure 5.8 Mass imbalances at solution termination where the values are presented as a percentage of mass flow through passage. 


\subsection{Analysis of Flow Field}

In the present section the simulated flowfields at design speed are visualized through some of the variables solved in the governing equations and other quantities calculated from these solutions. The goal of this exercise is two fold. The first goal is to correlate the solutions to the physical behavior of the flow-field at various conditions; the second goal is to observe details of the flow-field and gain insight on flow phenomena.

\subsubsection{Location of Shocks by Operating Condition}

Figure 5.9 displays the surface of the turbofan section colored by static pressure at different flow conditions. The flow conditions were varied by setting the static pressure at the outlet surface (back pressure) to values that emulate the flow conditions at the choke point, at peak efficiency, and at a near stall condition. The choked operating condition corresponds to the right-most point in the 100\% speed red curve in Figure 5.5. The computed peak efficiency condition corresponds to the highest point in the $100 \%$ speed red curve in Figure 5.6. The computed near stall flow condition would correspond to the last point on the left side of the $100 \%$ speed performance curve. This operating point is usually the last that displays a stable flow rate before setting the back pressure to a slightly higher value that causes a dramatic drop in in mass flow rate. The computed performance curves shown in Figures 5.5 and 5.6 are limited only to the range of the curves provided in the report of the experiments [2] in order to better compare the solutions. The entire computed curves will be included when reporting the performance curves using the casing treatment.

From the pressure coloring across all surfaces, the changing of the flow conditions is observed as it passes through the blade structures. Starting with the stators, it is seen that there is 
an increase in pressure across that row, which is gradual but greater as the flow conditions move from choked to near stall. This is expected since the stator blades are designed to work as diffusers, slowing down the flow to provide a contribution in the static pressure rise.

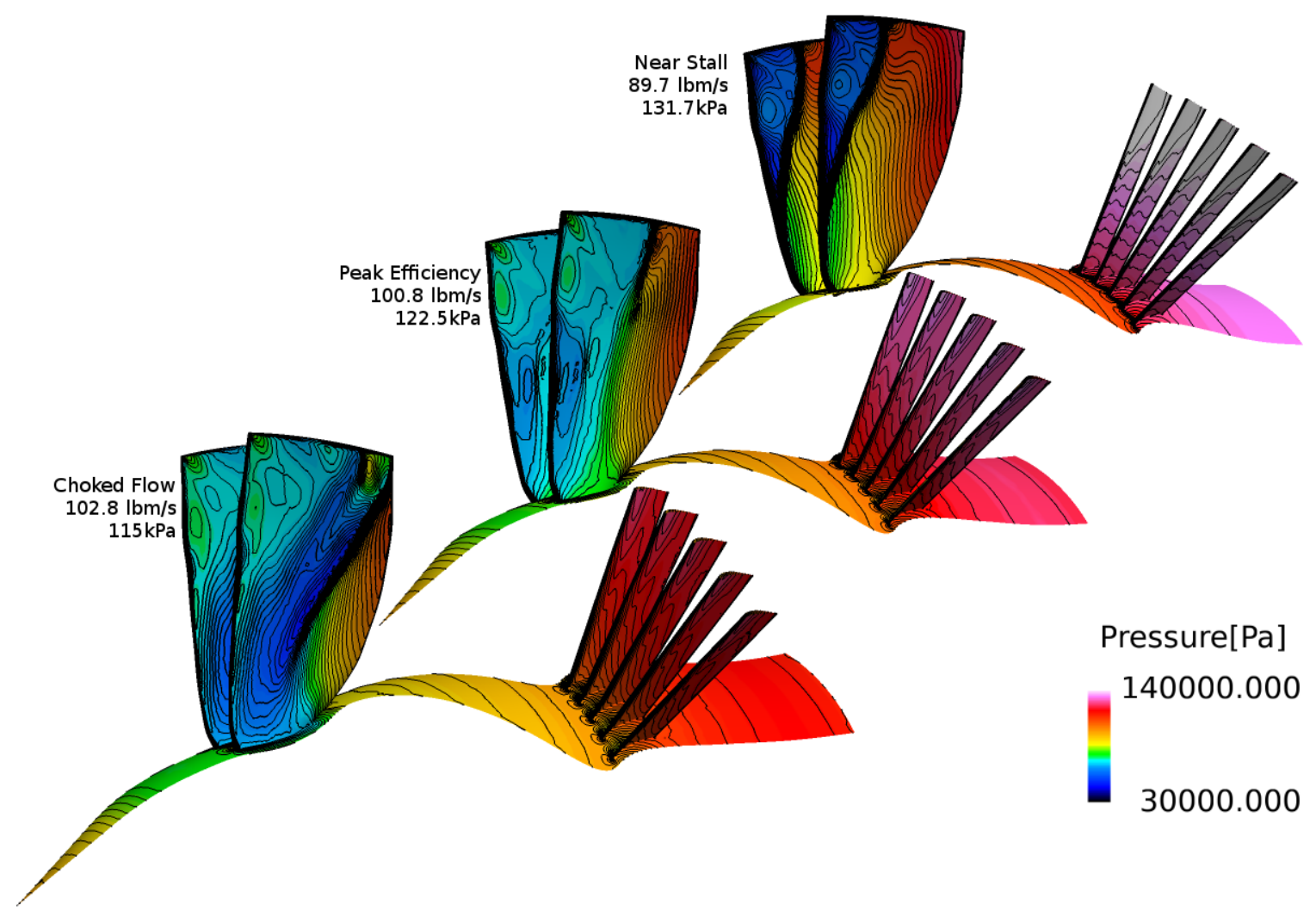

Figure 5.9 Stage surfaces colored by static pressure at different flow conditions.

As this is a transonic fan, various shocks can be spotted on the fan blade surfaces which diverge in extent and location based on flow conditions. In the choked flow condition, one large shock is located across a significant portion of the blade, and a small one is near the tip. The large shock starts at a location about $30 \%$ of the span and $50 \%$ of the chord, and extends diagonally to the tip at the trailing edge. The small shock appears at about $85 \%$ span and at about $20 \%$ chord forward 
of the trailing edge. Visualizations of radial cuts of density at $60 \%$ and $95 \%$ span are provided in Figures 5.10a and 5.12a, respectively, to correlate the above mentioned descriptions with the flow through the passage and upstream of the stage. At $60 \%$ span, a well defined shock is located in the passage at about $25 \%$ chord from the trailing edge on the suction side, which corresponds to the large shock mentioned above. A region of high density change near the leading edge of the blade is also spotted which gives an indication of the formation of a shock as approaching the tip of the blade. In the $95 \%$ span view, the large shock across the passage is observed to be very much at the exit of the passage, and an oblique shock from the leading edge of the blade makes its way across the passage to interact with the adjacent blade suction surface at about $20 \%$ chord distance from the trailing edge.

Back to the surface for peak efficiency in Figure 5.9, it is observed that a shock located about $35 \%$ chord distance from trailing edge, clearly extends from about $65 \%$ span up to the tip. This correlates to Figures $5.10 \mathrm{~b}$ and $5.12 \mathrm{~b}$. At $60 \%$ span, a distinguishable shock is not observed, although certainly regions of rapidly changing density across the passage and at the blade leading edge are present. At $95 \%$ span, it is observed that a shock forms at the leading edge of the rotor fan and extends across the passage to impinge as an almost normal shock on the adjacent blade suction surface.

In the near-stall surface in Figure 5.9, a strong shock is observed extending diagonally from near the root of the fan blade at the leading edge to approximately $45 \%$ chord distance from the leading edge at the tip. At $60 \%$ span in Figure 5.10c, a shock about 25\% from the leading edge is spotted interacting with the suction surface of the blade. At the $95 \%$ span in Figure 5.12c the shock is seen to be fully formed interacting with the suction surface an approximate angle of $100^{\circ}$ and 
slightly upstream of the leading edge of the adjacent blade. Overall, it is observed that the shock moves forward as the back pressure is increased (driving the flow to near stall conditions).

Figure 5.12 provides a similar visualization to Figures 5.10 and 5.11, except the radial cut is at $98 \%$ of the span. The shock structures have changed from the ones observed in Figure 5.11, but not by much. For the choked flow condition, the shock starting at the leading edge has become more oblique at this radial location, and it interacts with the adjacent blade suction side a little closer to the trailing edge compared to the $95 \%$ span location. Also, the shock in the passage has moved further downstream to where it seemed as if it did not interact much with the suction blade side. In the computed peak efficiency flow condition, the shock just upstream the leading edge of the blade has turned slightly oblique across the passage but still interacts in a direction normal to the adjacent blade suction surface. In the near stall flow condition, the angle at which the shock interacts with the suction blade surface has increased to about $102^{\circ}$. 


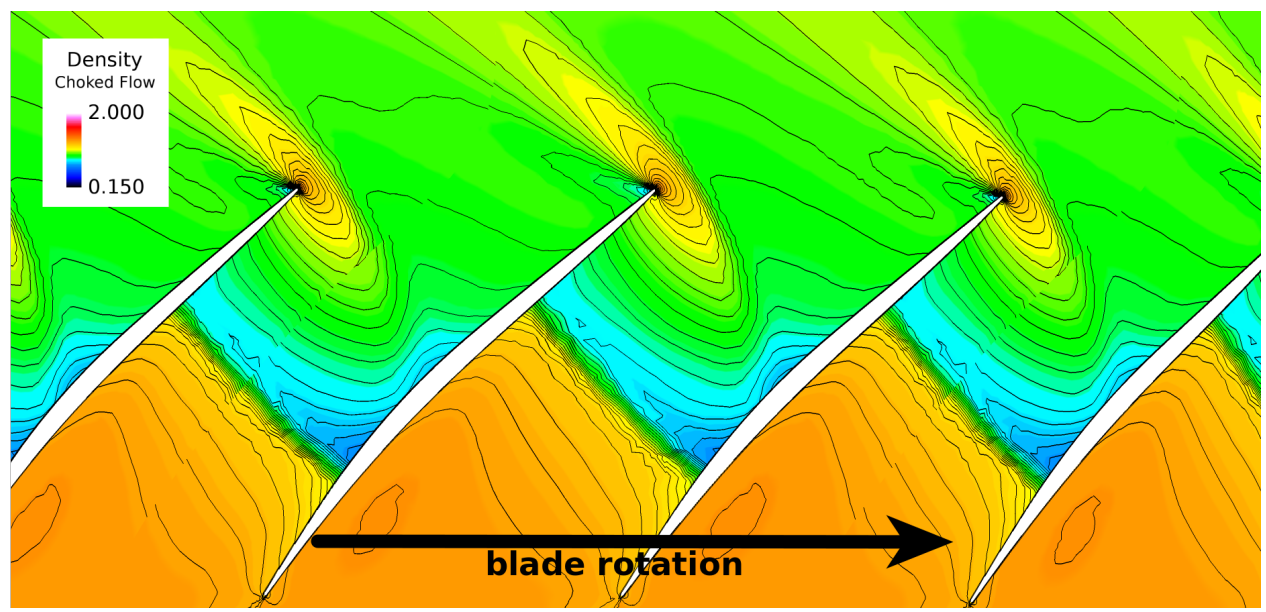

(a) Choked Flow

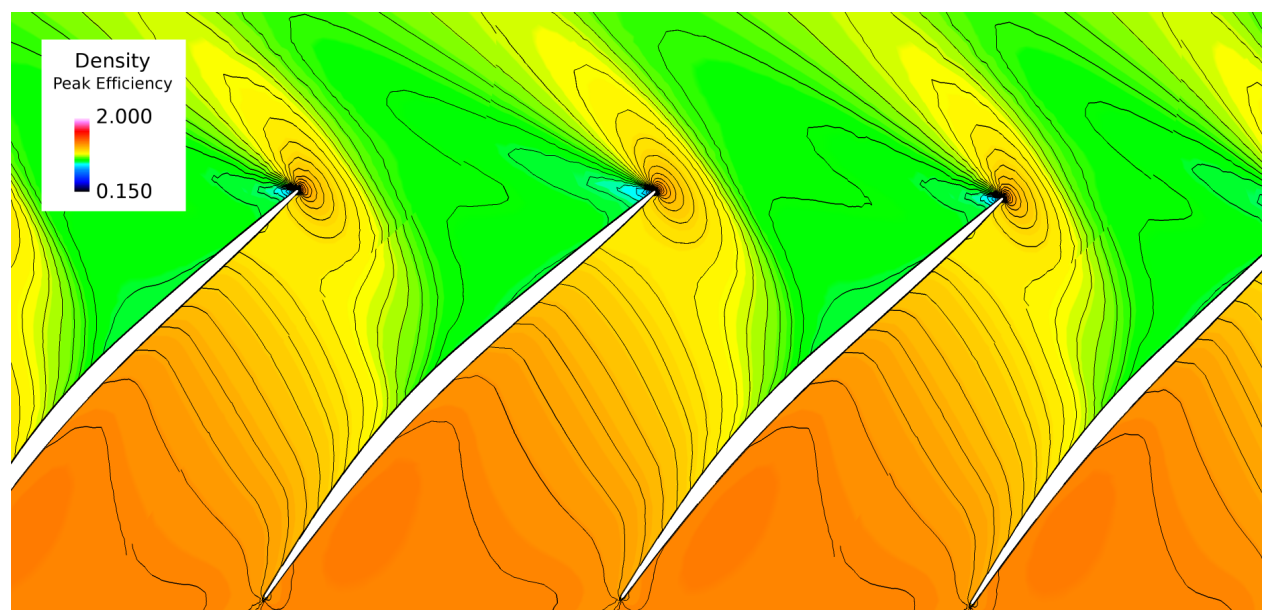

(b) Peak Efficiency

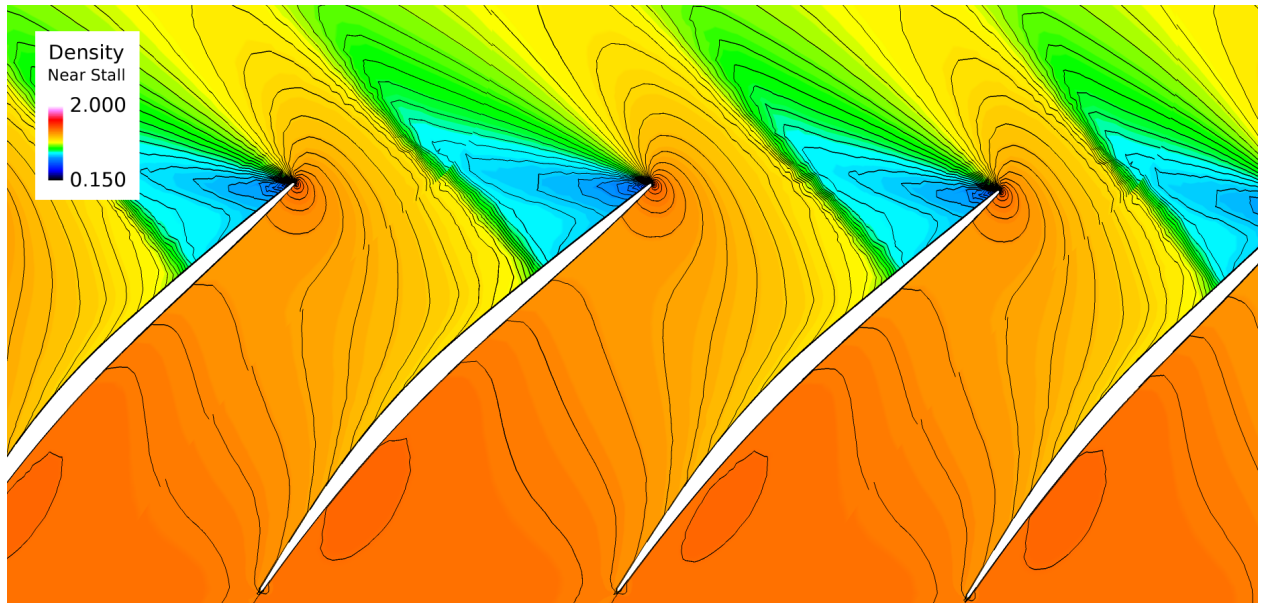

(c) Near Stall

Figure 5.10 Radial cut at $60 \%$ span colored by density (in $\mathrm{kg} / \mathrm{m}^{3}$ ) for three back pressure conditions. 


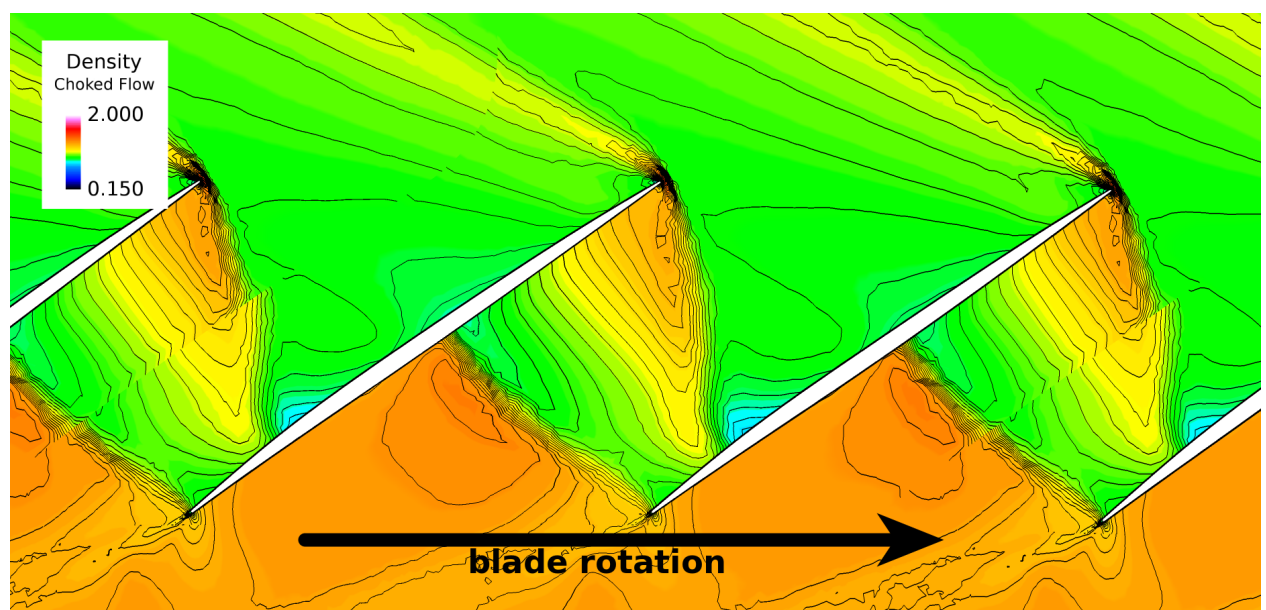

(a) Choked Flow

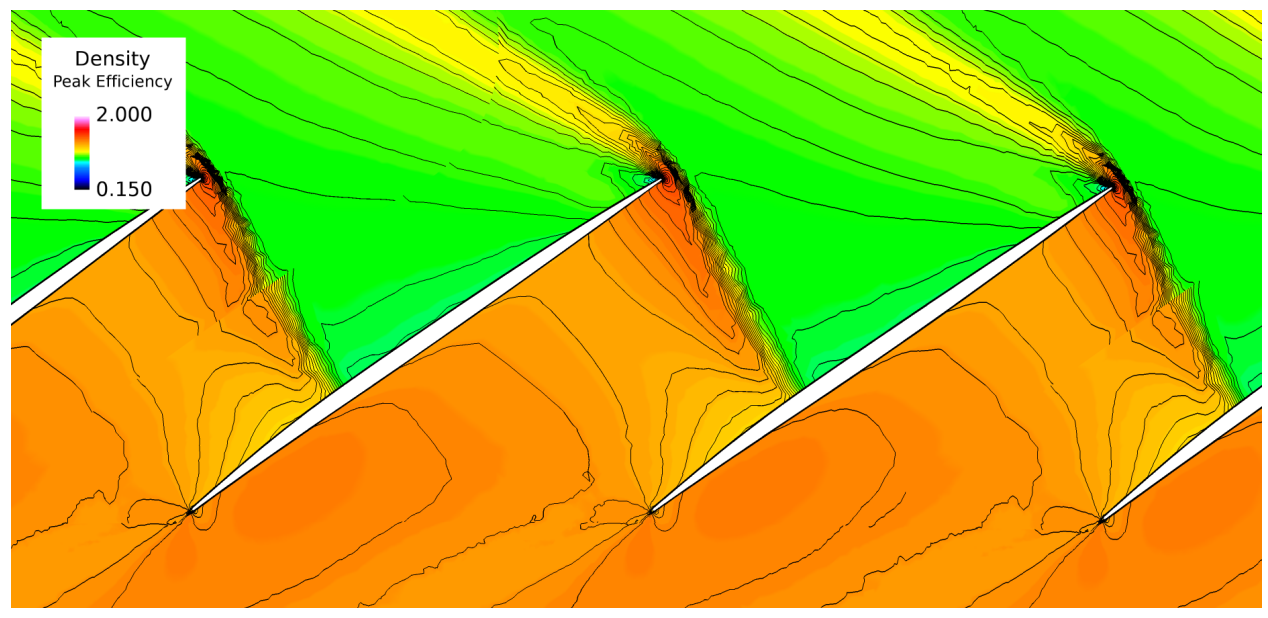

(b) Peak Efficiency

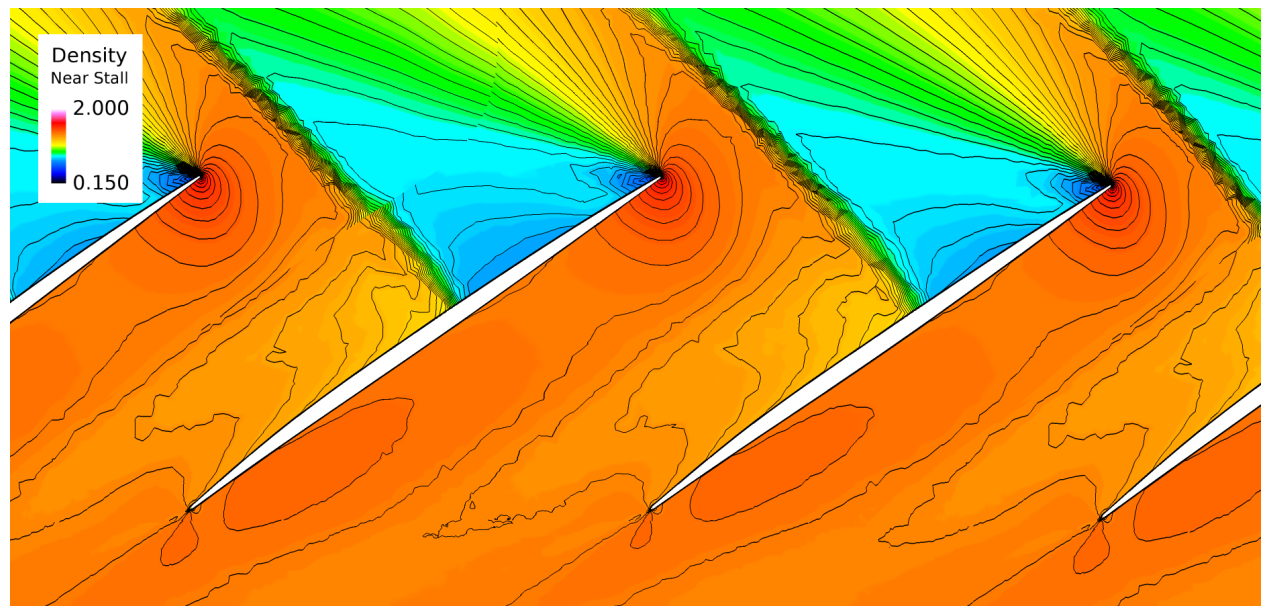

(c) Near Stall

Figure 5.11 Radial cut at 95\% span colored by density (in $\mathrm{kg} / \mathrm{m}^{3}$ ) for three back pressure conditions. 


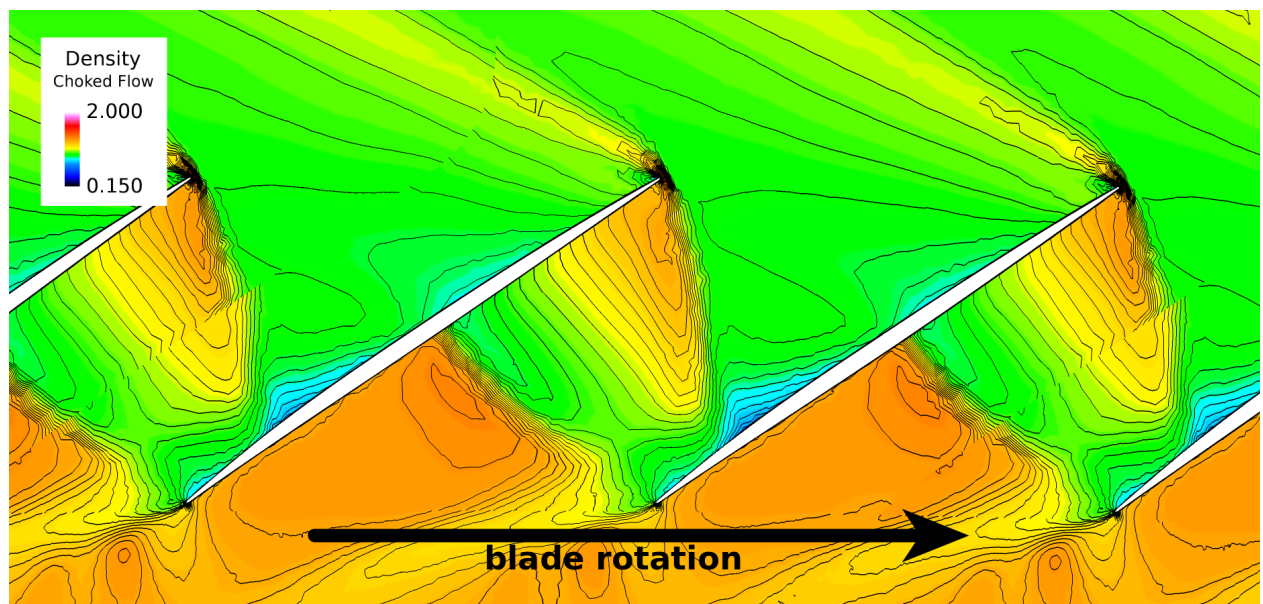

(a) Choked Flow

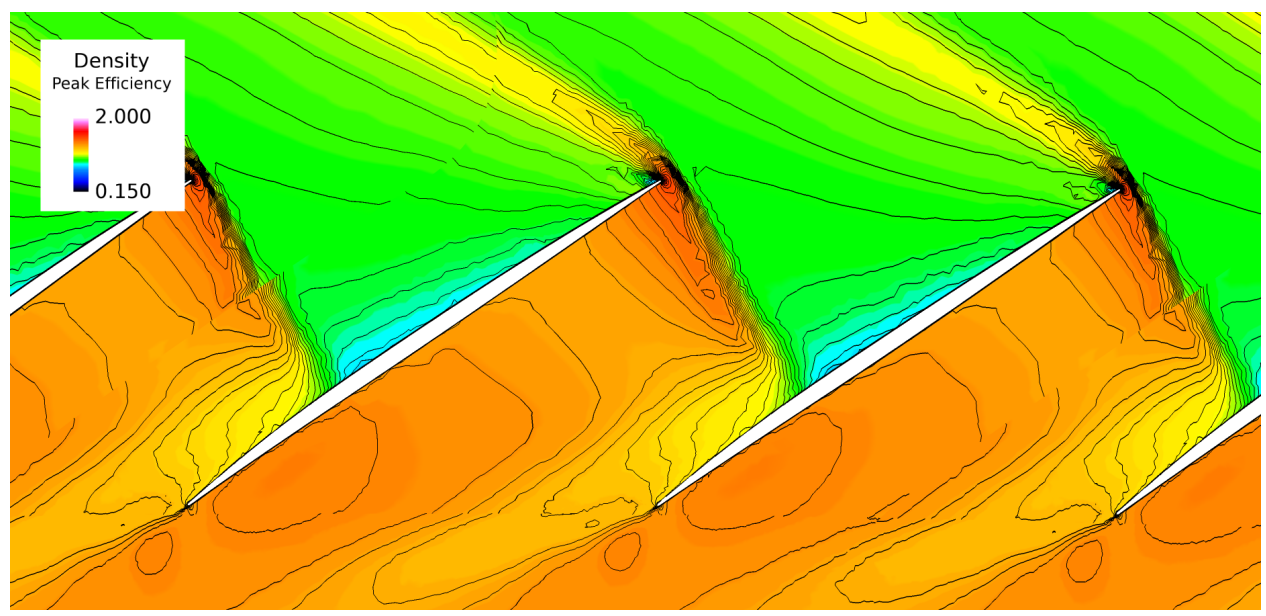

(b) Peak Efficiency

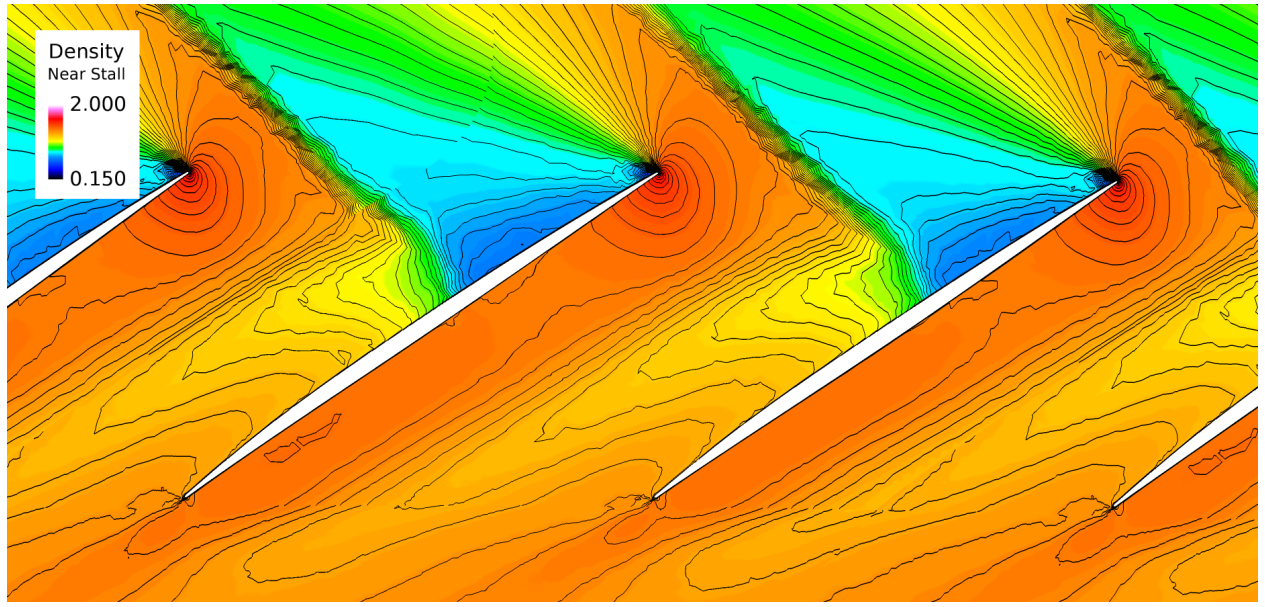

(c) Near Stall

Figure 5.12 Radial cut at $98 \%$ span colored by density (in $\mathrm{kg} / \mathrm{m}^{3}$ ) for three back pressure conditions. 


\subsubsection{Low Momentum Regions by Operating Condition}

Figure 5.13 is given to introduce the issue which creates the interest for working on stability enhancing methods. A location that causes flow instability is observed, occurring predominantly downstream of the passage shock, initiated by the interaction between the tip gap flow (a secondary flow) and the boundary layer buildup on the casing. Figure 5.13 shows radial cuts colored by relative Mach number at slightly different locations for each flow condition, all very close to the casing. The cut for the choked flow (left) is at $97.98 \%$ span, the cut for the computed peak efficiency flow (center) is at $98.50 \%$ span, and the cut for the near stall flow condition is at about $99.95 \%$ span. These radial locations were chosen in order to place the cuts where a spot with the minimum value was observed in the region of low relative Mach number. The red stream lines were constructed using relative velocities and were seeded in the spots of minimum relative Mach number.

The tip gap flow is fluid moving through the space between a blade tip and the casing, and this flow is driven by the pressure difference between the pressure side and the suction side of a blade. When adverse pressure gradients in the axial direction increase (conditions move toward stall, mass flow decreases), a boundary layer buildup forms just downstream of the passage shock, which is a region of low momentum flow than can be visualized through the blue regions in Figure 5.13. In this figure, the operating conditions computed are choked flow on the left $(115 \mathrm{kPa}$ back pressure), computed peak efficiency at the center (122.5kPa back pressure) and computed near stall on the right (131.7kPa back pressure). When a portion of the tip gap flow interacts with this region, it turns into a vortex that expands, and, at high back pressures, forms an unstable flow region also known as stall cell. 


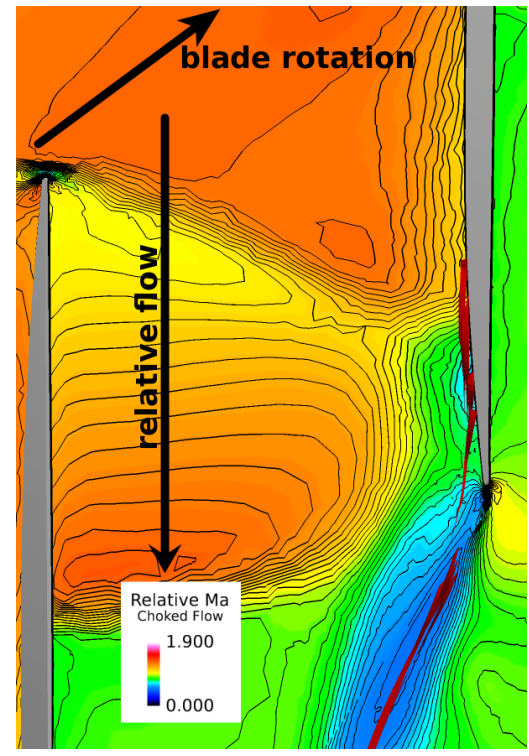

(a) Choked Flow

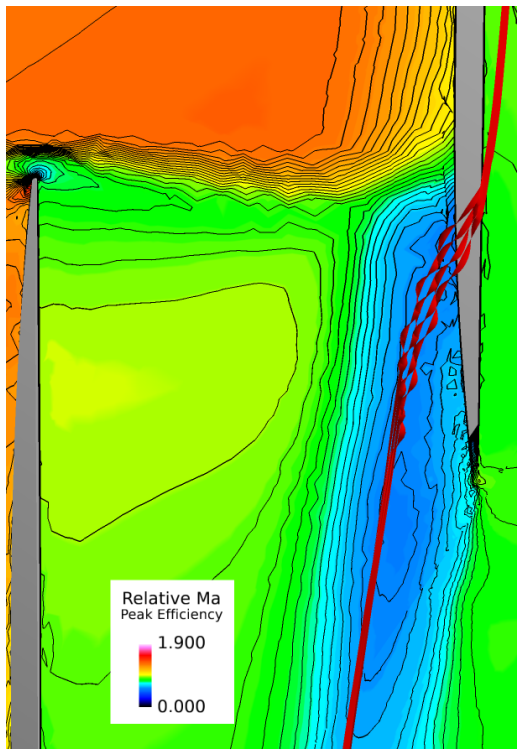

(b) Peak Efficiency

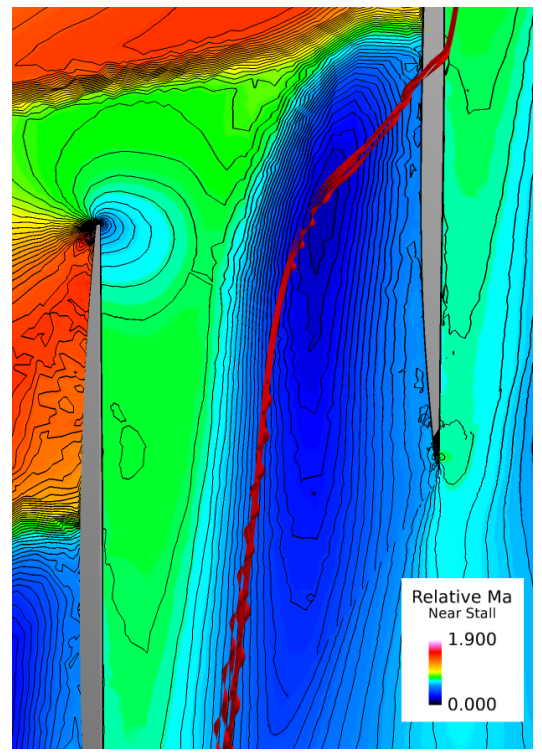

(c) Near Stall

Figure 5.13 Stream lines and radial cuts of solution field colored by relative Mach number.

In Figure 5.14, the same radial cuts from Figure 5.13 are used to display relative velocity vectors. Relative velocity is the velocity of the flow as observed from the point of view of the fan blades. In the right-most picture, corresponding to the near-stall flow condition, a region where the flow decelerates and has a tangential direction component is observed near the suction side of the blade at the right side of the picture. Finally, Figure 5.15 shows multiple planes of constant axial locations colored by relative Mach number. The point of view for the three flow conditions (choke at left, peak efficiency at center and near stall at right) is the same, but the location of the planes are different. Also, the stream lines shown are the same as in Figure 5.13. The purpose of Figure 5.15 is to have a three dimensional view of the low momentum regions under consideration. 


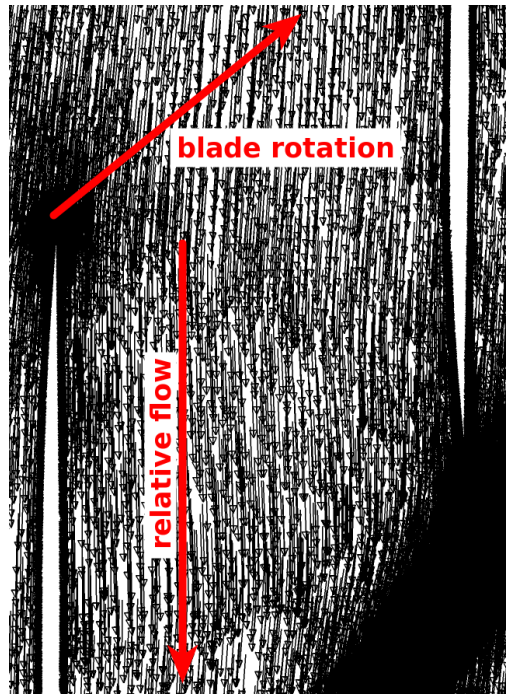

(a) Choked Flow

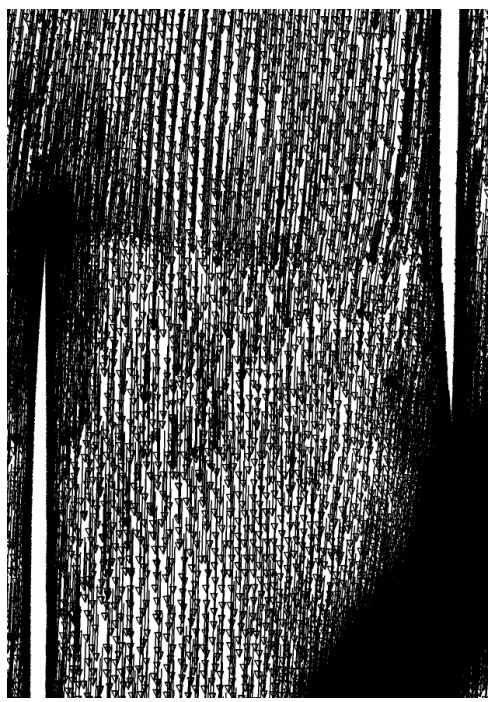

(b) Peak Efficiency

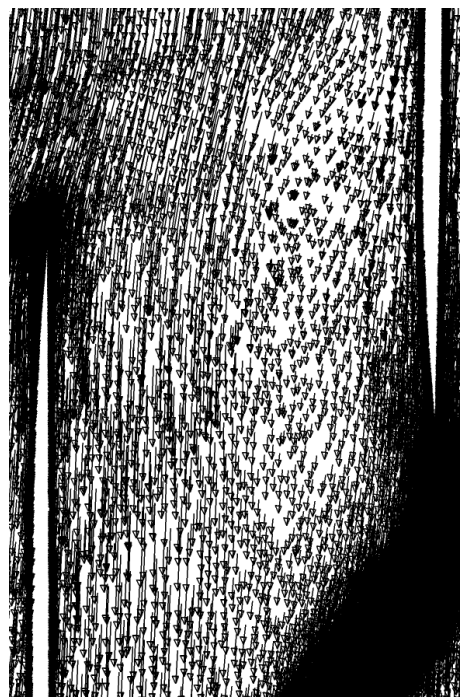

(c) Near Stall

Figure 5.14 Relative velocity vectors.

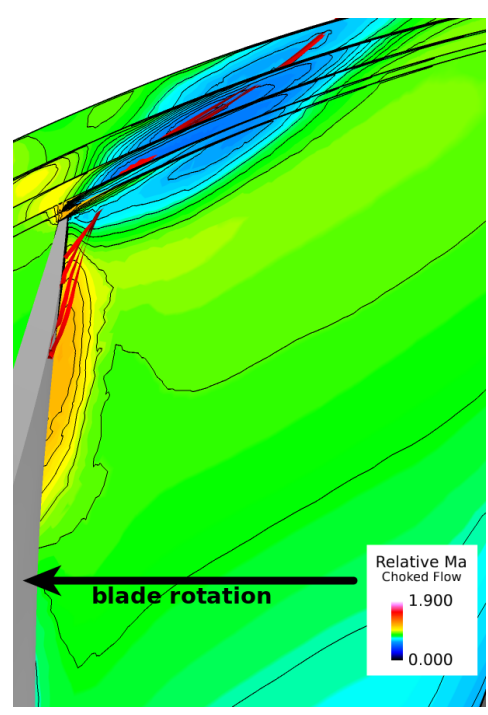

(a) Choked Flow

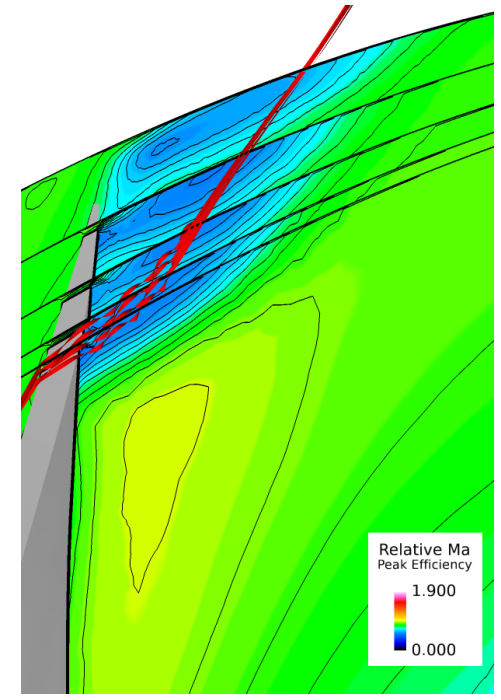

(b) Peak Efficiency

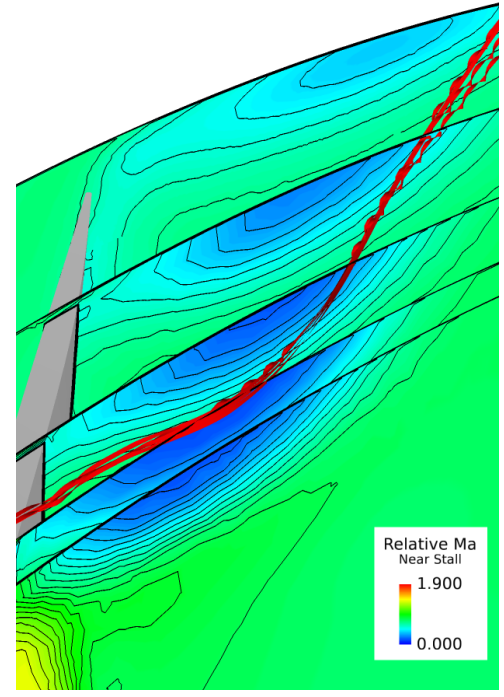

(c) Near Stall

Figure 5.15 Forward-looking-aft view of constant x planes near low Mach number region. 


\subsubsection{Instability Region in Near-Stall Operating Condition}

Next, a closer look is taken at the low momentum region at the near stall flow condition, which is achieved by setting the back pressure to $131.7 \mathrm{kPa}$. This is considered the last stable point since increasing the back pressure by $100 \mathrm{~Pa}$ was found to cause the computed mass flow rate to drop. Various unstable flow features found at this condition are expected to be related to the stalling phenomenon in the turbofan.

Figure 5.16 displays radial cuts, colored by relative momentum magnitude, about the location of the low momentum region at near-stall flow conditions. For the purpose of capturing the full extent of this region, the tip of the blades are aligned with the horizontal direction in these images. The lower surface of the cell (away from the casing) can be spotted at $94.6 \%$ span location, while the core is observed to be approximately at $98.5 \%$ span. The radial cut at the fan blade tip does not allow for a full view of the low momentum region anymore (due to the shape of the casing), but it provides a view of a streak of low momentum flow starting at the leading edge of the fan blade. It can be deduced from these images that the bulk of the flow passing through this region originates from the gap at the blade tip. 


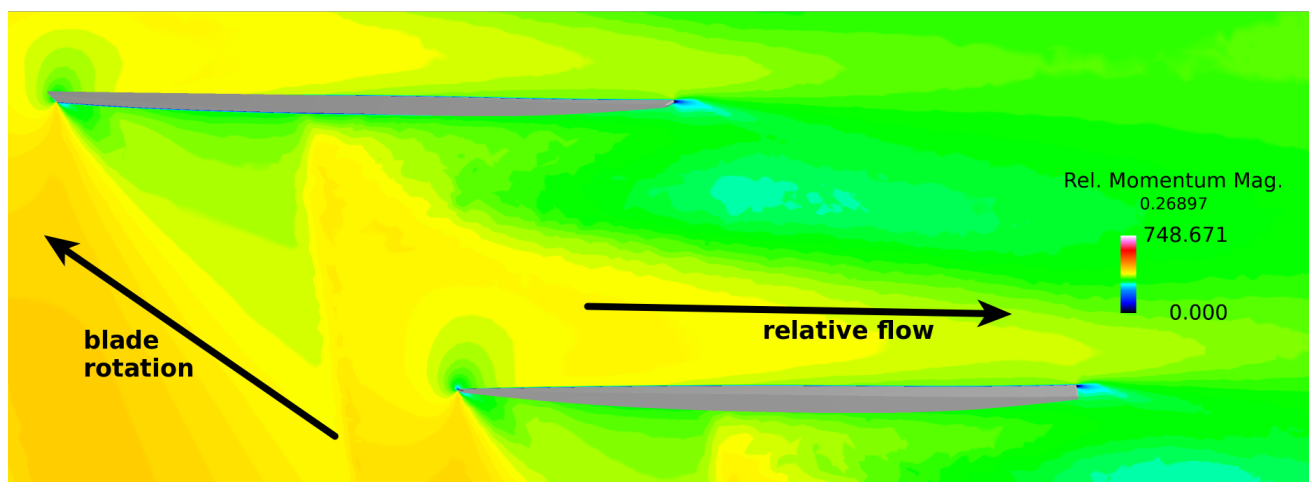

(a) Radial cut at $94.6 \%$ of span.

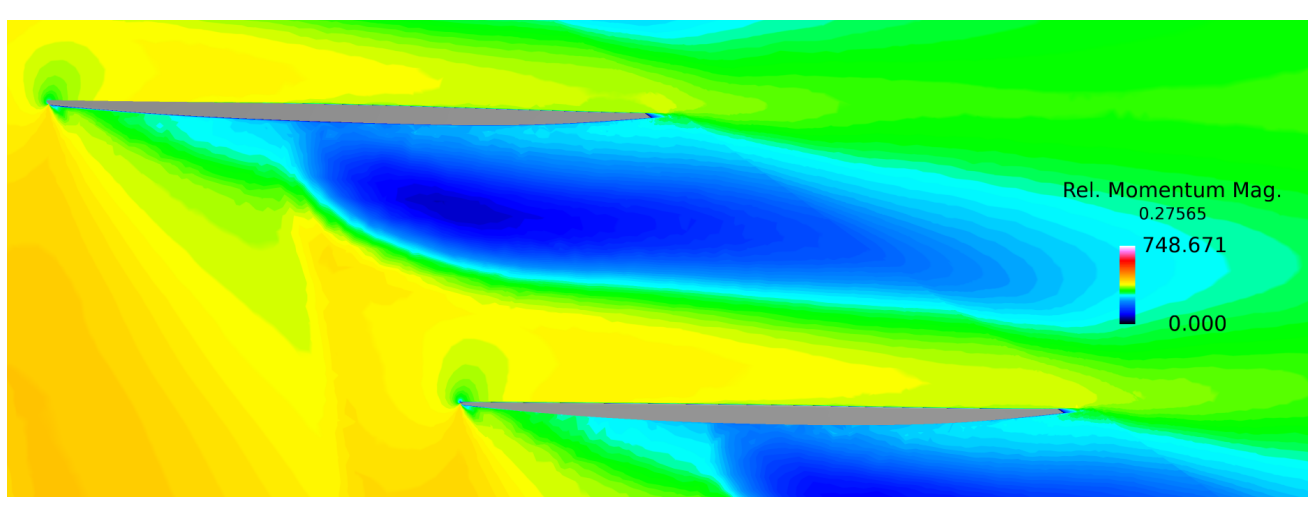

(b) Radial cut at $98.5 \%$ of span.

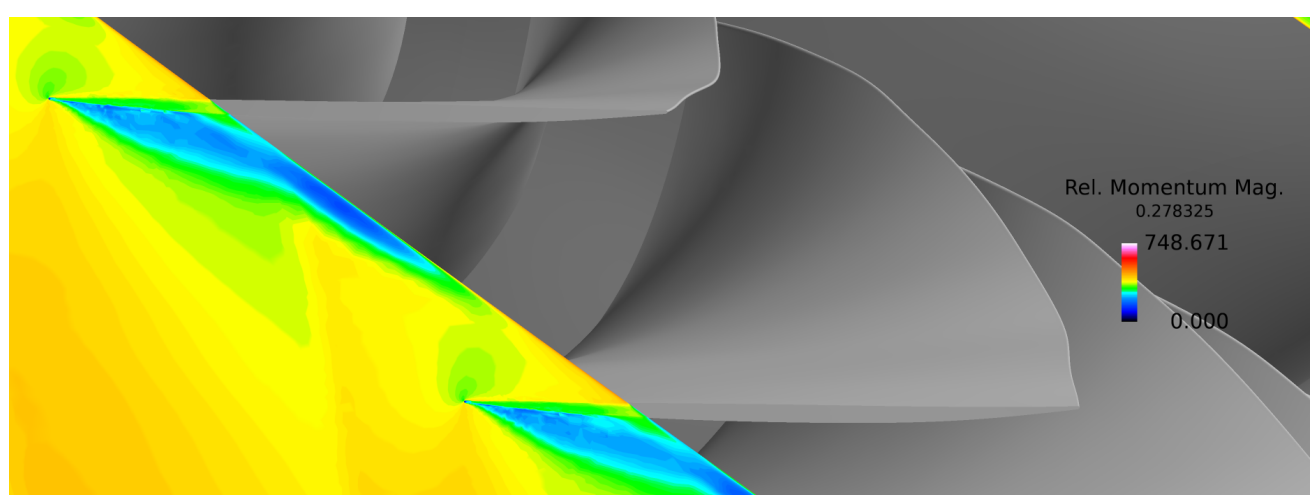

(c) Radial cut at fan tip.

Figure 5.16 Low momentum region near casing for baseline case near stall. 
While Figure 5.16 provides a "top view" of the extent of the low momentum flow region, a view of the region's extent with respect to the passage flow is also desired. In order to achieve this, a plane is defined approximately coincident to the passage shock, called the "shock plane," and various planar cuts are created along the blade tip chord parallel to the shock plane; these are shown in Figure 5.17. The location of the cuts along the blade tip chord are 6\%, 45\% (shock location), $62 \%$ (core of low momentum region), and 100\% (trailing edge). As the cell is a low momentum region, it was expected to create some blockage for the rest of the incoming passage flow. This is more evident through Figure 5.17, which also shows that the flow is forced to accelerate around the cell (Figures 5.17c and 5.17d). 


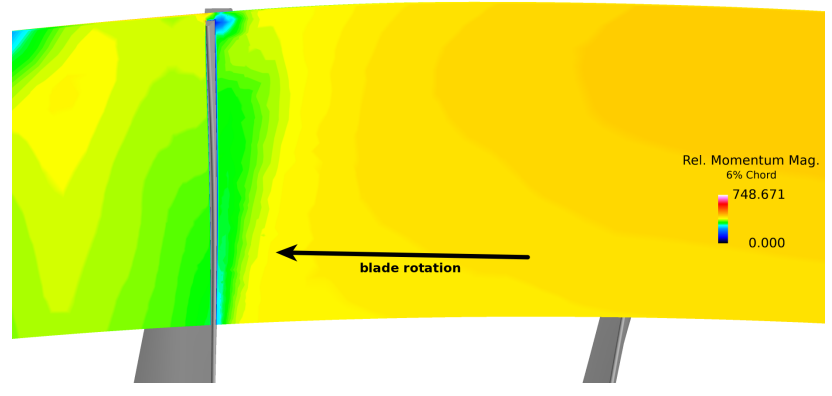

(a) Front tip of low momentum region.

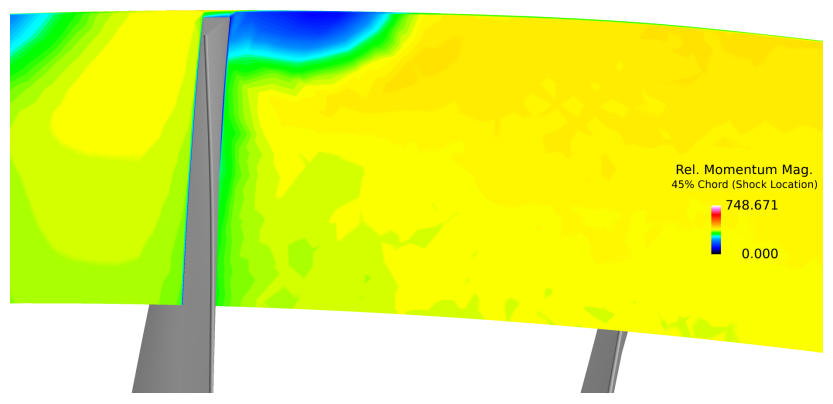

(b) Low momentum region at shock location.

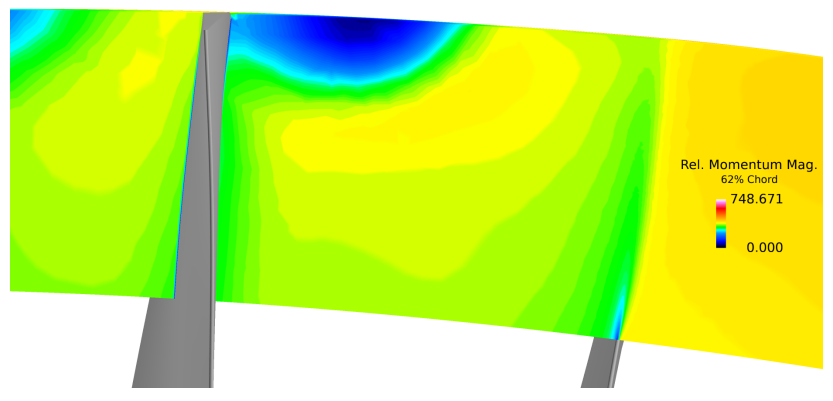

(c) Cross section at core of low momentum region.

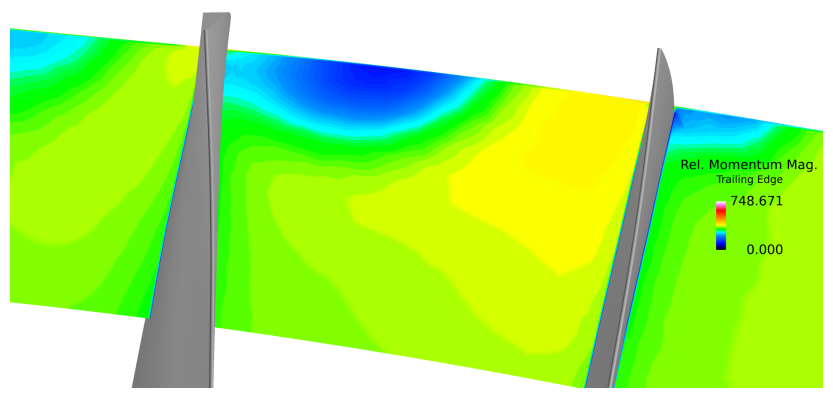

(d) Low momentum region at trailing edge.

Figure 5.17 Forward-looking-aft view of parallel-to-shock plane cuts of low momentum region for baseline case near stall. 
Figure 5.18 provides a three dimensional visualization of the observations made through Figures 5.16 and 5.17. This was achieved by creating an isosurface of the kinematic viscosity $v$ (also known as momentum diffusivity) at a value of $6000 \mathrm{~m}^{2} / \mathrm{s}$. This variable and this value were chosen such that the resulting surface enclosed the region under scrutiny. The isosurface colored by relative Mach number is shown in Figure 5.18a. Figure 5.18b depicts the relative velocity vectors of the flow on the $v$ isosurface. From this view, it is noticed that the fluid changes direction based on its radial and chord location and gives the impression that the fluid in this region possesses some vorticity. In order to further investigate this and observe the path of the flow at various points of the isosurface, ribbon shaped streamlines were seeded and shown in Figure 5.19. Figure 5.19a shows ribbons seeded at three different locations. The seeding of the red ribbons was done on spots of the isosurface showing the lowest values of relative momentum. The path traced shows flow originating at the tip gap location very close to where the shock interacts with the blade. The pink ribbons were seeded on the lowest relative momentum spots of the shock-parallel plane at $62 \%$ of the blade tip chord (location of core cell), and they trace a path of flow crossing the tip gap just upstream the shock. The purple ribbons were seeded along the blade tip from the leading edge to $25 \%$ of the chord and from $65 \%$ of the chord to the trailing edge. These are locations where other ribbons do not cross the tip gap. All streamlines are observed to describe a flow path rolling into a vortex sheet, and the streamlines the cross the tip gap near the shock location describe flow that is more likely to become unstable within the low momentum region. Figure 5.19b shows streamlines that were seeded along the tip gap edges very close to the casing. Compared to the streamlines shown in Figure 5.19a, these also cross the tip gap of the adjacent blade. In addition, the streamlines in Figure 5.19b show that the flow originating from the leading edge to about $40 \%$ 
chord length moves under the flow originating downstream the $40 \%$ chord length location. This behavior is commonly associated with stall flow conditions.

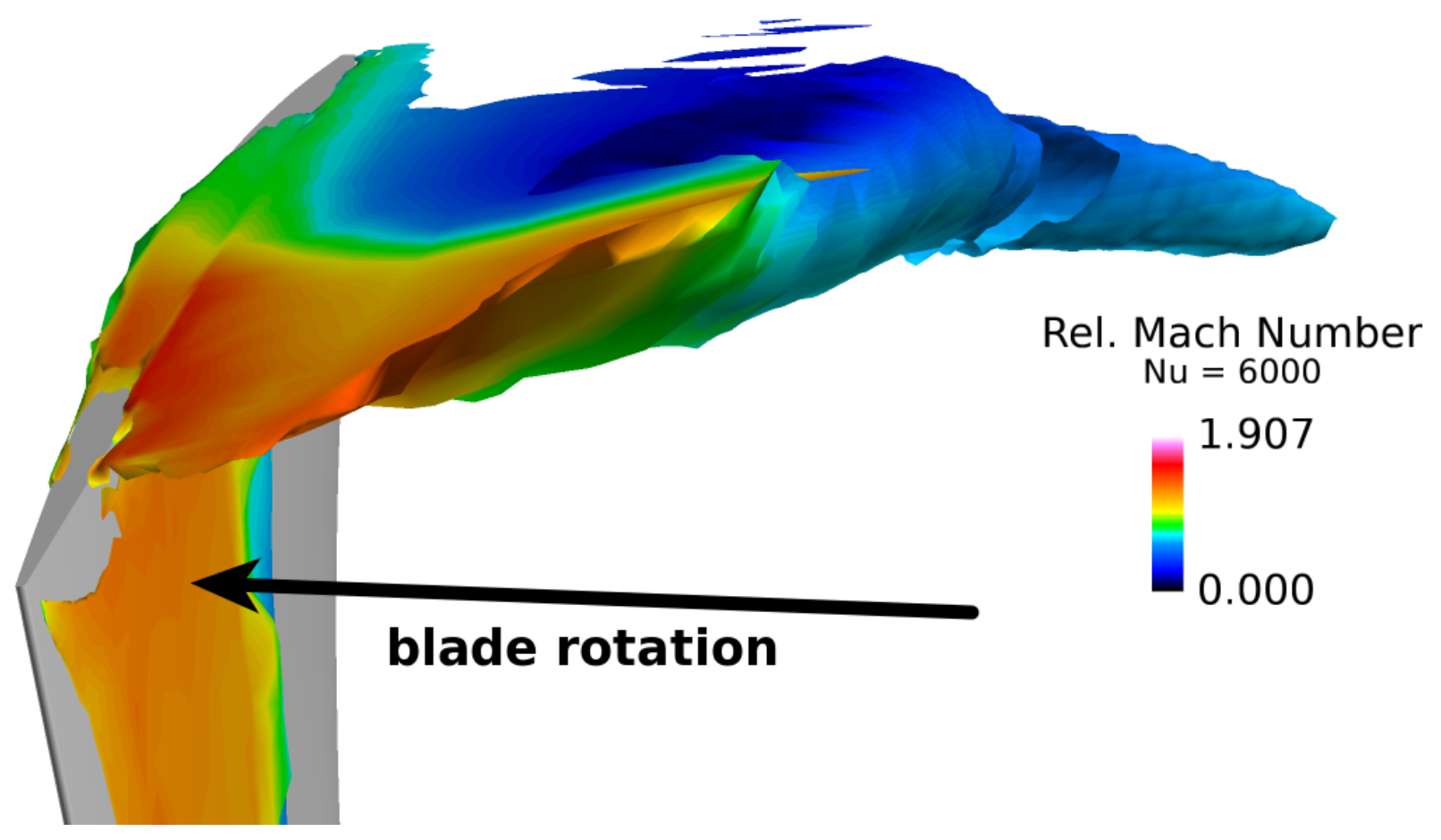

(a) Momentum diffusivity $v$ isosurface. Forward-looking-aft view.

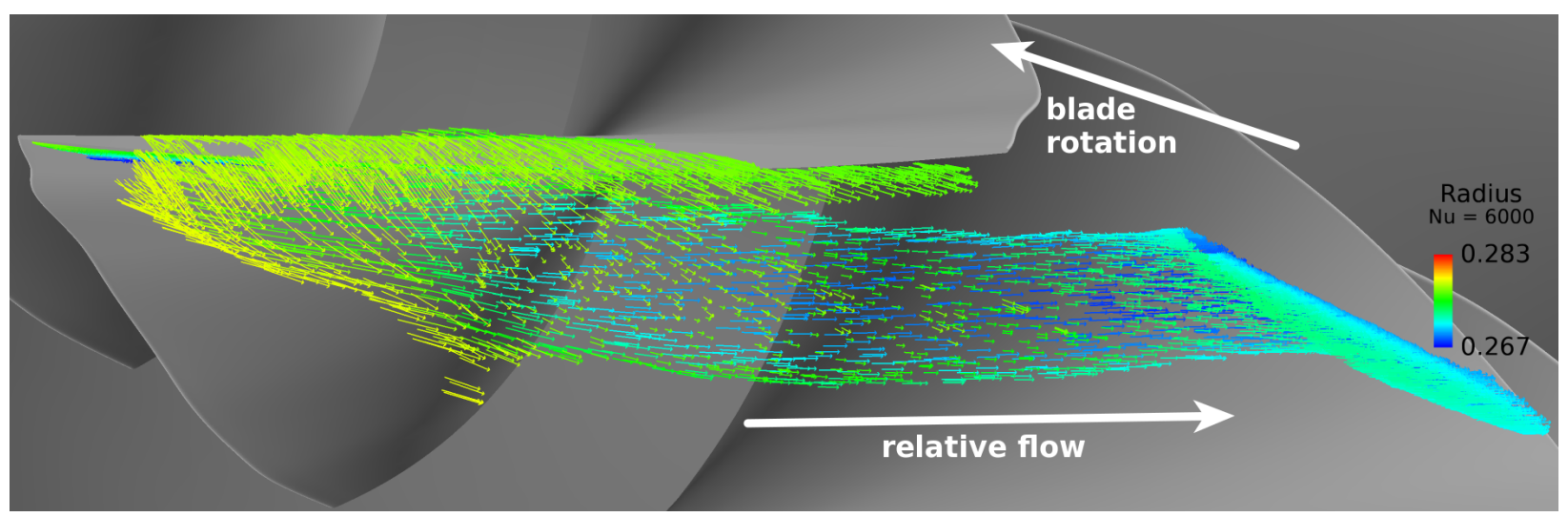

(b) Relative velocity vectors on $v$ isosurface.

Figure 5.18 Low momentum region as exposed by $v$ isosurface. 


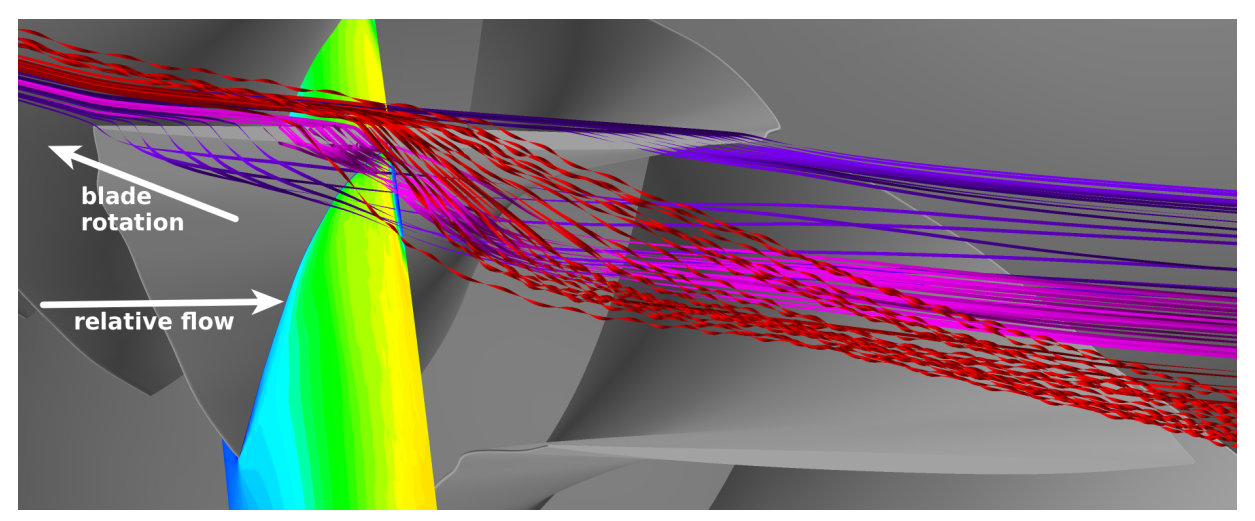

(a) Tip gap flow forming a vortex sheet.

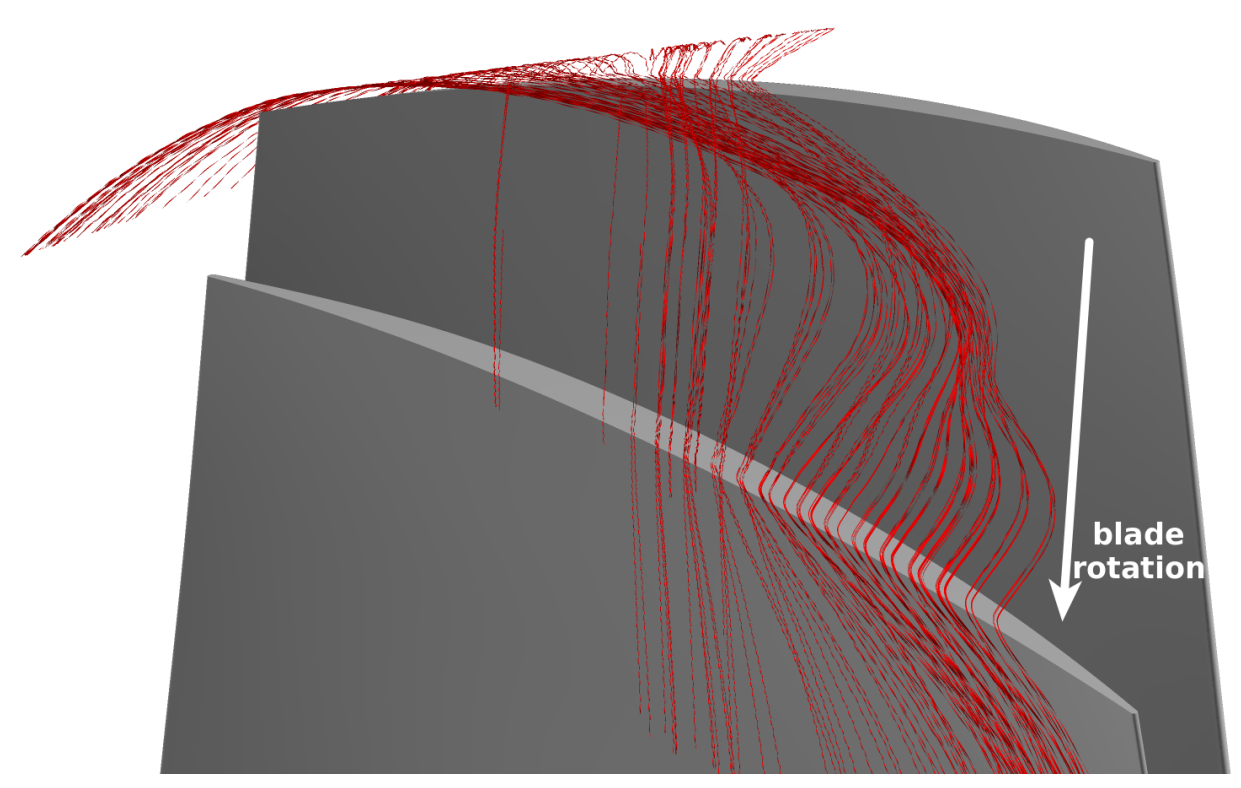

(b) Tip gap flow forming a turning ribbon crossing across flow passages.

Figure 5.19 Tip gap flow patterns for baseline case near stall.

Isosurfaces of the kinematic viscosity $v$ at the same value were created in the stator region, as shown in Figure 5.20. Streamlines were seeded on these isosurfaces in a similar fashion as those in Figure 5.19. No vortices or flow separation are observed from these streamlines as compared to the unstable flow features found in the rotor blade passage. 


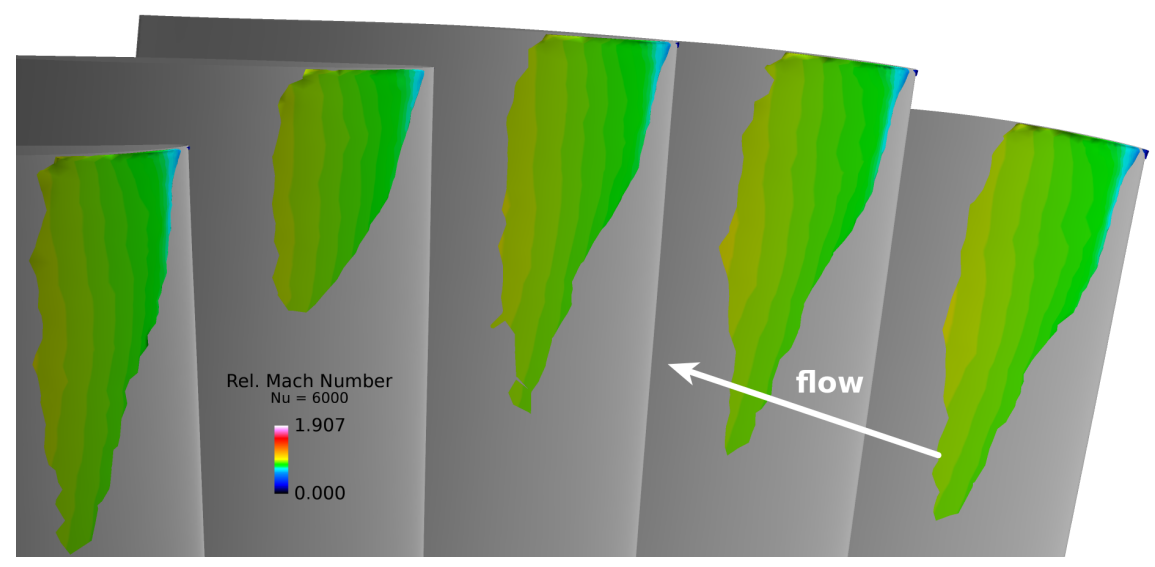

(a) Isosurface of $v$ on stator near casing.

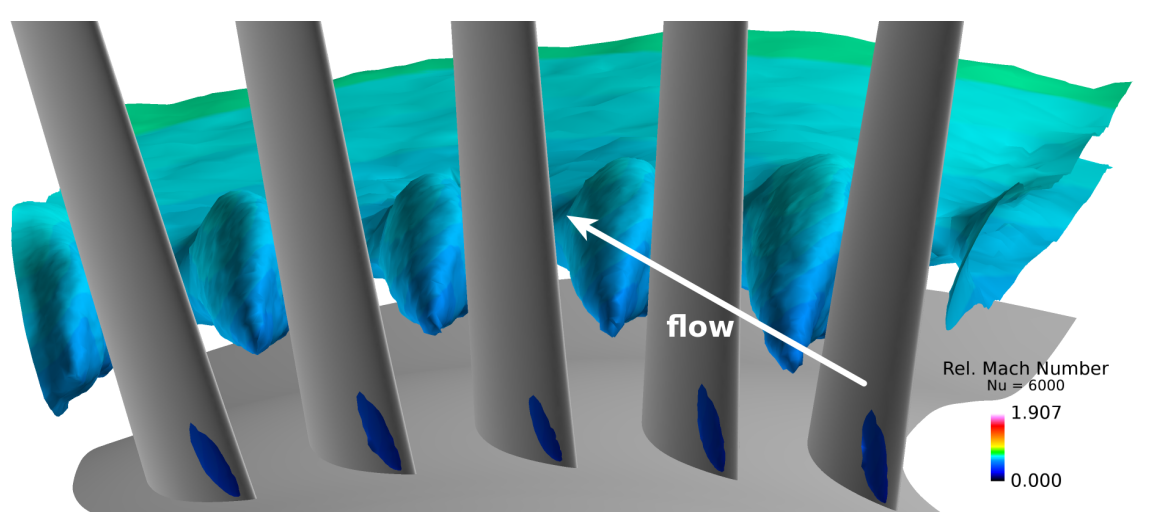

(b) Isosurface of $v$ on stator near hub.

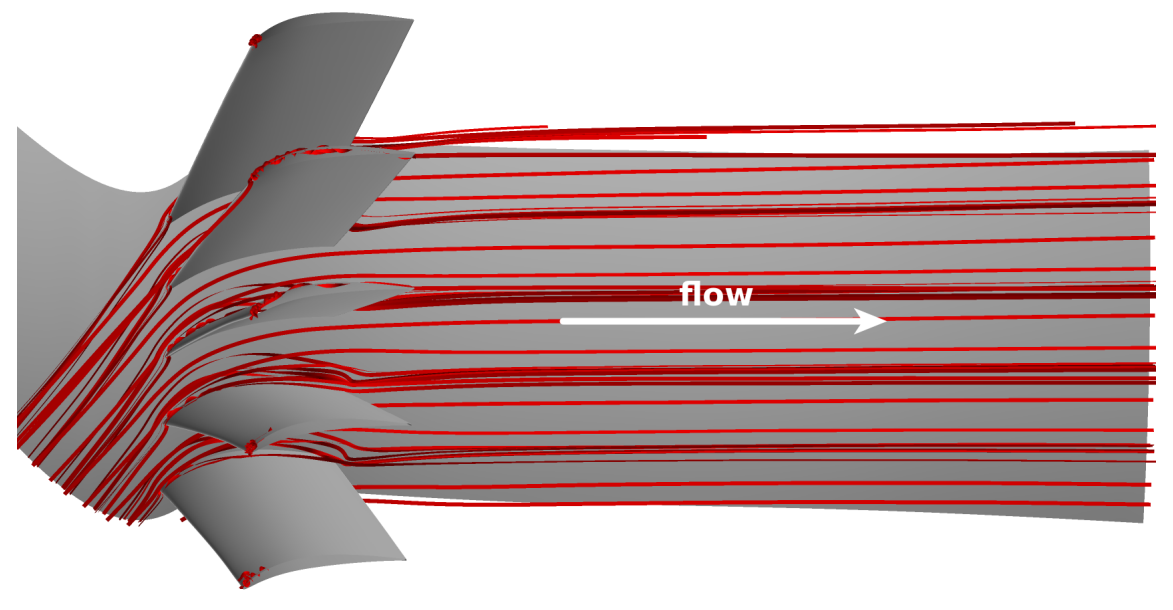

(c) Streamlines on stator.

Figure 5.20 Flow pattern around stator vanes for baseline case near stall. 


\subsection{Influence of the Mesh on the Solutions}

As it was mentioned earlier in Chapter 5, mesh generation can be a very time demanding process, and even more so depending on the complexity of the problem. In this work, almost half of the time and energy spent went toward being able to approximately reproduce the experimental results, and most of that went toward the mesh generation process.

Mesh convergence studies have not been carried in this work, but one such study on the same turbofan model is available from Lin [3]. Although a formal mesh study was not performed to study solution convergence as a function of mesh refinement, various computational meshes (more than 30), different in both the surface mesh and the number of viscous layers, were tested before arriving at solutions that were acceptable.

Stage performance curves obtained from simulations using previous meshes are shown in Figures 5.21 and 5.22. The solutions in Figure 5.21 were obtained with a volume mesh that had a very similar surface mesh to the one used for obtaining the solutions reported in sections 5.1 and 5.2, but it differed in the number of viscous layers. Previously, it was thought that a large number of viscous layers would provide better solutions, and it was decided to insert in the volume mesh as many viscous layers as the meshing software was capable of. That number turned out to be 29 . In contrast, the solutions reported in the previous sections used a mesh with 16 viscous layers. As observed in Figure 5.21, the performance curves obtained using 29 viscous layers (less points on the curve were computed) show a consistent under-prediction of performance metrics. From this it is observed that the solutions can be sensitive to the number of viscous layers and that it is possible to have "too many" viscous layers in the volume mesh. 


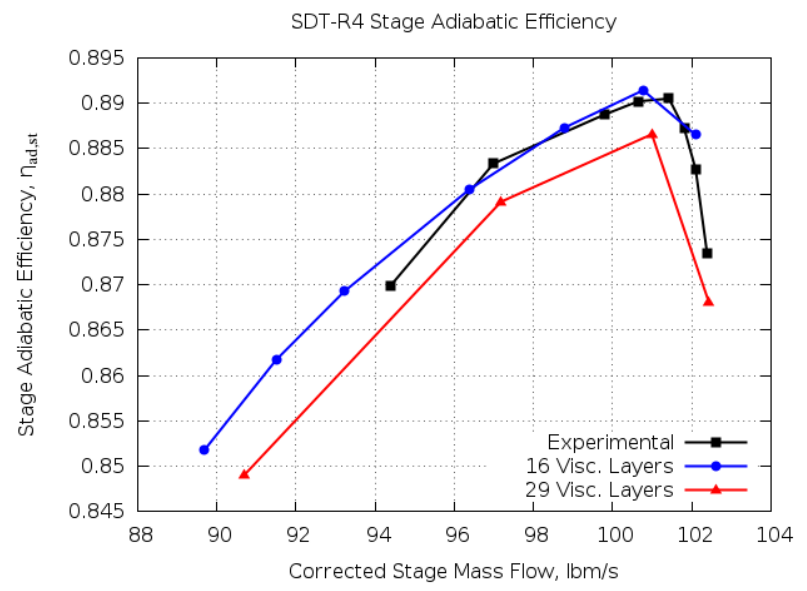

(a) Adiabatic Efficiency

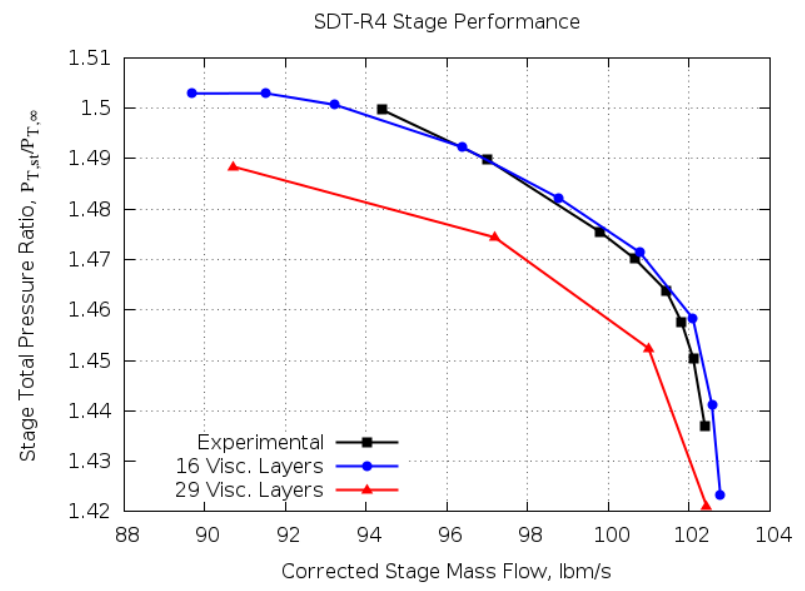

(b) Total Pressure Ratio

Figure 5.21 Comparing performance maps obtained when using a maximum of 29 viscous layers in the computational mesh.

Earlier, a similar approach of using as many as possible viscous layers was used on different surface meshes. The earlier surface meshes were different from the ones used for the solutions reported in the previous sections of this chapter in the clustering of points along the edges of the blades. Previously, significantly less points were used in such edges, with the intention of keeping the mesh point count low in order to use less cores when running the simulation. A similar approach was taken for the clustering of points on the rest of the surfaces away from the edges. The results of using one such mesh are presented in Figure 5.22. This figure includes computed maps from simulations with studs that were carried early in this work. It is observed that, not only were the performance metrics under-predicted, but also the performance curves were shifted left from where the experimentally obtained performance curves stand. From these figures, one thing is observed that is generally known in the field of CFD application for turbo-machinery: the solutions can be very sensitive to changes introduced on the mesh. 


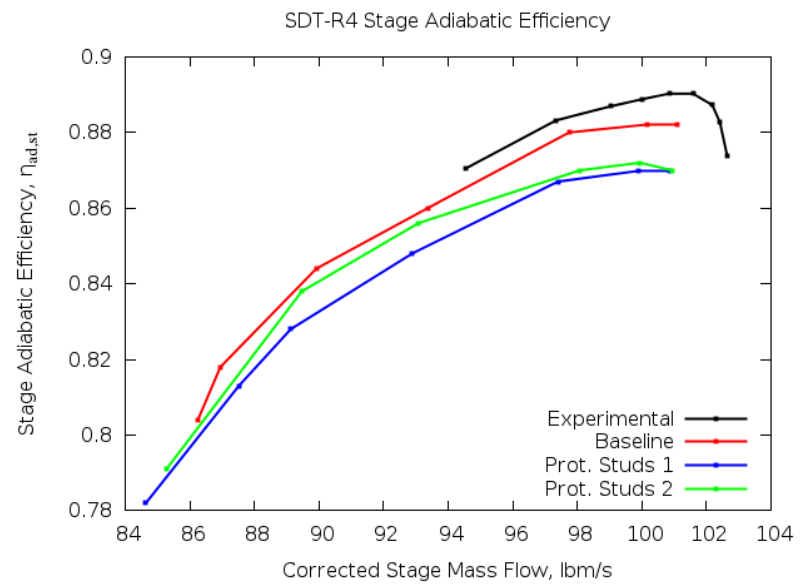

(a) Adiabatic Efficiency

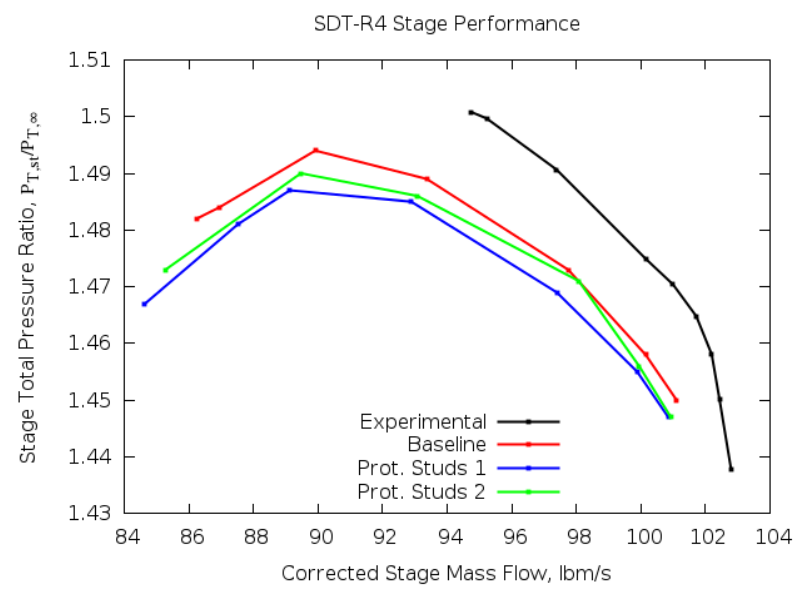

(b) Total Pressure Ratio

Figure 5.22 Performance curves obtained in earlier stages of this work where a maximum of 29 viscous layers and a different surface mesh were used. 


\section{CHAPTER 6}

\section{CASING TREATMENT SIMULATION}

In this chapter, the results of simulating the operation of the turbofan using the protruding studs casing treatment are presented. First, the solutions from using the original studs introduced in subsection 3.2.1.1 are presented through performance maps. Then, performance maps obtained from using the variations of the original studs and the dome shaped studs are given. The chapter finishes with presentation of flow-field features from the effects of the casing treatment through measurement of flow axial velocities and angles at different locations, as well as through visualization of the features using variables such as momentum and Mach number.

Before reporting the results of using the casing treatment, a few notes are useful to mention. First, one of the goals of using any form of stability control is extending the stall margin of the compression system. This means enabling the turbofan or compressor to operate at lower mass flow rates than it could without the stability control. In the performance curves, this effect is manifested as an extension of the curves to the left, with smaller mass flow rates in the operating range as compared to the baseline case (no casing treatment). Second, it is hoped that the stability control extends the operating range with a minimal amount of performance degradation to the compression system. Finally, experimental studies in the field of turbomachinery compare the performance changes due to using stability controls by analyzing flow-fields of different cases with mass flow rates that match. In contrast, this study compares flow-fields by matching conditions of 
back pressure, since the flow conditions are set in the simulations by changing the pressure at the outflow plane. This entails that the cases being compared will be of different mass flow operating conditions. Therefore, this fact will be kept in mind in the presentation of the analyses.

\subsection{Performance Maps}

\subsubsection{Original Studs}

Figures 6.1 and 6.2 present the performance curves of the operation of the turbofan when the original protruding studs are in place. The point of comparison used to evaluate the efficacy of the casing treatment is the performance curve obtained from simulation of the turbofan without casing treatment. Such results were introduced in section 5.1.2 with a mass flow extent similar to what was presented in the report of the experimental analysis of the stage. In the performance curves below, the entire extent of mass flow obtained through the simulations is included, from the choked flow point to the last operating point for which the computations yielded a "stable" flow-field, i.e. mass flow rate converged for the given back pressure condition.

The pressure ratio curves are shown in Figure 6.1. It is observed that using the initial configuration of the elongated "U" shaped studs does not have a great negative impact in the pressure rise characteristics of the turbofan. As computed, the pressure ratio at $122.5 \mathrm{kPa}$ back pressure decreases by about $0.13 \%$ and $0.08 \%$ for Studs 1 and Studs 2, respectively; and at $131 \mathrm{kPa}$ back pressure, the pressure ratio decrease is approximately $0.25 \%$ and $0.13 \%$ for Studs 1 and Studs 2 , respectively. The pressure ratio curves also show that Studs 1 has a greater negative impact than Studs 2. 


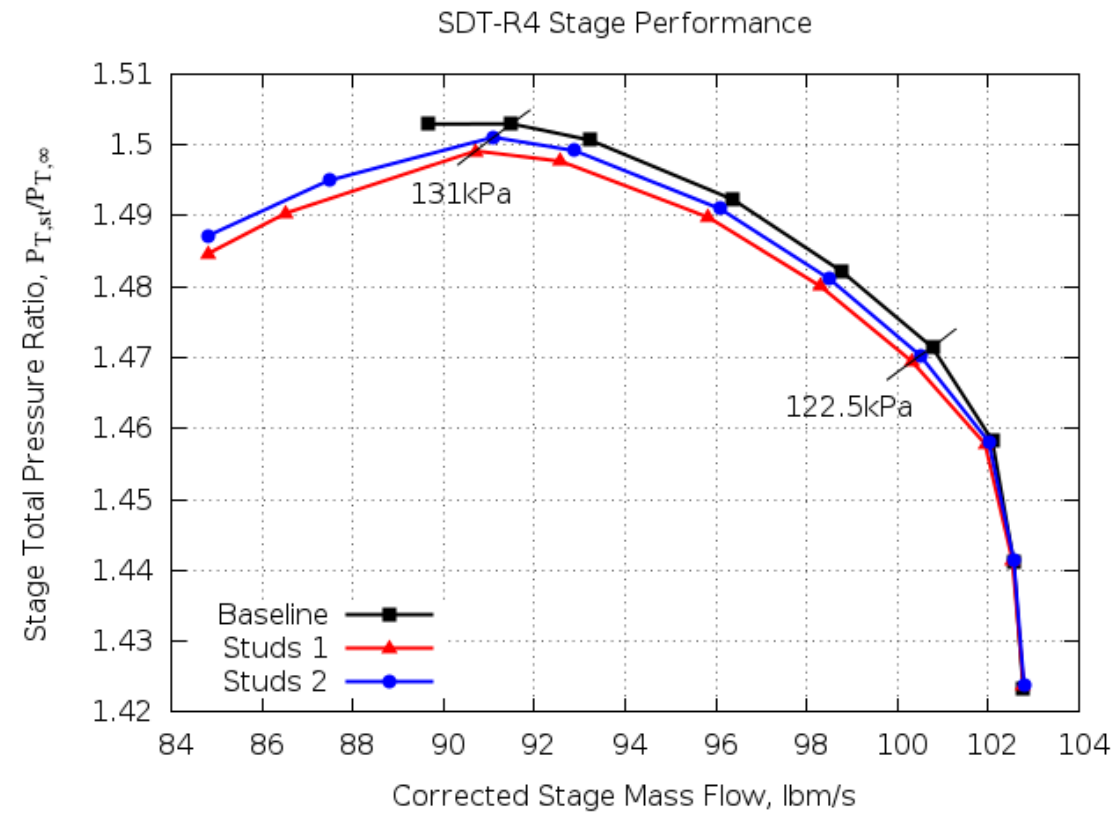

Figure 6.1 Stage total pressure ratio.

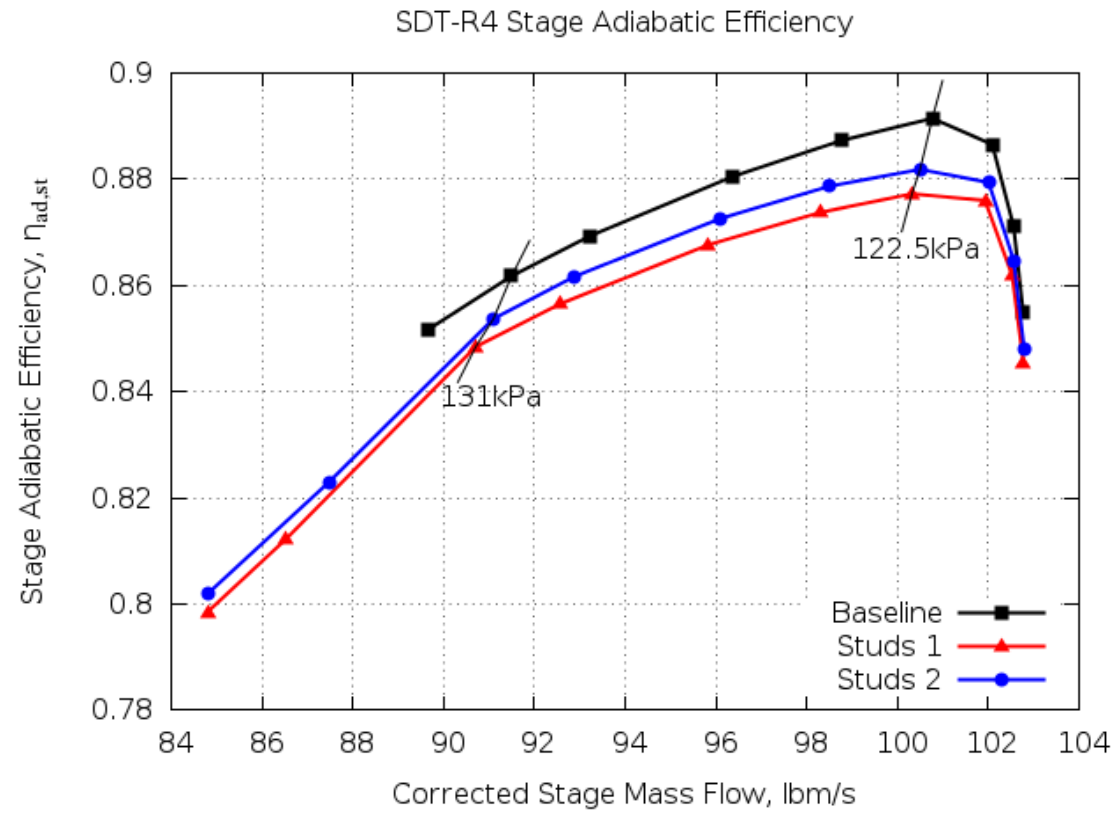

Figure 6.2 Stage total pressure ratio. 


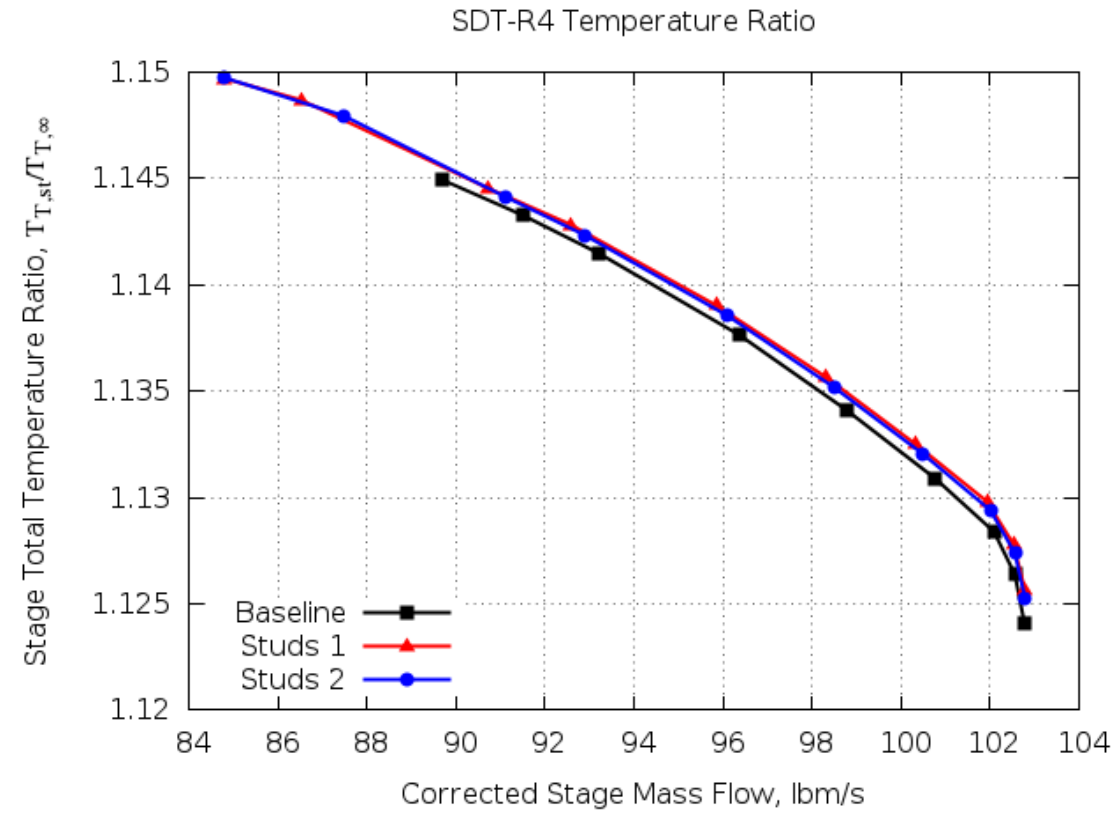

Figure 6.3 Stage total pressure ratio.

As expected, a drop in adiabatic efficiency results from adding protruding structures that interact with the flow. This can be seen from Figure 6.2, which also reveals that Studs 1 has a greater negative impact on efficiency. According to computations, the efficiency drop at a 122.5 $\mathrm{kPa}$ back pressure is about $1.6 \%$ for Studs 1 and $1.1 \%$ for Studs 2; while at $131 \mathrm{kPa}$ back pressure, the efficiencies decrease by about $1.4 \%$ and $0.9 \%$ for Studs 1 and Studs 2 , respectively.

The drop in both efficiency and pressure ratio are credited to the irreversibilities created from the interaction of the flow near the casing with the studs. Such irreversibilites also show as an increase in temperature, as shown in the temperature ratio curves in Figure 6.3. The computations show that the increase in temperature ratio is of about $0.13 \%$, which is mostly consistent throughout the curve and slightly higher for Studs 1. 
The two operating points of comparison up to now have been the back pressure values of $122.5 \mathrm{kPa}$ and $131 \mathrm{kPa}$. This is because the computations yield the highest adiabatic efficiency at $122.5 \mathrm{kPa}$ (Figure 6.2), and because the mass flow difference between the three cases at $131 \mathrm{kPa}$ operating point is less than $1 \%$. Table 6.1 below provides detailed information about the changes.

Table 6.1 Change in performance across results for eleven tested back pressures.

\begin{tabular}{|l|l|l|l|l|l|}
\hline Back Pressure & Case & Flow Rate $(\mathrm{lbm} / \mathrm{s})$ & Pressure Ratio & Temperature Ratio & Adiabatic Efficiency \\
\hline $132.4 \mathrm{kPa}$ & Studs 2 & $84.81(-\%)$ & $1.487(-\%)$ & $1.150(-\%)$ & $0.802(-\%)$ \\
\hline $132.2 \mathrm{kPa}$ & Studs 1 & $84.80(-\%)$ & $1.485(-\%)$ & $1.150(-\%)$ & $0.798(-\%)$ \\
\hline \multirow{3}{*}{$131.7 \mathrm{kPa}$} & Baseline & 89.68 & 1.503 & 1.145 & 0.852 \\
& Studs 1 & $86.53(-3.51 \%)$ & $1.490(-0.84 \%)$ & $1.149(0.33 \%)$ & $0.812(-4.64 \%)$ \\
& Studs 2 & $87.47(-2.46 \%)$ & $1.495(-0.53 \%)$ & $1.148(0.26 \%)$ & $0.823(-3.39 \%)$ \\
\hline \multirow{3}{*}{$131 \mathrm{kPa}$} & Baseline & 91.50 & 1.503 & 1.143 & 0.862 \\
& Studs 1 & $90.71(-0.86 \%)$ & $1.499(-0.25 \%)$ & $1.145(0.11 \%)$ & $0.848(-1.54 \%)$ \\
& Studs 2 & $91.10(-0.44 \%)$ & $1.501(-0.13 \%)$ & $1.144(0.08 \%)$ & $0.854(-0.93 \%)$ \\
\hline \multirow{3}{*}{$130 \mathrm{kPa}$} & Baseline & 93.22 & 1.501 & 1.141 & 0.869 \\
& Studs 1 & $92.58(-0.69 \%)$ & $1.498(-0.20 \%)$ & $1.143(0.12 \%)$ & $0.857(-1.47 \%)$ \\
& Studs 2 & $92.89(-0.35 \%)$ & $1.499(-0.10 \%)$ & $1.142(0.08 \%)$ & $0.862(-0.87 \%)$ \\
\hline \multirow{3}{*}{$127.5 \mathrm{kPa}$} & Baseline & 96.38 & 1.492 & 1.138 & 0.880 \\
& Studs 1 & $95.84(-0.56 \%)$ & $1.490(-0.17 \%)$ & $1.139(0.12 \%)$ & $0.868(-1.46 \%)$ \\
& Studs 2 & $96.09(-0.30 \%)$ & $1.491(-0.09 \%)$ & $1.139(0.08 \%)$ & $0.873(-0.90 \%)$ \\
\hline \multirow{3}{*}{$125 \mathrm{kPa}$} & Baseline & 98.77 & 1.482 & 1.134 & 0.887 \\
& Studs 1 & $98.30(-0.48 \%)$ & $1.480(-0.14 \%)$ & $1.136(0.14 \%)$ & $0.874(-1.53 \%)$ \\
& Studs 2 & $98.51(-0.26 \%)$ & $1.481(-0.07 \%)$ & $1.135(0.09 \%)$ & $0.879(-0.97 \%)$ \\
\hline \multirow{3}{*}{$122.5 \mathrm{kPa}$} & Baseline & 100.77 & 1.471 & 1.131 & 0.891 \\
& Studs 1 & $100.32(-0.45 \%)$ & $1.469(-0.13 \%)$ & $1.133(0.14 \%)$ & $0.877(-1.59 \%)$ \\
& Studs 2 & $100.50(-0.27 \%)$ & $1.470(-0.08 \%)$ & $1.132(0.10 \%)$ & $0.882(-1.08 \%)$ \\
\hline \multirow{3}{*}{$120 \mathrm{kPa}$} & Baseline & 102.09 & 1.458 & 1.128 & 0.887 \\
& Studs 1 & $101.93(-0.16 \%)$ & $1.458(-0.04 \%)$ & $1.130(0.13 \%)$ & $0.876(-1.19 \%)$ \\
& Studs 2 & $102.01(-0.08 \%)$ & $1.458(-0.01 \%)$ & $1.129(0.09 \%)$ & $0.879(-0.81 \%)$ \\
\hline \multirow{3}{*}{$117.5 \mathrm{kPa} \mathrm{kPa}$} & Baseline & 102.56 & 1.441 & 1.126 & 0.871 \\
& Studs 1 & $102.53(-0.03 \%)$ & $1.442(0.02 \%)$ & $1.128(0.13 \%)$ & $0.862(-1.06 \%)$ \\
& Studs 2 & $102.56(0.00 \%)$ & $1.442(0.02 \%)$ & $1.127(0.09 \%)$ & $0.865(-0.73 \%)$ \\
\hline & Baseline & 102.77 & 1.423 & 1.124 & 0.855 \\
& Studs 1 & $102.78(0.01 \%)$ & $1.424(0.04 \%)$ & $1.126(0.14 \%)$ & $0.845(-1.14 \%)$ \\
& Studs 2 & $102.79(0.02 \%)$ & $1.424(0.03 \%)$ & $1.125(0.10 \%)$ & $0.848(-0.80 \%)$ \\
\hline
\end{tabular}


The most important results that the computed performance maps reveal are the computed stall margin extension and the fact that the negative impact on performance is not high. As it was pointed at the beginning of this chapter, the main goal of a stability control system is range extension and, according to the computations, using protruding studs as a form of casing treatment has the potential of achieving this. By mass flow comparison of the last stable operation points (left-most points in the performance curves), a range extension of approximately $5.43 \%$ is observed with respect to mass flow rate for both forms of casing treatment. A slight difference in the efficiency curves suggest that Studs 2 is a more efficient configuration for the studs. The positive aspect of this is that, as suggested by the simulations, this form of casing treatment does not seem to be detrimental on the performance (reduction in mass flow rate, left shifting the entire curve), and that it can be used effectively when the compression system operates at off-design conditions. From this reasoning, it can be said that the studs could be introduced in the flow field only when it is detected that the flow-field approaches the unstable region, and they could be removed or retracted when normal operating conditions are re-established.

\subsubsection{Variation of Studs}

After assessing the performance changes introduced by the protruding studs, it was decided to test the performance of variations of the structures by dimension and also by shape. The purpose is to define possible directions of exploration of new stud geometries. First, different levels of protrusion were tested without changing other dimensions of the studs. The tested protrusion levels were, with respect to the original studs height, 50\% (HS1 \& HS2), 125\% (LS1 \& LS2), and $150 \%$ (XS1 \& XS2). Then, a set of studs similar to Studs 1 were tested that were half in their 
tangential extension and twice in number (W50). Finally, dome shaped studs were tested. They were generated by half a revolution of the "U" shape, and four times as many as the original studs were located equally spaced. Figure 6.4 gives a graphic description of the variations tested.

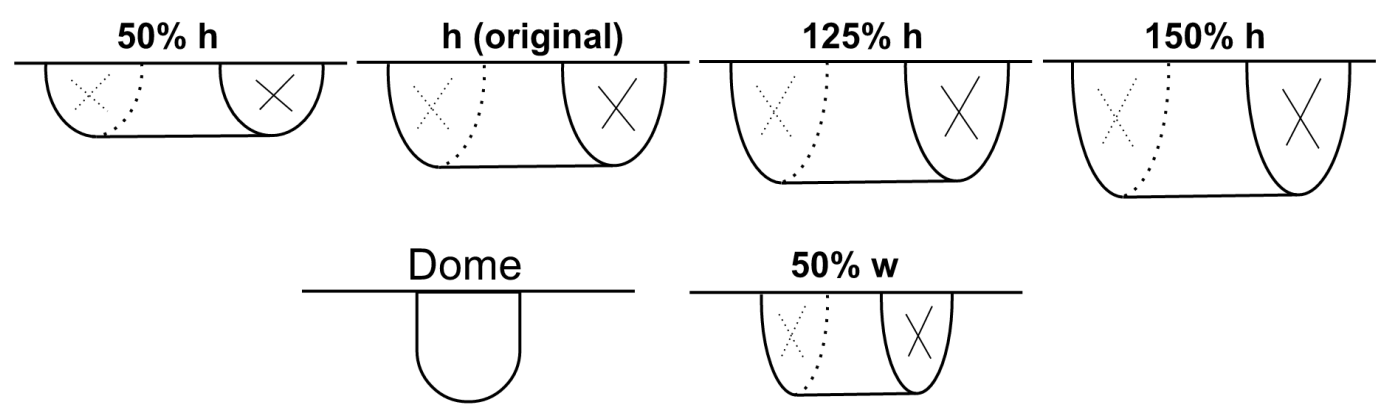

Figure 6.4 New studs tested.

\subsubsection{Performance by Levels of Protrusion}

Figures 6.5 through 6.7 are the performance curves of the studs with changed protrusion levels. The performance curves of the original studs are included for comparison. It is observed that the level of protrusion could be a variable of range extension. The studs with $50 \%$ height of the original studs seem to provide some extension. However, the extension that the $50 \%$ height studs provide is not $50 \%$ of the extension that the original studs provide. The studs with $125 \%$ height of the originals seem to provide a slightly higher extension, which suggests that there may be some room for exploration in terms of levels of protrusion for even greater range extension. However, there is a point at which increasing protrusion further could diminish the extension gains, as observed from the performance curves of the studs with $150 \%$ height of the originals. This combined with the degradation in the performance of the turbofan make using this level of protrusion completely counterproductive. In contrast to range extension, the decrease in pressure 
ratio and adiabatic efficiency seems to vary more directly with protrusion level. Finally, it is also observed that the studs with sides aligned with the rotor blade stagger angle, at any level of protrusion, have a greater detrimental effect on pressure ratio and efficiency.

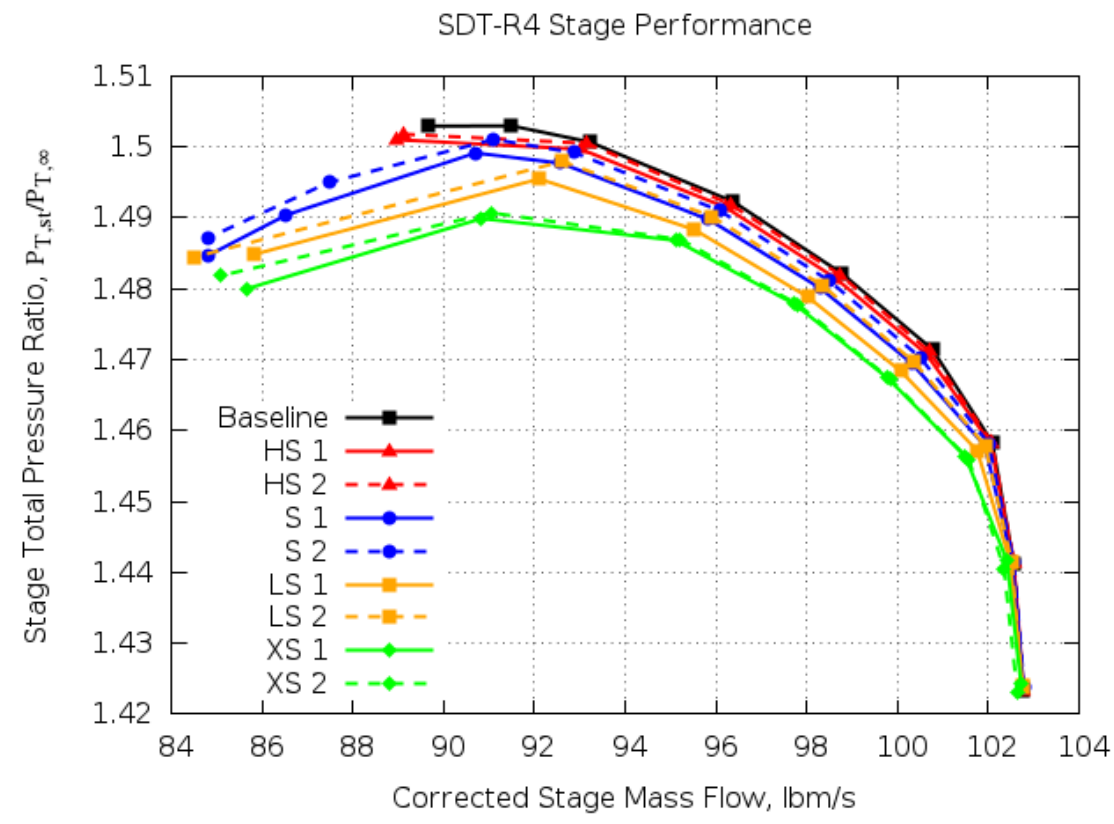

Figure 6.5 Stage total pressure ratio. 


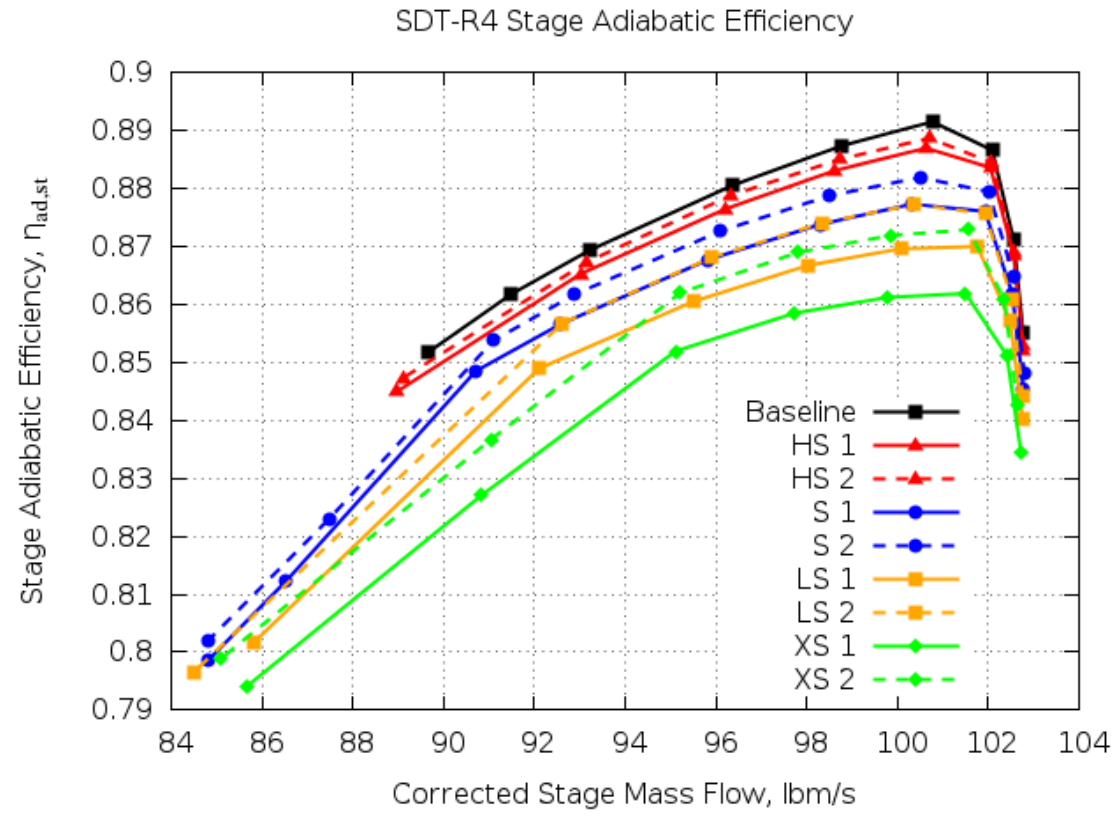

Figure 6.6 Stage adiabatic efficiency.

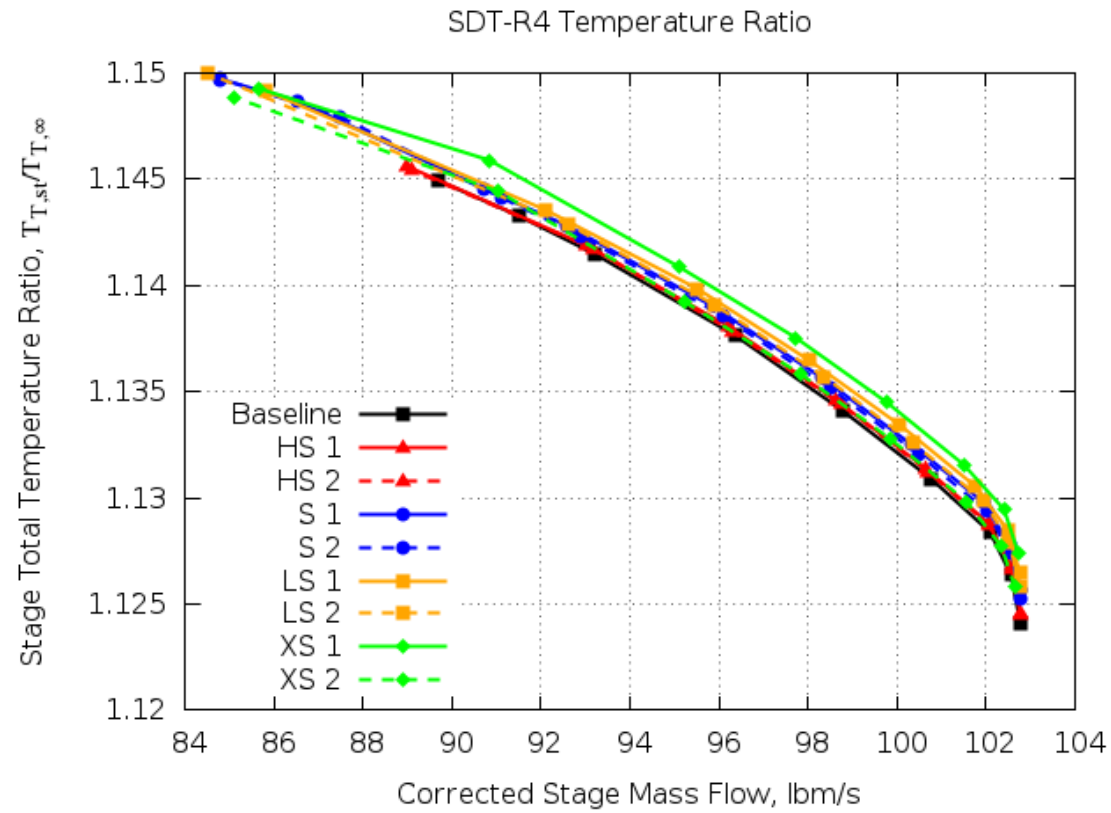

Figure 6.7 Stage total temperature ratio. 


\subsubsection{Half Extension and Dome Shaped Studs}

Figures 6.8 through 6.10 provide the performance curves for the W50 studs, and the dome shaped studs. The performance curve obtained using Studs 2 is included for comparison. It is observed that the W50 studs seem to provide a greater stall margin compared to the dome shaped studs, and that using neither types of studs seem to have a significant loss of efficiency or pressure rise. However, the computed increase in stall margin is much less than that offered by Studs 2 .

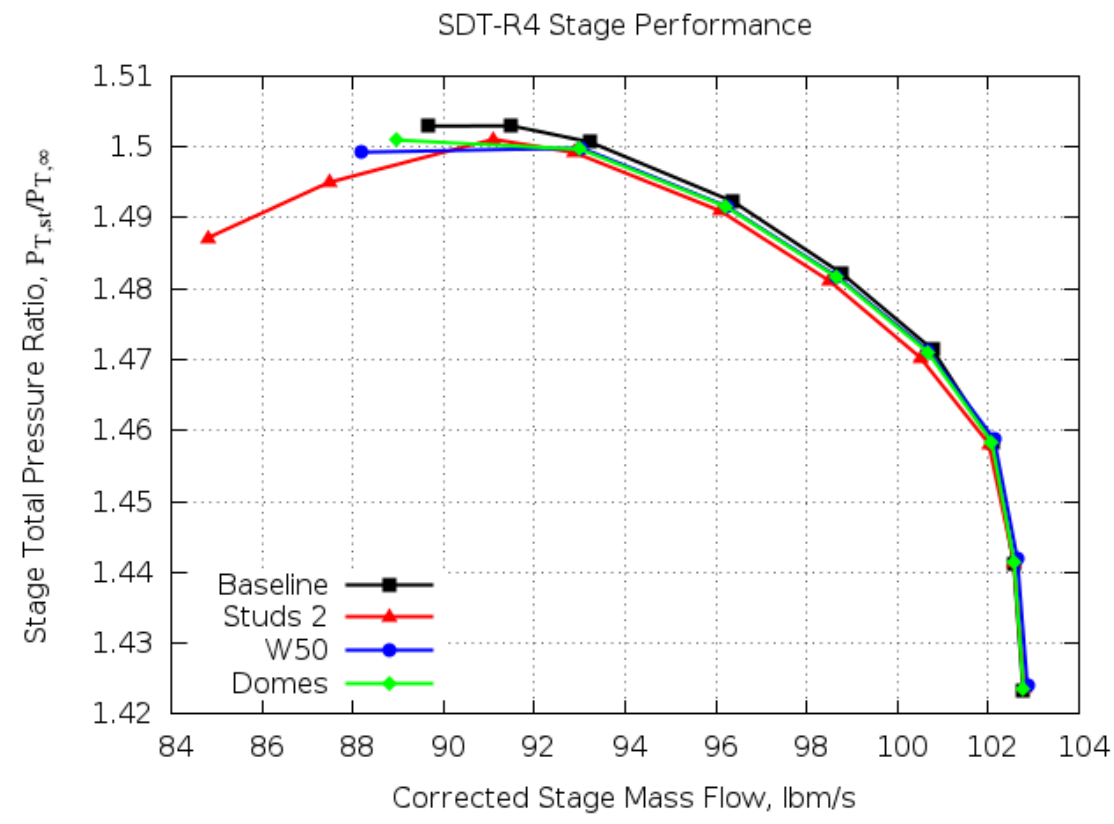

Figure 6.8 Stage total pressure ratio. 


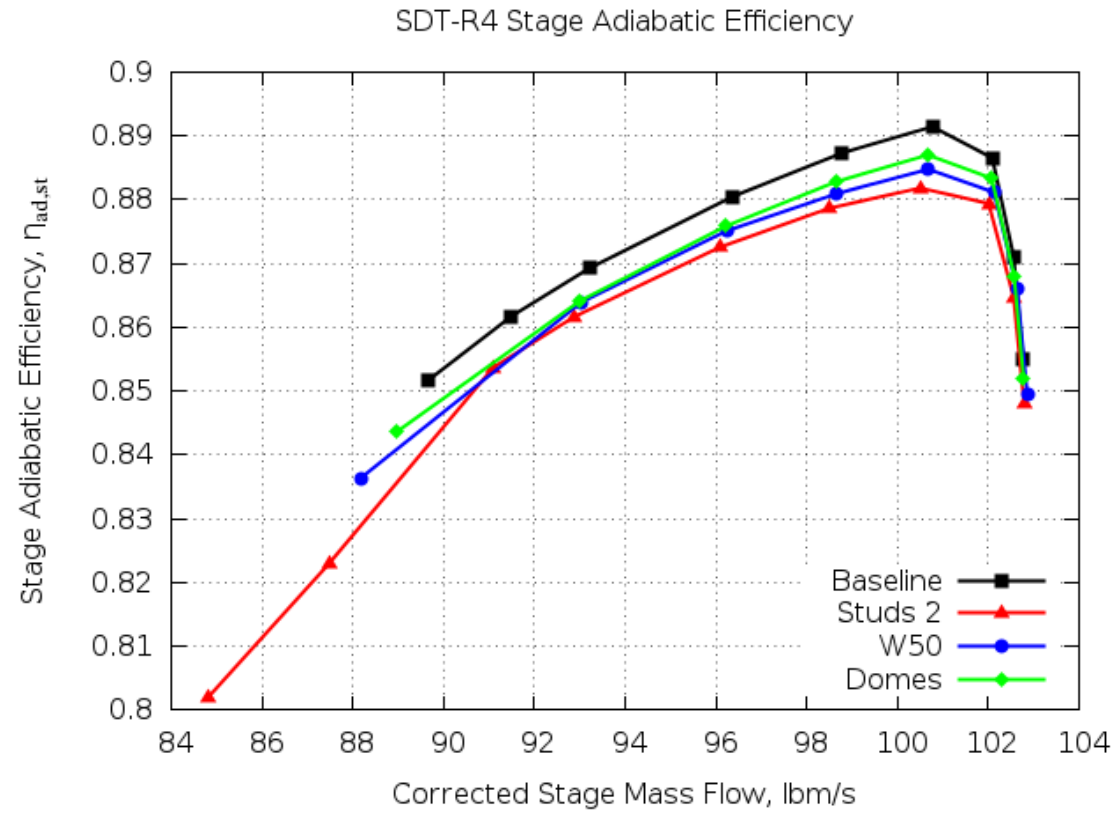

Figure 6.9 Stage adiabatic efficiency.

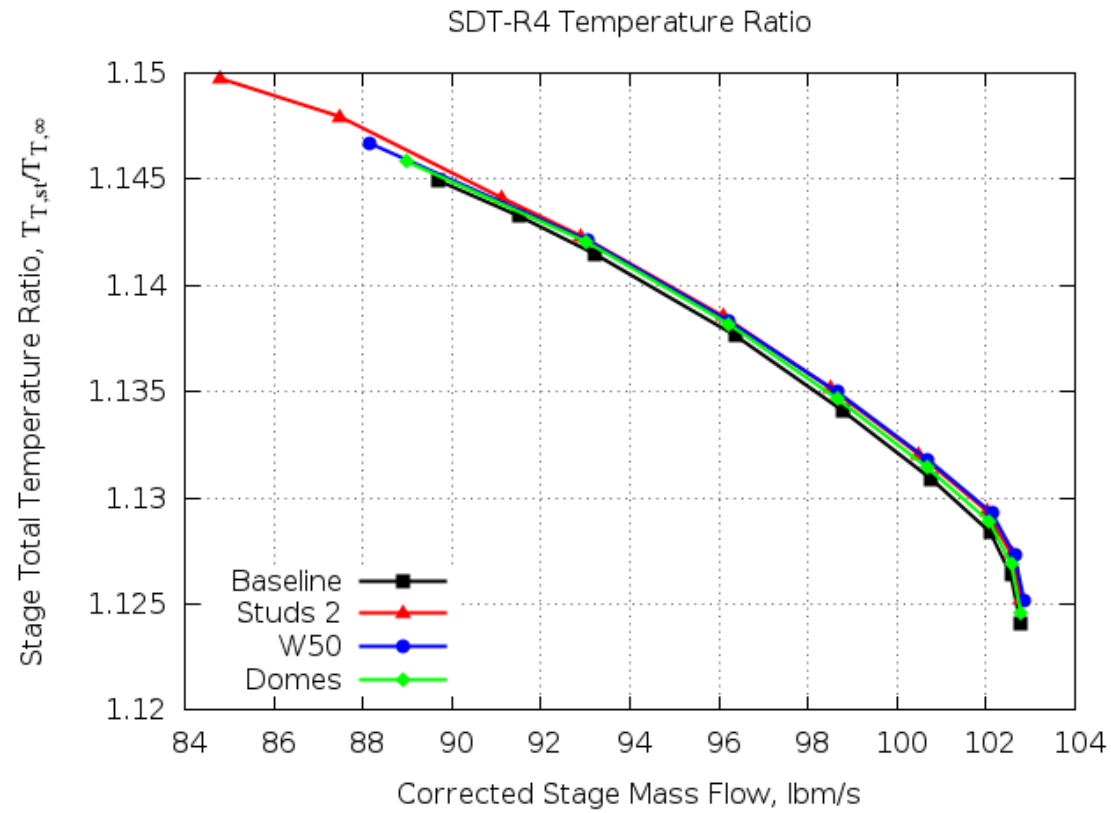

Figure 6.10 Stage total temperature ratio. 


\subsection{Flow-Field Analysis}

In this section, features of the flowfield that are believed to be important in the stability of the stage are explored. Measures of axial velocities and turning angles are explored upstream and downstream of the fan as initial steps in understanding general characteristics of the flow. Next, visualizations of isosurfaces and streamlines are provided that give insight on what certain flow phenomena may look like physically. Finally, similar visualizations of the flow around the studs are given in an attempt to explain the differences in performance between the two original stud configurations. The analysis in this section is provided only for the baseline case and the original studs. The flow conditions explored are set by back pressures of $122.5 \mathrm{kPa}$ (highest computed efficiency), $131 \mathrm{kPa}$ (highest computed pressure ratio), $131.7 \mathrm{kPa}$ (near stall condition for baseline case), and $132.2 \mathrm{kPa}$ for Studs 1 and $132.4 \mathrm{kPa}$ for Studs 2 (the new near stall conditions using casing treatment).

\subsubsection{Radial Distribution of Axial Flow Velocity}

In order to assess any changes in the flow-field away from the casing, the radial distribution of axial velocities was taken for solutions at conditions of $122.5 \mathrm{kPa}, 131 \mathrm{kPa}$ and $131.7 \mathrm{kPa}$ back pressures. Visualizations of the axial momentum component for the $131 \mathrm{kPa}$ and $131.7 \mathrm{kPa}$ cases are available to correlate with the radial distribution plots. The radial measurements were taken at planes in two axial locations, upstream and downstream of the fan blades. The location of the upstream plane is one axial chord length forward of the fan leading edge tip, and the location of the downstream plane is one axial chord length aft of the fan trailing edge tip. The location of these sampling planes in the stage were chosen far enough from the fan in order to avoid sampling in a 
region that is strongly influenced by the presence of the fan. They are shown in Figure 6.11. The span at the planes is defined as the radial location from the hub to the casing.

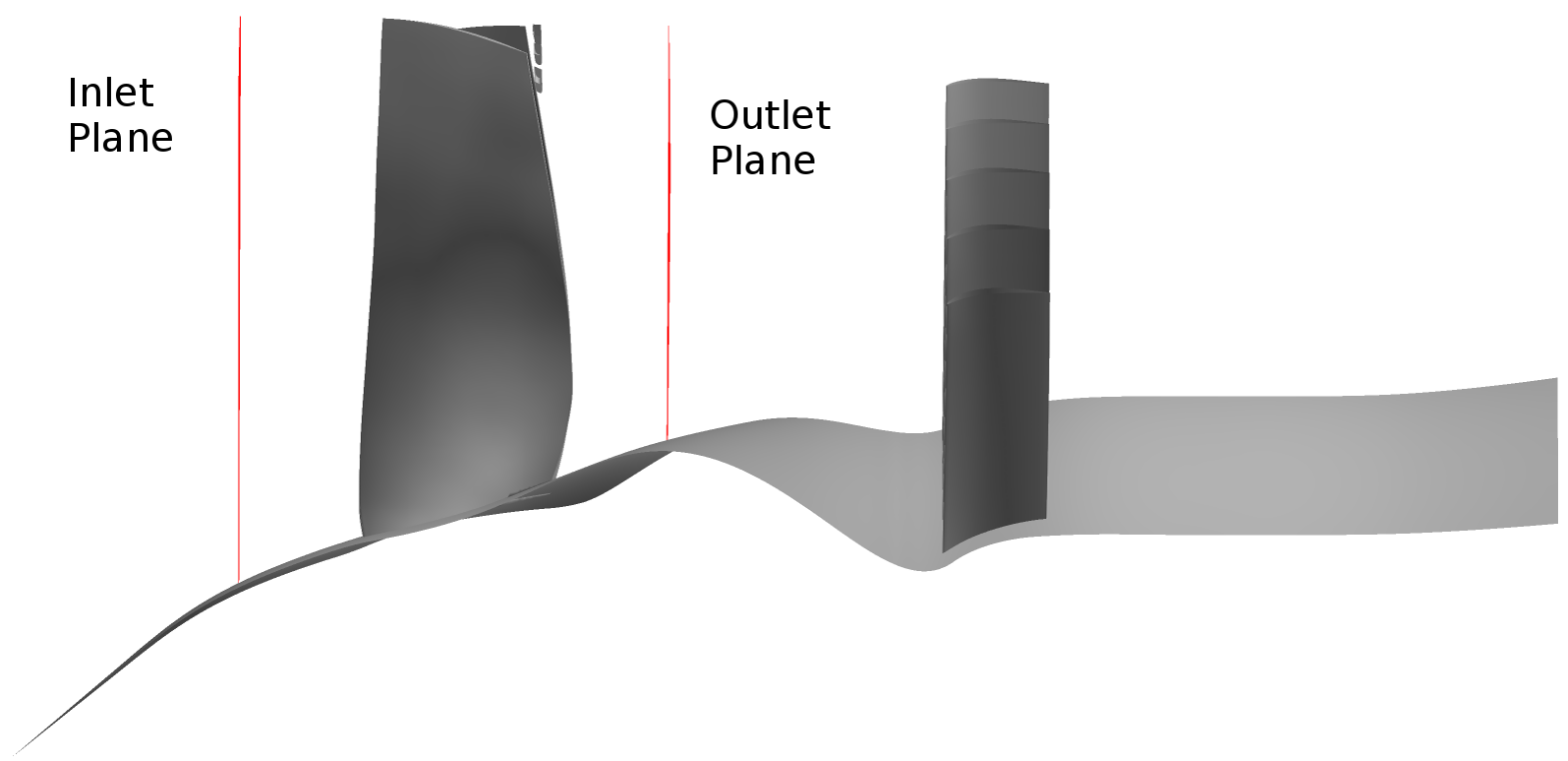

Figure 6.11 Axial location of planes, where radial distribution samples were taken, displaying studs near the trailing edge of the rotor blades.

Figure 6.12 presents the radial distribution of the axial velocities for the solutions of the 122.5 kPa back pressure flow conditions. In the plot for the inlet plane measurements, it is observed, for the three cases that from the hub to about $4 \%$ span, the flow quickly picks up to about $90 \%$ of the maximum speed reported in the plot. From $4 \%$ to about $40 \%$ span, the flow increases to about $95 \%$ of the maximum axial speed almost linearly. From then, the flow arrives at its maximum axial speed at $60 \%$ span and decreases to approximately $95 \%$ maximum axial velocity at $85 \%$ span. From about $85 \%$ to $98 \%$ span, the flow decreases to approximately $85 \%$ of maximum axial speed, and then quickly to zero at the casing. Although, the profile described here is very similar for all 
three cases, it is noticed that the profiles corresponding to the casing treatments are shifted to the left slightly. This can be attributed to the fact that protruding structures are imposing a certain blockage downstream and less fluid is now moving through the duct.

In the case of the axial velocities at the outlet plane, it is observed that the distribution is similar for the three cases. Starting at the hub to $7 \%$ span, the flow goes from zero axial velocity to about $90 \%$ of the maximum. From there to about $25 \%$ span, the axial velocity seems to remain relatively unchanged or even decrease slightly. Then, up to $60 \%$ span location, the axial velocity increases linearly to about $97 \%$ of the maximum. From there to $85 \%$ or $90 \%$ span, the flow increases to maximum axial velocity. From $90 \%$ span to the endwall, the flow goes from maximum axial speed to zero. Compared to the baseline, the casing treatment profile is shifted forward (to the right), and the velocity transition from maximum to zero happens more quickly. This shift of the profiles signals the effect of flow being displaced away from the casing toward regions of higher flow velocity. 
Radial Distribution of Axial Velocity - $122.5 \mathrm{kPa}$
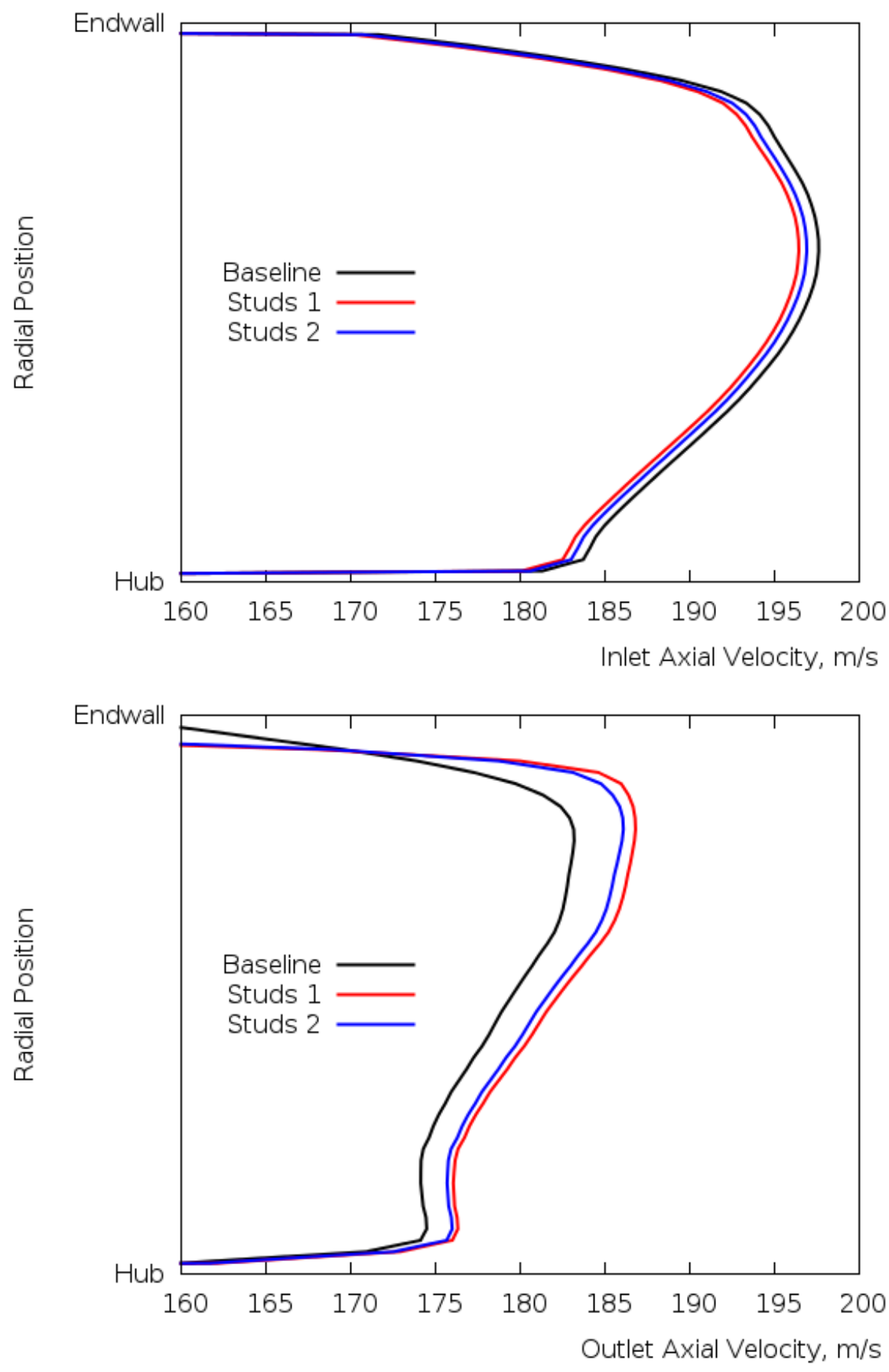

Figure 6.12 Radial distribution of axial velocities at computed peak efficiency. 
Figure 6.13 presents the radial distribution of the axial velocity component for the $131 \mathrm{kPa}$ back pressure operating condition. The profiles of the distributions have remained similar to the ones for the $122.5 \mathrm{kPa}$ operating point except for two important differences. The first is that the axial velocities have reduced at both inlet and outlet planes relative to those in the $122.5 \mathrm{kPa}$ case. This correlates in part to the fact that the mass flow rate is less at this condition. The second difference is that, at the outlet plane, the radial location of the maximum axial speed has shifted down to $60 \%$ span. Once more, the transition from axial speed at $90 \%$ span to zero at the casing is faster in the casing treatment case than in the baseline case. Figure 6.14 is provided to go with the axial velocities radial distribution of the $131 \mathrm{kPa}$ operating condition. This figure displays radial cuts colored by axial momentum (flow moving from top to bottom) using a color striped color map. The radial location is $75 \%$ fan blade span (about $78 \%$ span at the outlet sampling plane), which was chosen in order to capture some of the greater differences in axial velocity. By careful observation, it is seen that at this radial location the regions of higher axial momentum are greater in the casing treatment cases than in the baseline case. It can be observed that, in the casing treatment cases, the regions colored in red extend from the bottom into the blade passage region in some locations.

Figures 6.15 and 6.16 are axial velocity radial distribution profiles for the $131.7 \mathrm{kPa}$ operating point and their corresponding axial momentum visualization. The axial velocities across the profile are shown to have further decreased relative to those in the $131 \mathrm{kPa}$ case at both the inlet and outlet planes. Also, at the outlet plane, the casing treatment profiles displays smaller axial velocities than the baseline case, which did not occur for the two operating points shown before. These observations correspond to the fact that the mass flow rate has further decreased in the casing treatment cases. Regardless of the effect of the protruding studs, the amount of flow throughout the 
duct is greater in the baseline case than in the casing treatment cases. This can be visually verified by Figure 6.16 (flow moving from top to bottom) by observing that the red coloring occupies greater regions in the baseline case images. 
Radial Distribution of Axial Velocity - $131 \mathrm{kPa}$
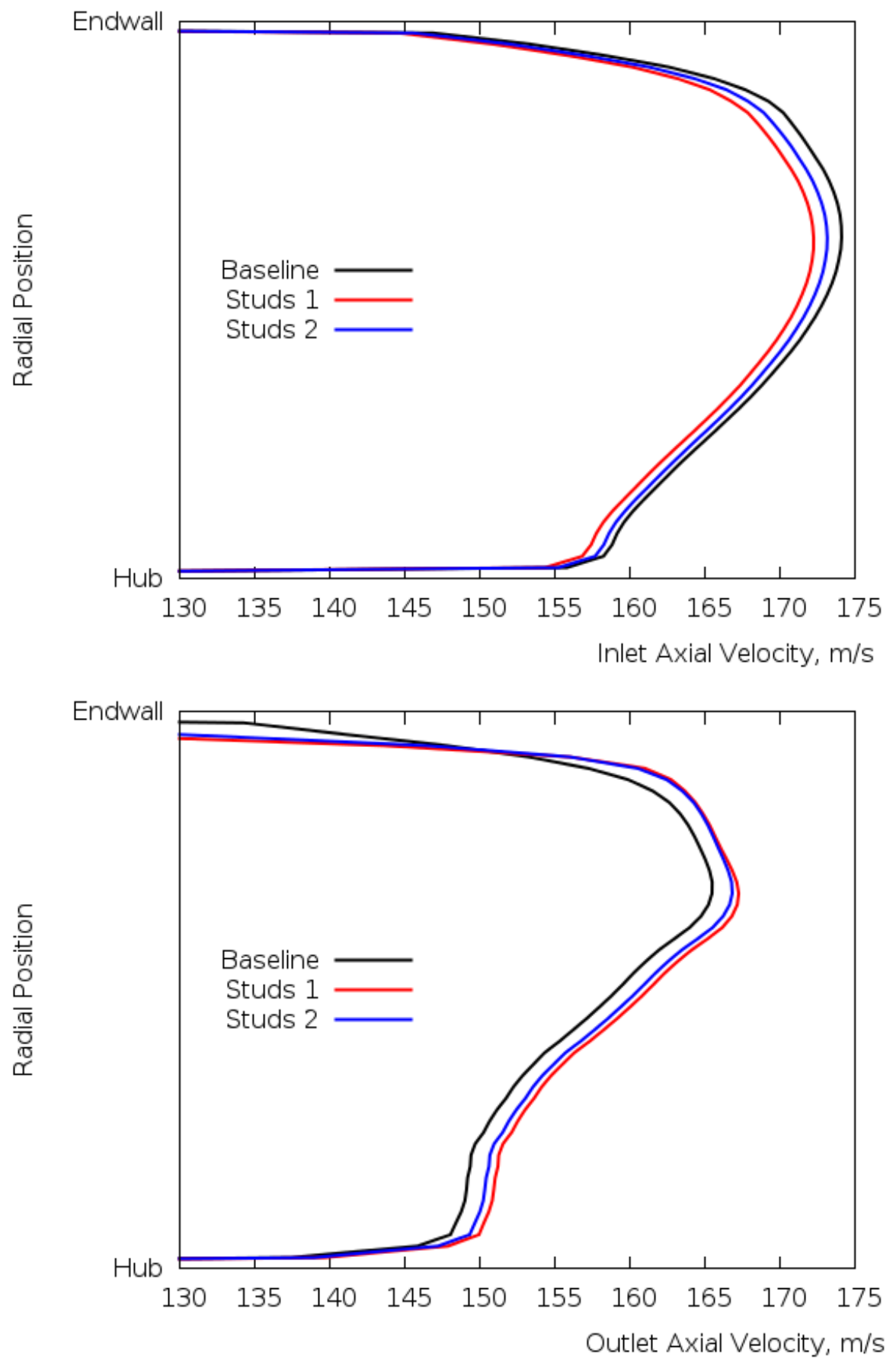

Figure 6.13 Radial distribution of axial velocities at $131 \mathrm{kPa}$ back pressure. 


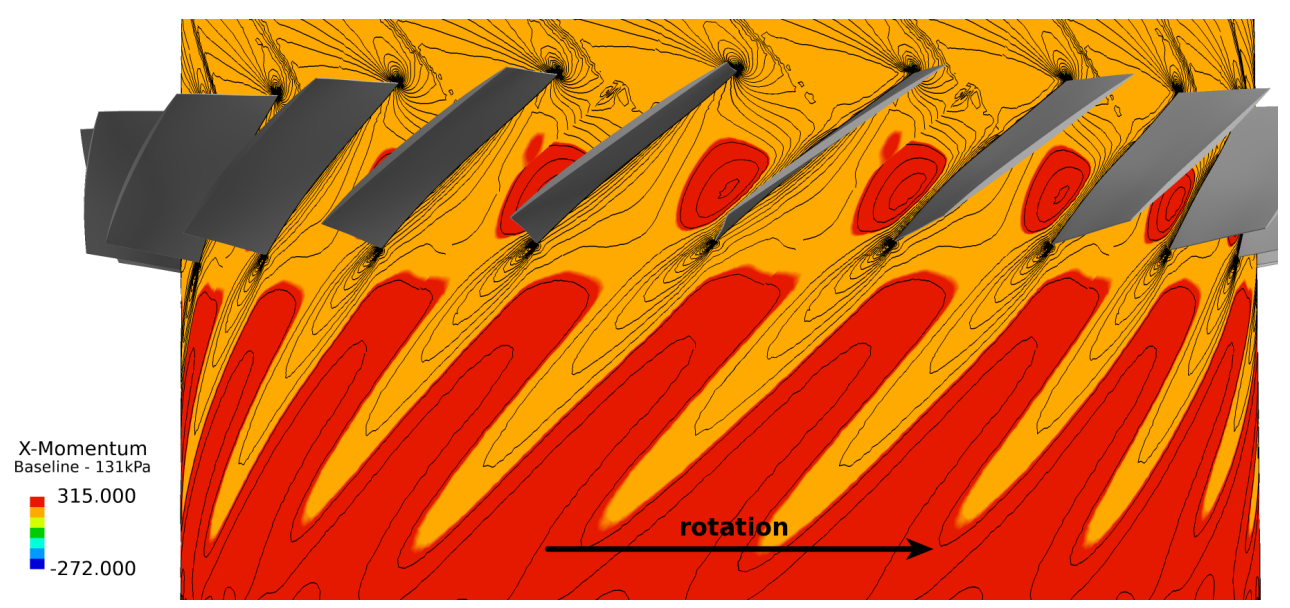

(a) Baseline

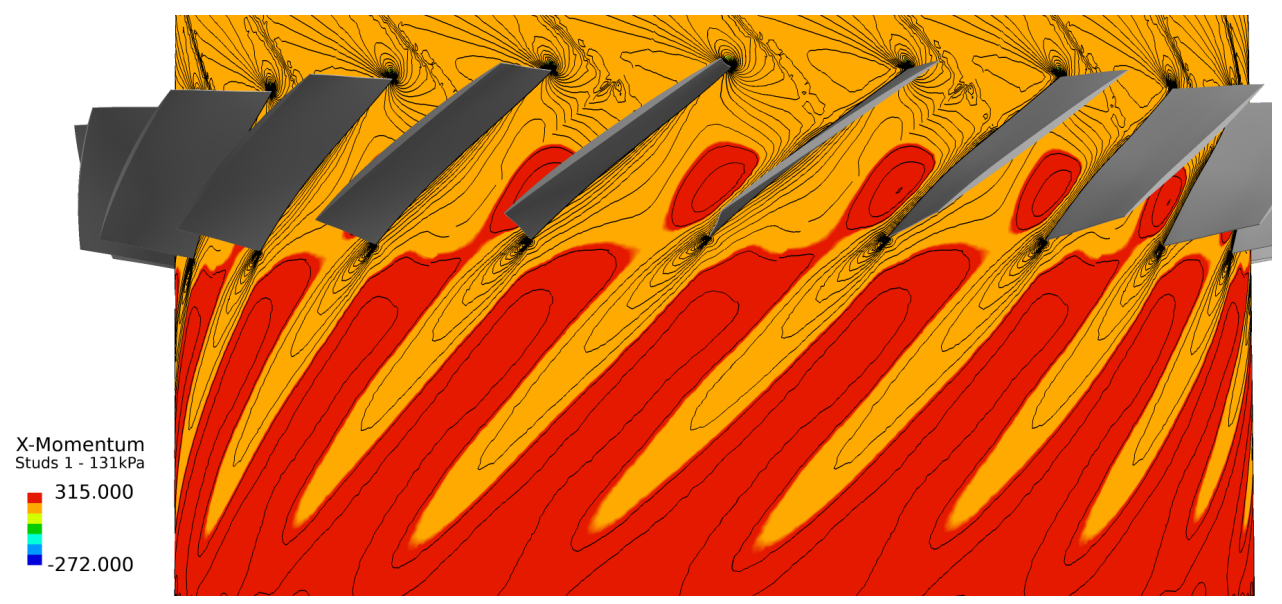

(b) Studs 1

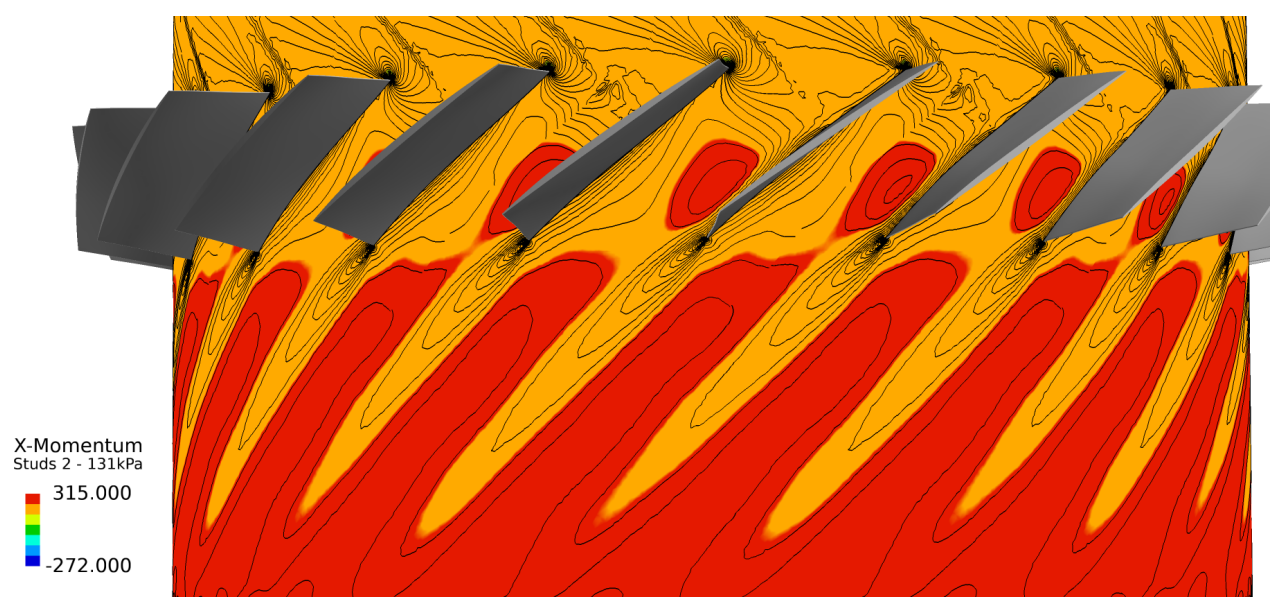

(c) Studs 2

Figure 6.14 Comparison of axial momentum at a 75\% fan blade span (78\% outlet plane span) radial cut at $131 \mathrm{kPa}$ back pressure. 
Radial Distribution of Axial Velocity - $131.7 \mathrm{kPa}$
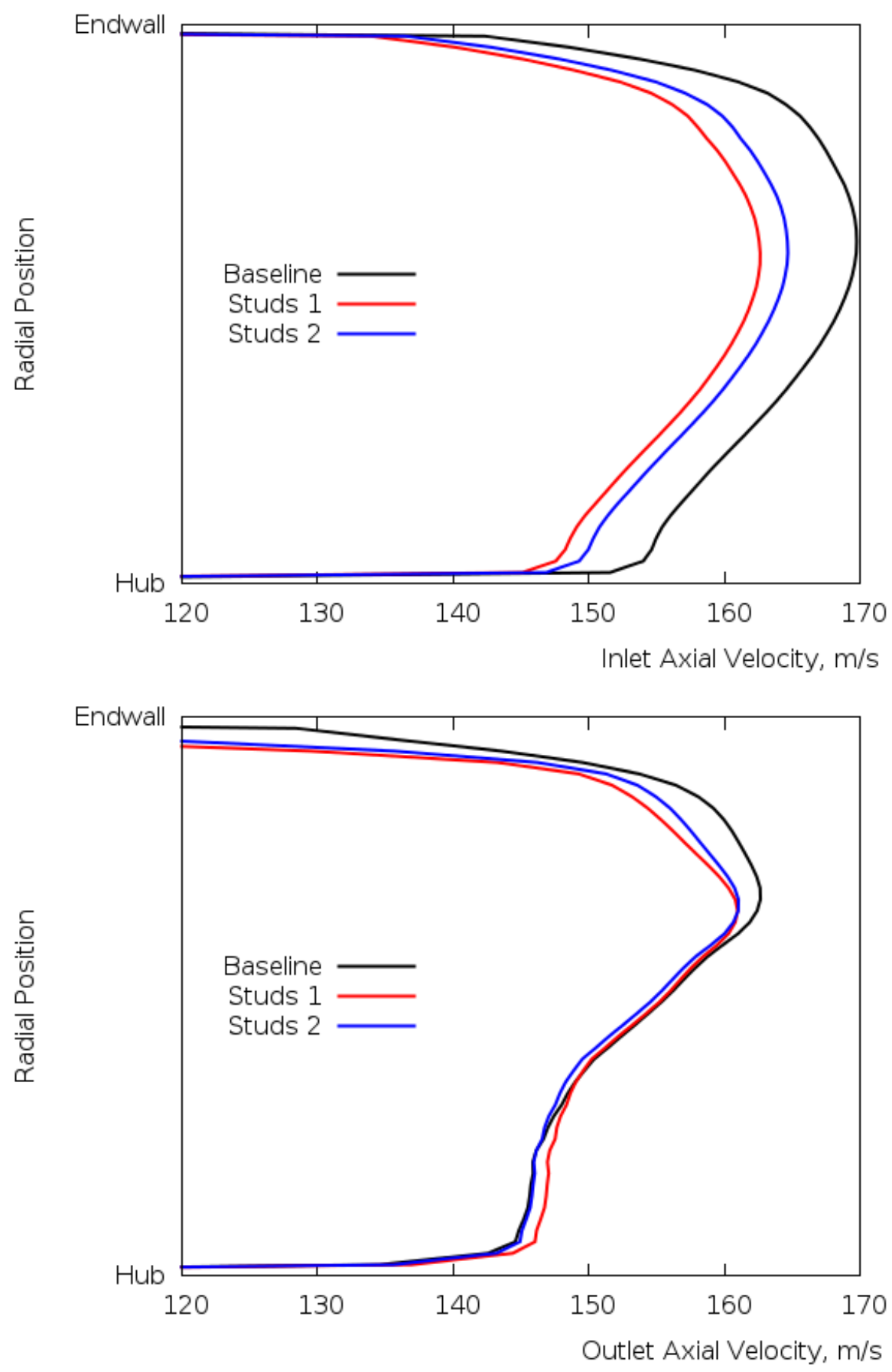

Figure 6.15 Radial distribution of axial velocities at baseline computed near stall conditions. 


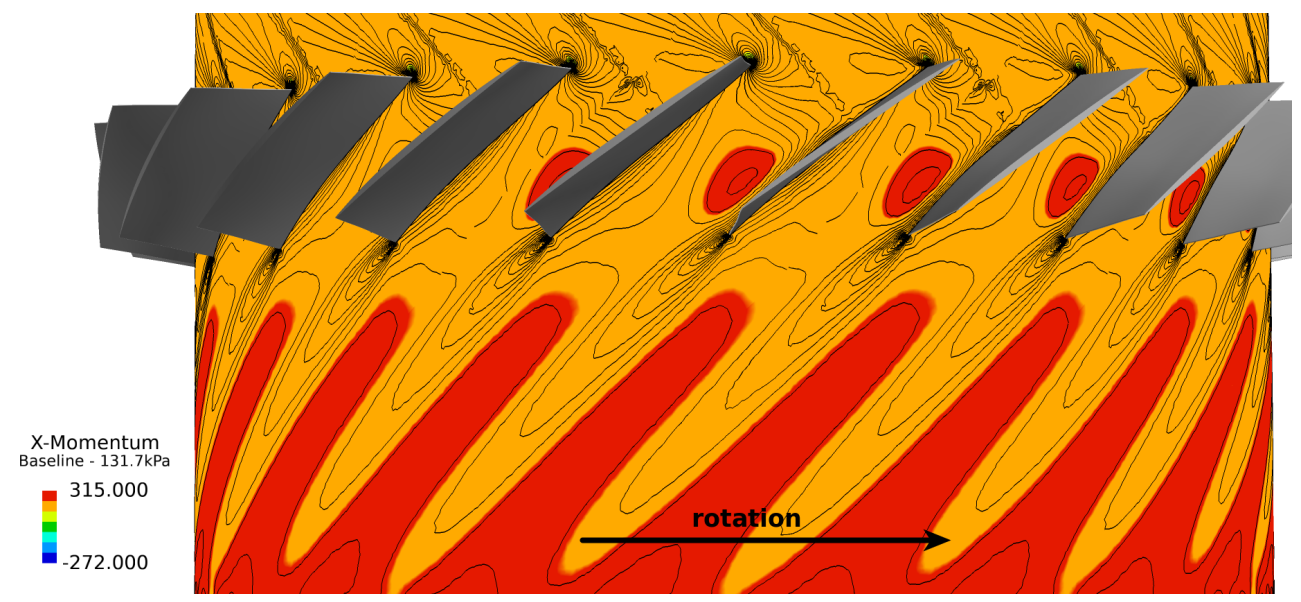

(a) Baseline

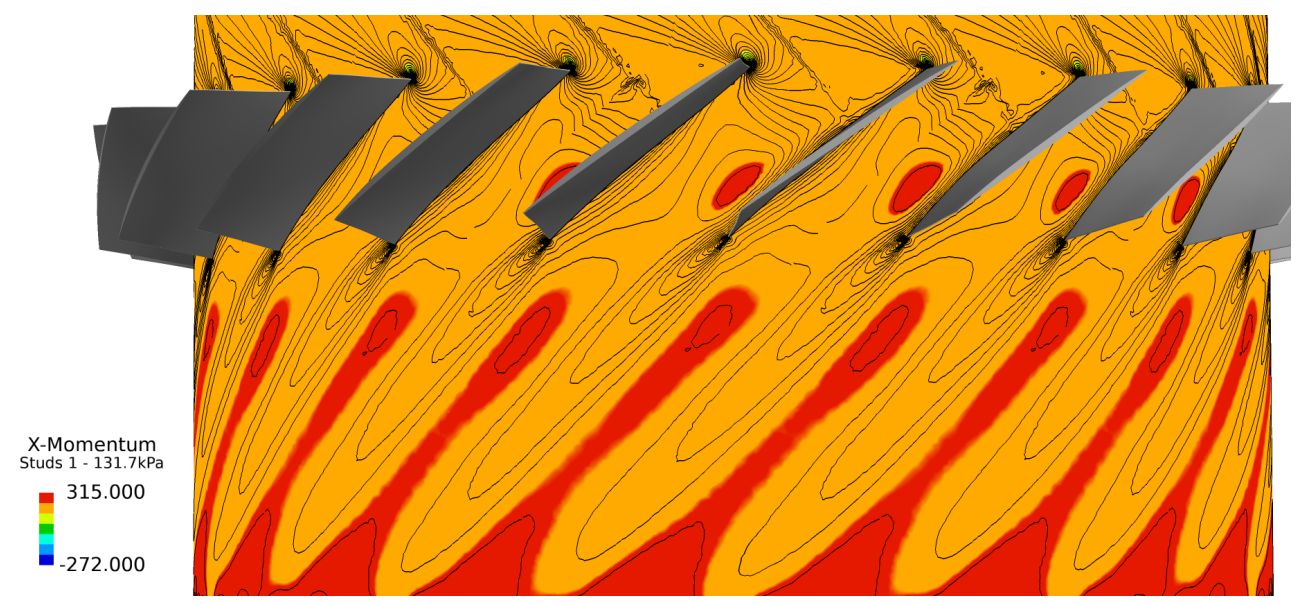

(b) Studs 1

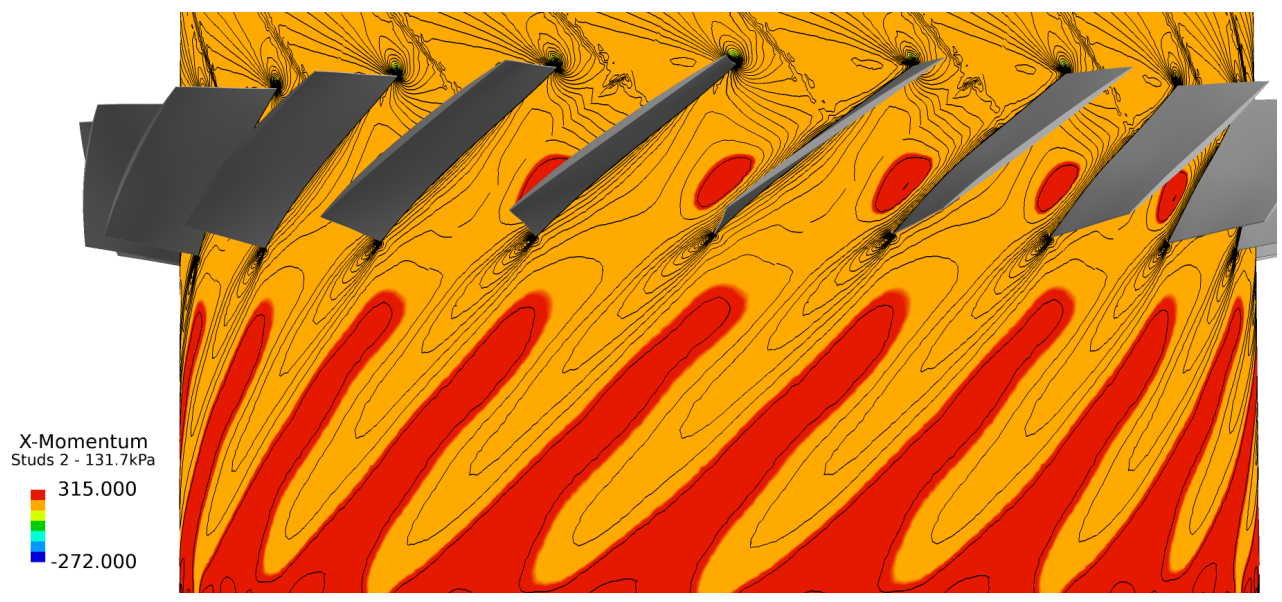

(c) Studs 2

Figure 6.16 Comparison of axial momentum at a 75\% fan blade span (78\% outlet plane span) radial cut at $131.7 \mathrm{kPa}$ back pressure. 


\subsubsection{Radial Distribution of Relative Flow Angles}

Averaged measurements were also taken of relative flow angles. Here, a relative flow angle is defined as the angle formed between a ray in the x-direction at a point (parallel to the axis of rotation) and the vector at that point that is equal to the relative velocity vector minus its radial velocity component. This definition is depicted in Figure 6.17. Figure 6.18 gives the radial distribution of the measurements for cases of $122.5 \mathrm{kPa}$ and $131 \mathrm{kPa}$ back pressures. These figures are a close up of the upper portion since this is where the greater differences show, but the profile of the entire span is provided in the insets. Only the measurements taken at the outlet plane are shown because the inlet plane measurements showed negligible changes. The results provided here show that the flow angles are affected by the presence of the casing treatment, as would be expected. The higher relative flow angles mean relatively smaller velocity components in the tangential direction of the flow near the casing. In other words, from observation of Figure 6.18 , the casing treatment causes a decrease of the flow velocity in the tangential direction near the casing and a slight acceleration away from it. The turning point of flow "faster than" to "slower than" the baseline case in the tangential direction happens approximately at $90 \%$ of the outlet plane span, and this effect seems to be only slightly greater for the Studs 1 configuration.

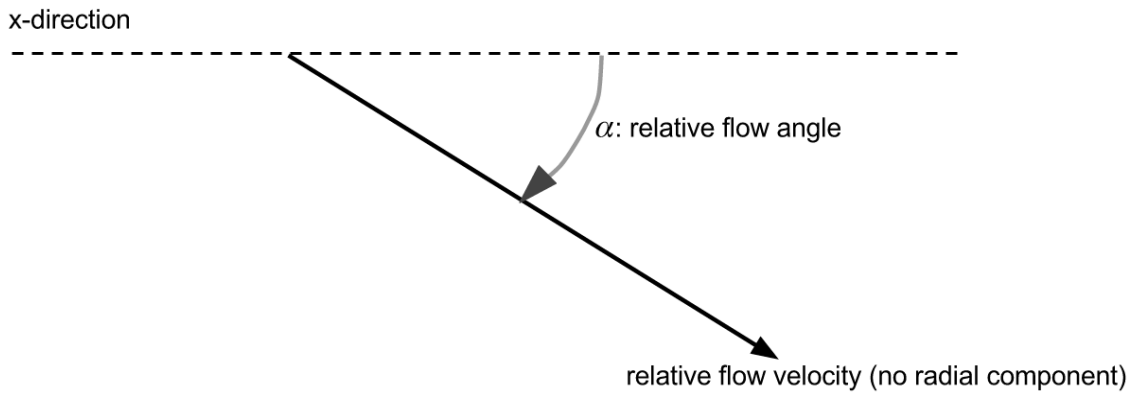

Figure 6.17 Definition of relative flow angle. 


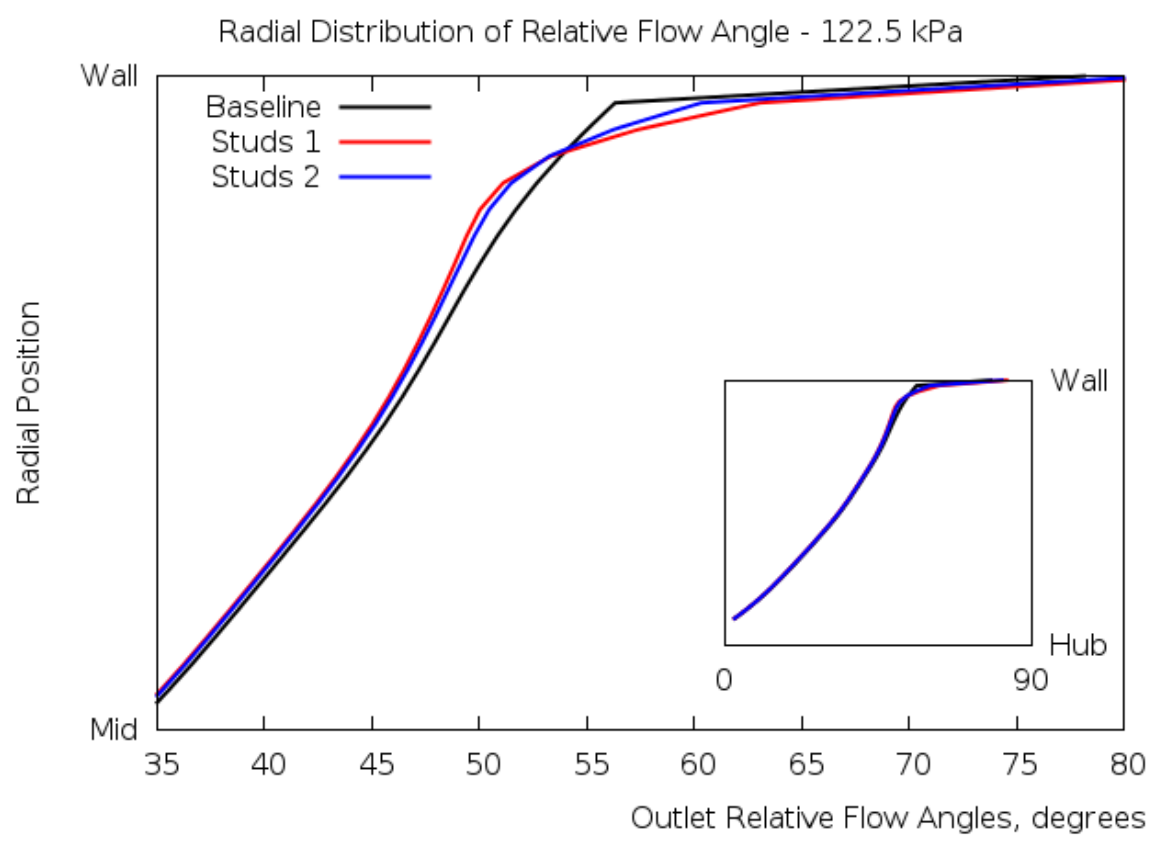

(a) Relative flow angles at computed operating point with highest efficiency.

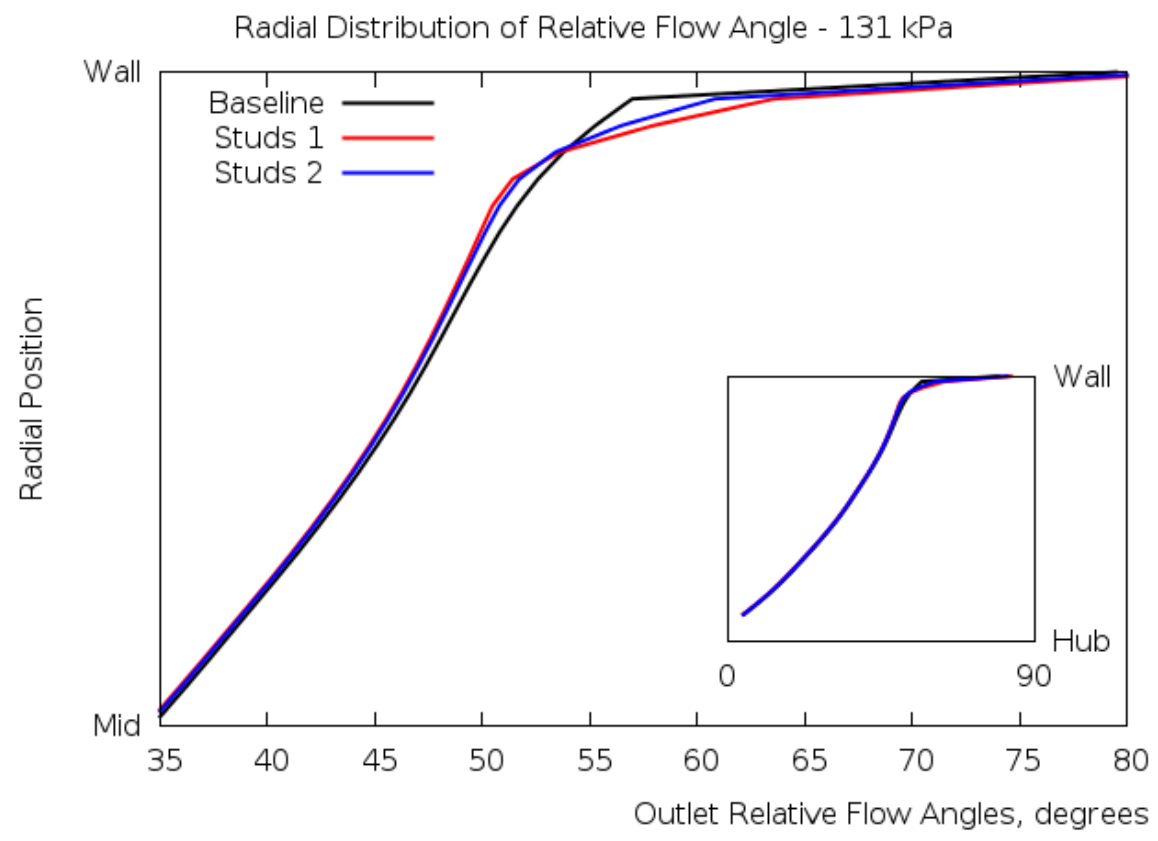

(b) Relative flow angles at $131 \mathrm{kPa}$ back pressure operating point.

Figure 6.18 Comparison of relative flow angles at outlet plane. 


\subsubsection{Flow-Field Features at 131.7 kPa Back Pressure}

A close up view of the flow features is provided by Figure 6.19. It provides radial cuts colored by relative Mach number and located at $97.8 \%$ blade span, which is approximately at half of the protrusion level of the studs. The back pressure condition is $131.7 \mathrm{kPa}$, which is the last stable operating point tested for the baseline case. Figure 6.19 is analogous to Figure 5.13 in section 5.2. It serves to visualize regions of low momentum in the fan blade passage. In this case, it also serves to visualize the effects of the studs on the flow-field near the casing. The use of contour lines is avoided here in order to improve the visibility of the streamlines.

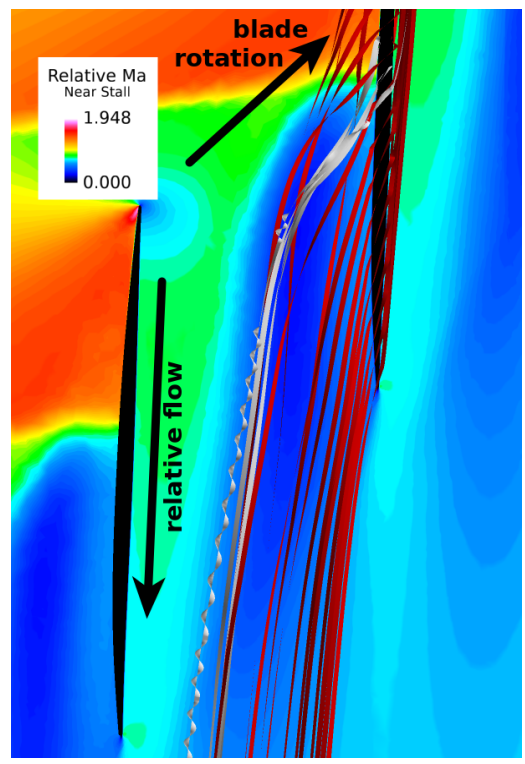

(a) Baseline

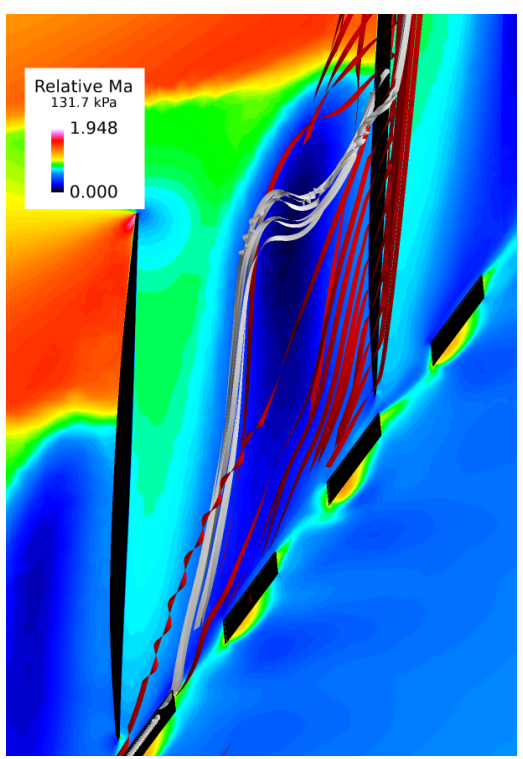

(b) Studs 1

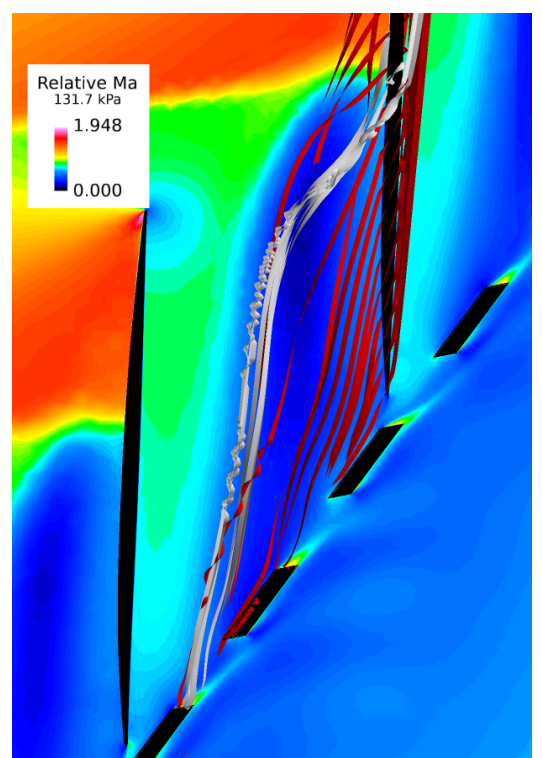

(c) Studs 2

Figure 6.19 Comparison of relative Mach number in passages between baseline and casing treatment simulations, where the cuts are located at mid height of the studs ( $97.8 \%$ span). 
The left-most picture in Figure 6.19 is for the baseline configuration with no casing treatment, the center picture is for the case with Studs 1, and the right-most picture is for the case with Studs 2. The red ribbons were seeded along the blade tip gap in order to track the path of the flow. The white ribbons represent streamlines seeded on various radial surfaces near the one shown, in spots where relative Mach number values were the lowest. The intention was to trace the origin of the fluid that passes through the regions of lowest momentum. Relative velocity vectors were used in the calculation of all streamlines.

From comparison of the radial cutting planes, it is observed that the region of low relative Mach number present in the baseline case is exacerbated in the presence of the protruding studs. Such regions have further expanded across the passage and display darker coloring (indicating lower relative Mach number) in comparison with the coloring of the baseline case. In addition, this effect on the relative Mach number seems to be stronger with the use of Studs 1 .

The following observations can be made from comparing the streamlines across all cases. First, in all cases the flow represented by the white ribbon streamlines seem to originate from a location in the gap just downstream of the location of the shock. Second, for all cases, the flow represented by the red ribbons seems to take a turn around the core of a vortex and seems to originate in a location upstream of the shock. If the streamlines originate downstream of the location of the shock, their path seems to be straight and downstream. Third, the streamlines follow a more erratic path in the presence of the protruding studs, changing directions and taking more turns, most noticeably for the white ribbon streamlines. Finally, the streamlines seem to be driven below the radial surface when and away from the casing in the presence of the casing treatment, where as, in the baseline case, the streamlines continue their path near the casing. This suggests 
that some of the lower momentum flow is being driven into the higher energy flow regions, away from the casing. The range extension suggested by the simulations could be partially credited to this observation.

Isosurfaces of $v$, provided in Figure 6.20, were created at a value of $6000^{m^{2}} / s$ in order to proceed with an analysis similar to that presented in subsection 5.2.3. A profile view of the isosurfaces created for the three cases is presented together in order to compare the effects of the casing treatment. The flow moves from right to left. The orientation of the blades was chosen in order to provide a better view of the isosurfaces.

By careful observation of the fan blade passage region near the casing, the main difference noticed is the increase in extension of the low momentum fluid, as it was mentioned in the analysis of Figure 6.19. Also, it is observed that there is a low momentum region near the casing that extends downstream of the studs (it extends further when using Studs 1). These observations suggest that the presence of the studs produces a large blockage near the casing, which extends from the fan blade passage to the region between the fan blades and the stator vanes. This blockage has the effect of redistributing the amount of fluid moving at different locations of the duct as if the duct geometry itself was being changed.

Changing focus to the stator region and downstream, it is observed that the low momentum region that existed near the hub in the baseline case at this back pressure has mostly diminished when using casing treatment. Now a large low momentum region has appeared closer to the casing for both casing treatment cases. This effect can be correlated to the findings reported on axial velocities and relative flow angles in subsections 6.2.1 and 6.2.2, respectively. It is believed that 
the decreased speed of the fluid near the casing is largely responsible for this effect on the stator region.

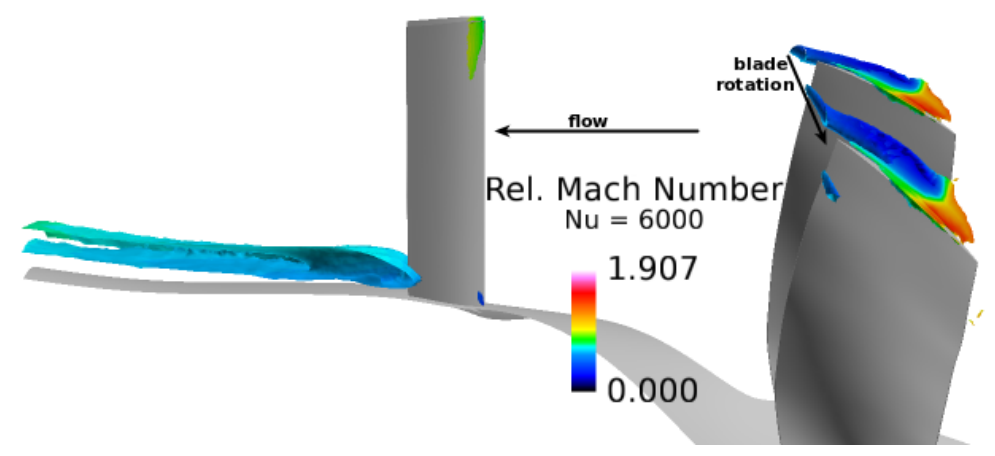

(a) Baseline

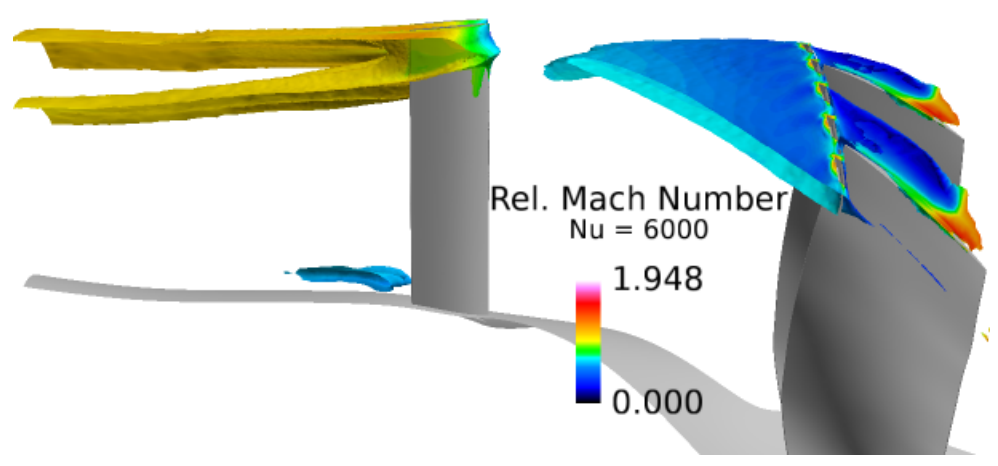

(b) Studs 1

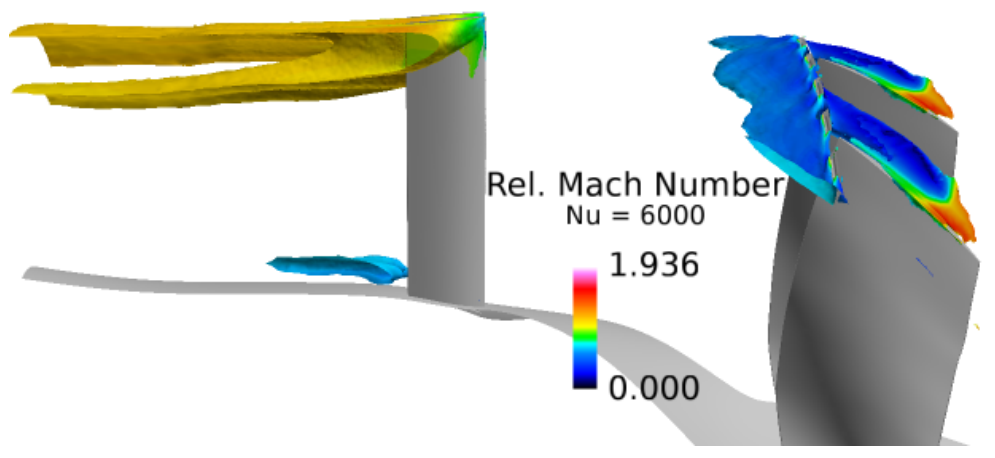

(c) Studs 2

Figure 6.20 Isosurface of $v$ side view comparison. 
Next, Figure 6.21 is presented depicting ribbon shaped streamlines for both casing treatment cases, seeded in a similar way to those in Figure 5.19a. It is observed that the pattern of flow within the low momentum region in the flow passage is very random in both cases. There appears to be a greater tendency toward recirculation due to the adverse pressure enhanced by the presence of the studs. In this case, the flow tending toward recirculation seems to originate not only from the tip gap region but also from upstream of the blade leading edge. In addition, as in the baseline case, there is flow originating at the tip gap that crosses the gap of the adjacent blade. This time, however, the streamlines depicting flow crossing more than one tip gap did not need to be seeded only at specific locations of the gap as in the baseline case. In other words, the computations show a greater tendency for flow crossing more than one passage. 


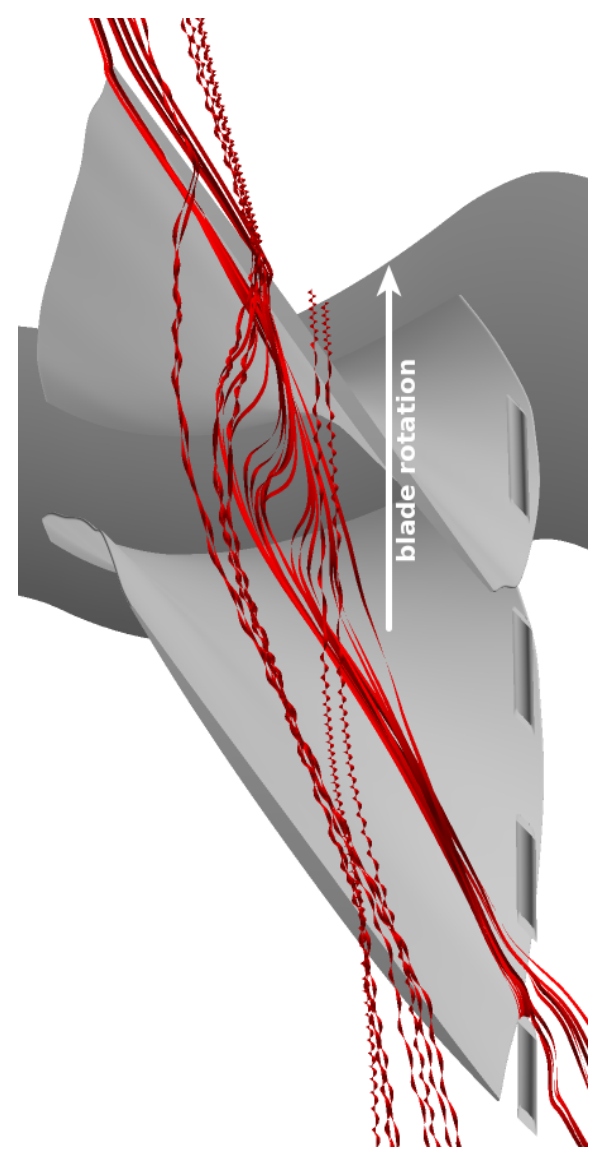

(a) Studs 1

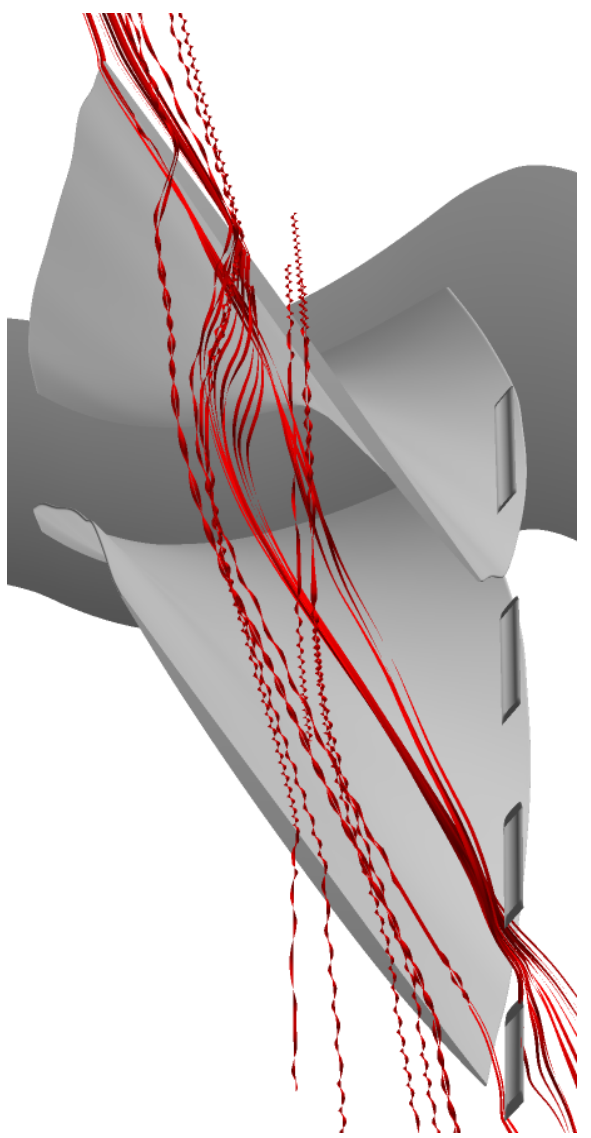

(b) Studs 2

Figure 6.21 Patterns of tip gap flow using casing treatment at $131.7 \mathrm{kPa}$ back pressure.

Finally, Figure 6.22 shows streamlines seeded on the $v$ isosurfaces presented in Figures $6.20 \mathrm{~b}$ and $6.20 \mathrm{c}$ in the stator region. It is observed that a strong recirculation on the suction side of the blades near the casing has now appeared. This new flow feature was not observed in the baseline case, but now appears for both types of studs. It is attributed to the decrease in axial velocity near the casing, which was reported in sections 6.2.1 and 6.2.2, in combination with the adverse pressure gradient. 


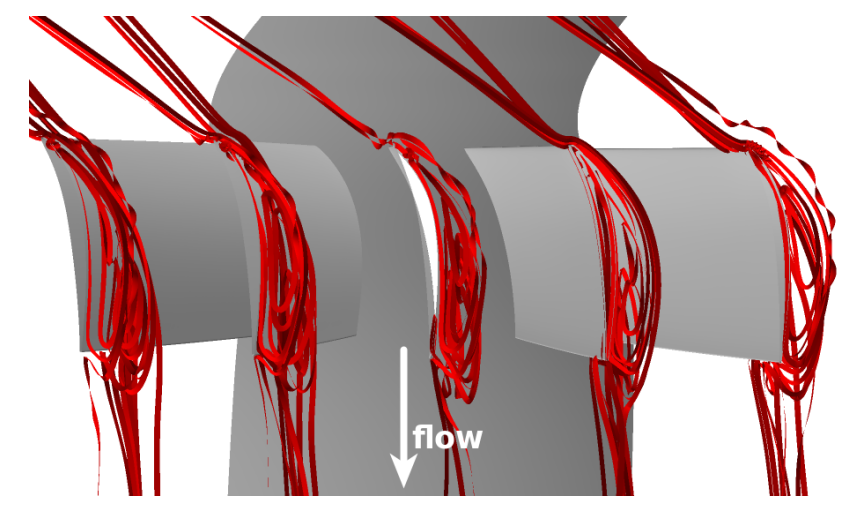

(a) Studs 1

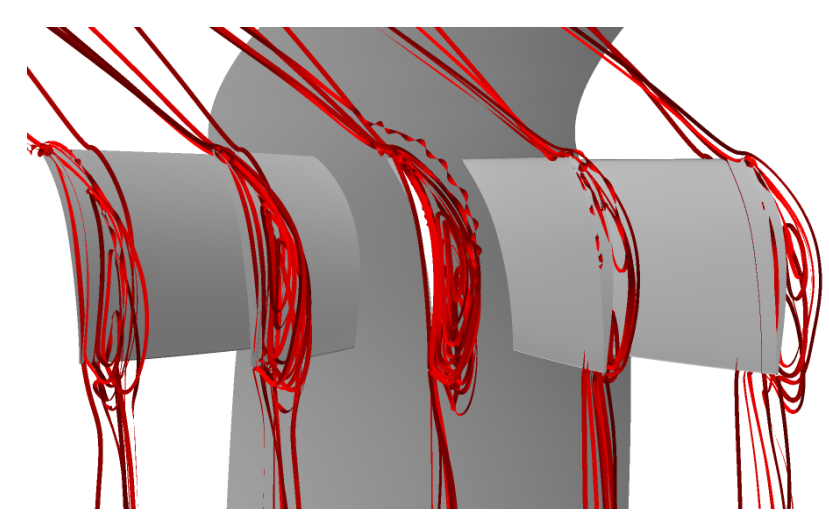

(b) Studs 2

Figure 6.22 Recirculation on suction side of stator blades near casing for $131.7 \mathrm{kPa}$ back pressure.

\subsubsection{Flow-Field Features at New Near-Stall Conditions With Casing Treatment}

This subsection provides an analysis similar to that of subsection 6.2.3, using isosurfaces of $v$ and streamlines. The operating conditions are $132.2 \mathrm{kPa}$ back pressure for the Studs 1 case and $132.4 \mathrm{kPa}$ back pressure for the Studs 2 case. These conditions are the last stable point tested and presented in the performance curves. They represent the new near-stall conditions for each stud configuration. It should be noted that although the back pressures differ by $200 \mathrm{~Pa}$, the mass 
flow rates are very similar for each case at approximately $84.8 \mathrm{lbm} / \mathrm{s}$ which is very convenient for comparison.

Figure 6.23 displays isosurfaces of $v$ created for both stud cases in similar fashion as that created for the baseline case in Figure 5.18a. It is observed that now the low momentum region near the casing, as encased by the isosurface, extends from the blade flow passage, through the stator vanes, and all the way to the outlet plane. In addition, low momentum regions near the hub and downstream of the stator vanes have reappeared. The general observation from Figure 6.23 is then that the flow has remained stable until reaching this operating condition where the duct is constricted by very large regions of low momentum fluid.

Figure 6.24 displays the streamlines seeded on the $v$ isosurfaces in the fan blade passage region for both stud cases. In addition to some flow spilling into the adjacent passage, strong recirculation is observed. The fluid spilling and recirculating originates upstream of the blades and also at the tip gap region in locations upstream of the shock. Similarly, in Figure 6.25 for the stators, the recirculation observed at $131.7 \mathrm{kPa}$ back pressure operating condition has become stronger in the new near-stall operating condition. A final observation is that the flow patterns depicted by the streamlines at the fan blade passage and at the stator region for both studs configurations are very similar. 


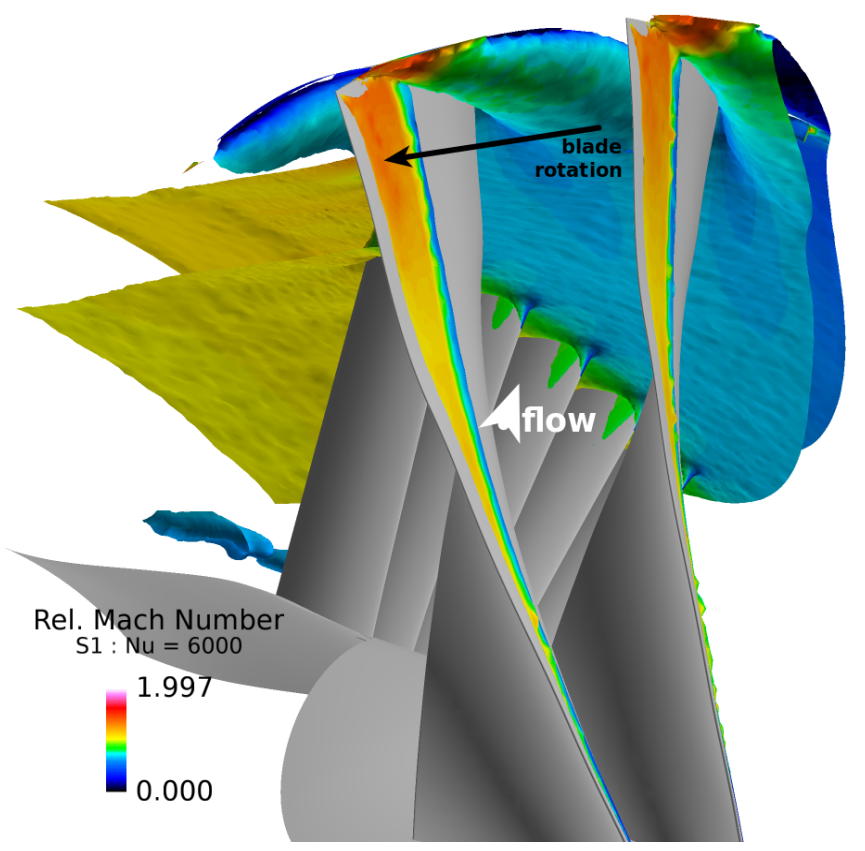

(a) Studs 1

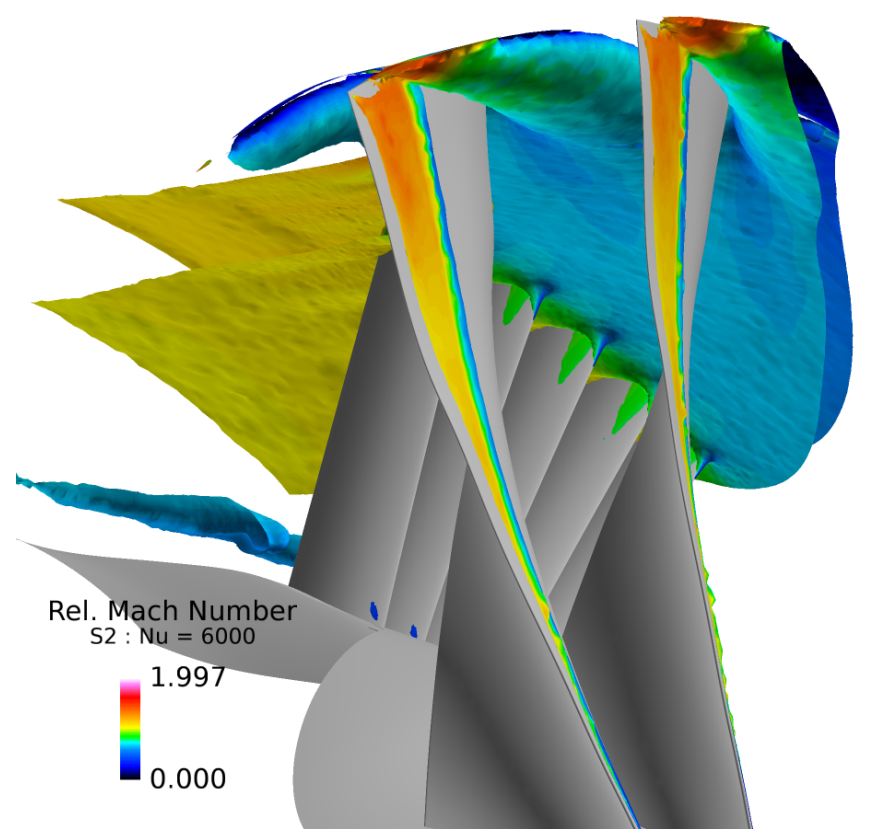

(b) Studs 2

Figure 6.23 Forward-looking-aft view of isosurfaces of $v$ at near stall conditions using Studs 1 and Studs 2. 


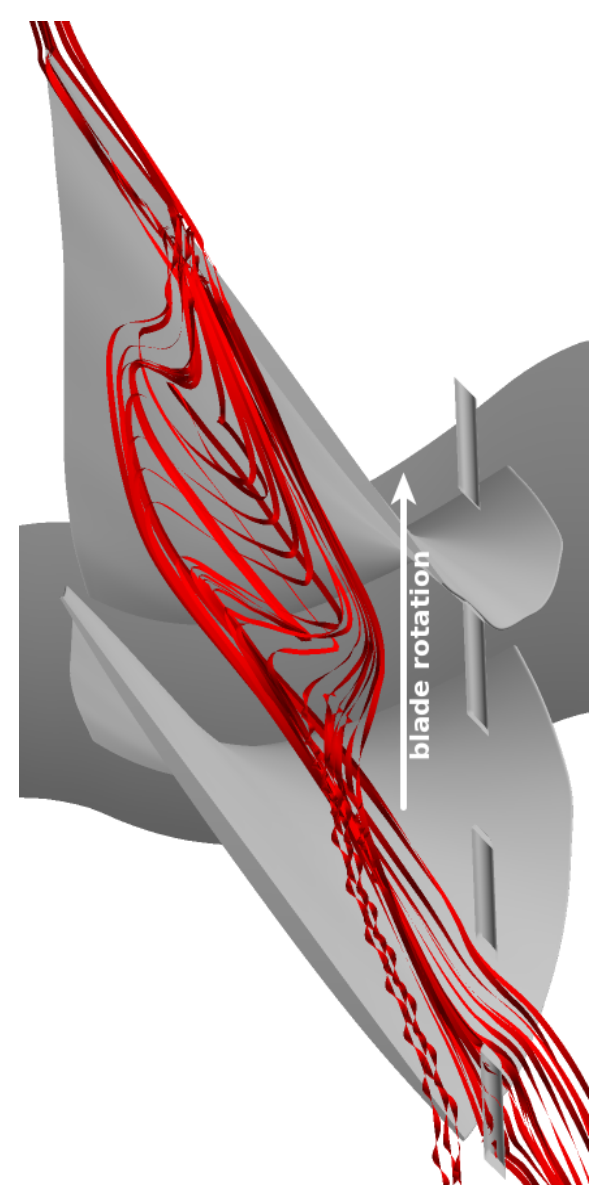

(a) Studs 1

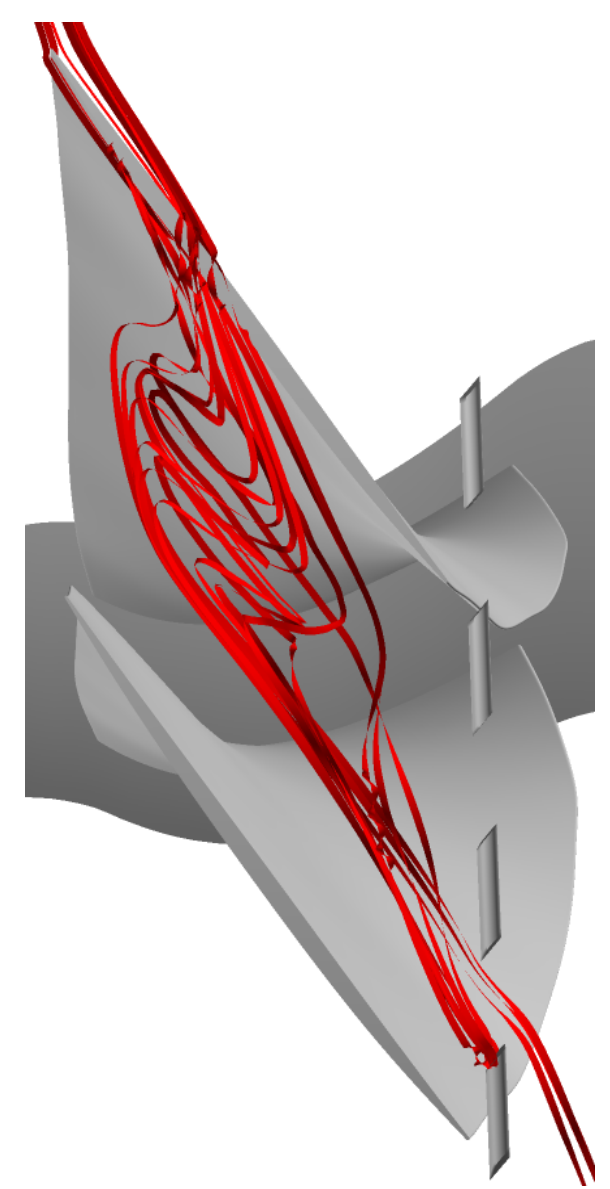

(b) Studs 2

Figure 6.24 Patterns of tip gap flow using treatment at near stall conditions. 


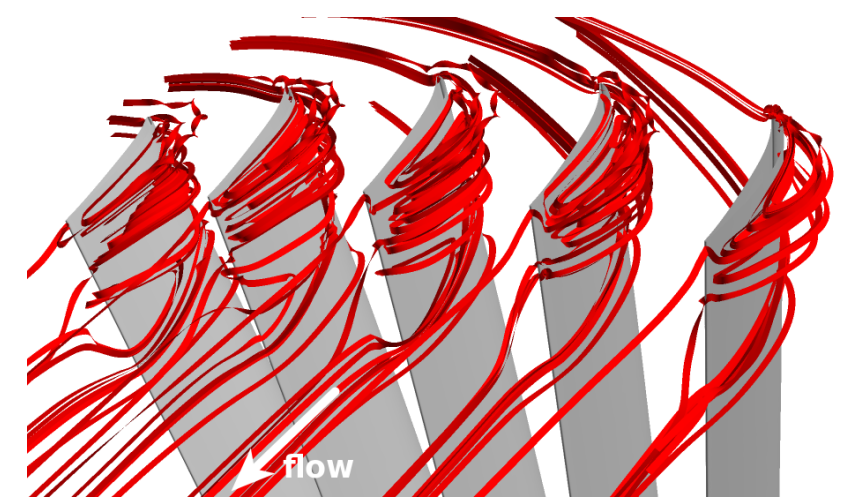

(a) Studs 1

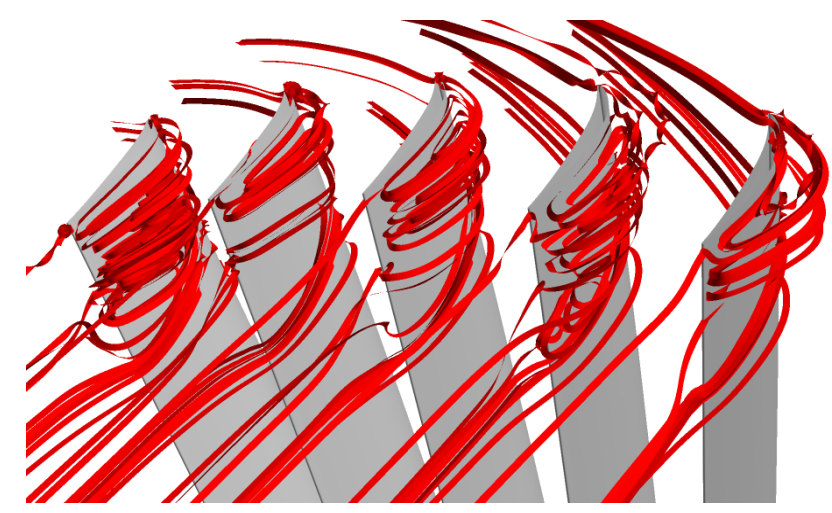

(b) Studs 2

Figure 6.25 Recirculation on suction side of stator blades near casing for near stall conditions.

\subsubsection{Studs 1 vs. Studs 2}

Next is Figure 6.26, which is a radial isosurface colored by total temperature and at half way the protrusion of the studs ( $97.8 \%$ blade span). Solutions for this figure were calculated at $131.7 \mathrm{kPa}$ back pressure. The view on the left image is of the Studs 1 casing treatment, and Studs 2 is on the right. The different color patterns between the cases reveals that the total temperature increase is higher for Studs 1. This observation was made in subsection 6.1.1, where the total temperature rise curves were presented. This effect can be attributed to the fact that the flow interaction with Studs 
1 is more harsh than with Studs 2, as depicted by Figure 6.27. In this figure, flow velocity vectors are displayed as one fan blade passes by both studs. It is observed on the left image that as the flow interacts with a stud from the Studs 1 configuration, flow recirculation occurs on the downstream face of the structure. In the case of flow interacting with a stud of the Studs 2 configuration, shown in the image on the right, the interaction appears to be more favorable because the flow is less likely to separate from the surface of the structure. Figure 6.28 shows flow patterns around the studs and serves to confirm the these observations.

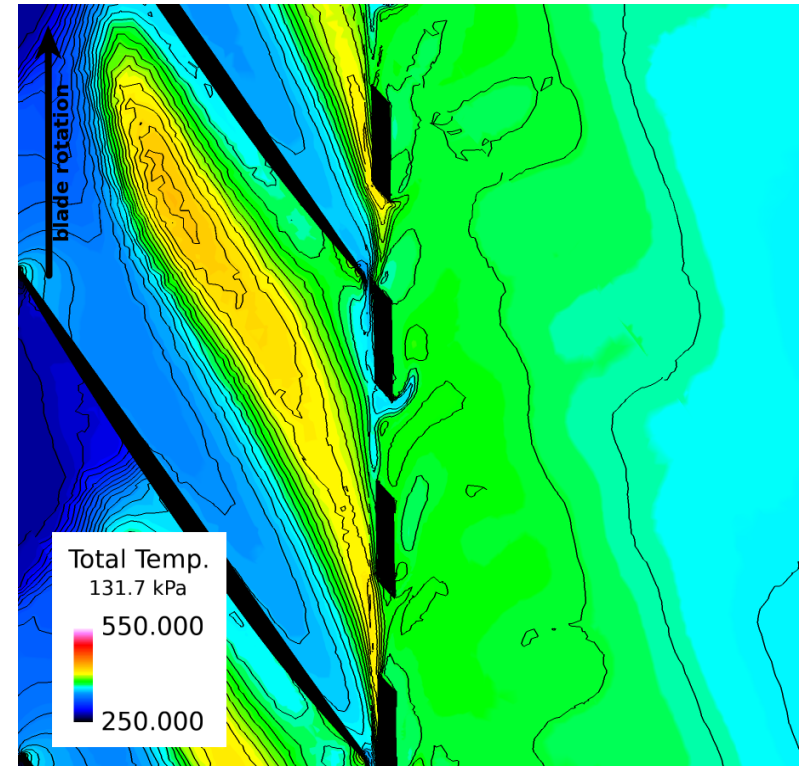

(a) Studs 1

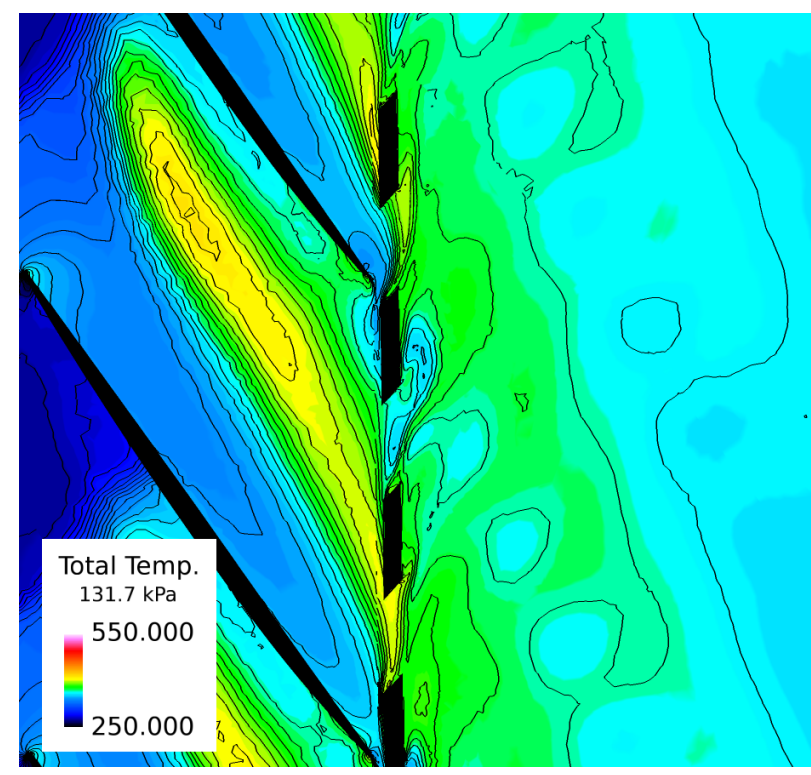

(b) Studs 2

Figure 6.26 Comparison of total temperature coloration across Studs 1 and Studs 2, where the radial cuts are located at mid height of the studs ( $97.8 \%$ span). 


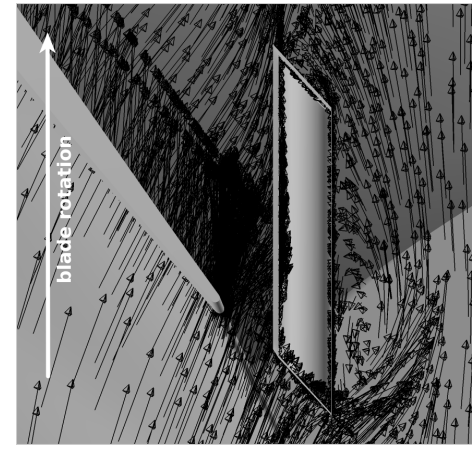

(a) Studs 1

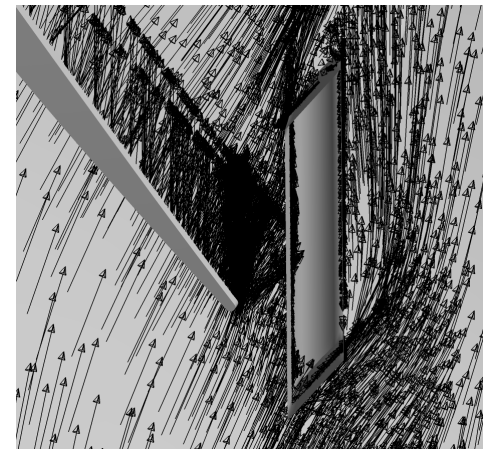

(b) Studs 2

Figure 6.27 Comparison of velocity vectors around Studs 1 and Studs 2, where the radial cuts at mid height of the studs (97.8\% span).

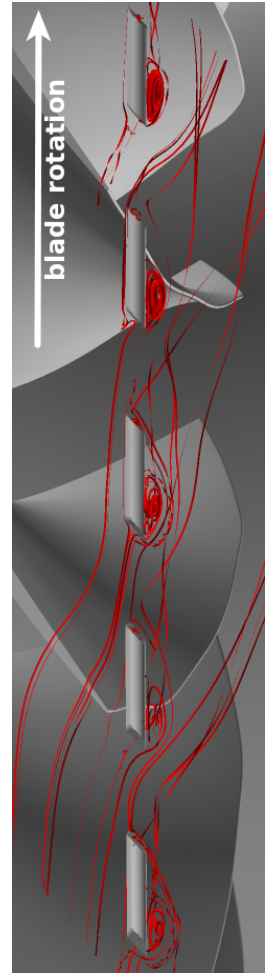

(a) Studs 1

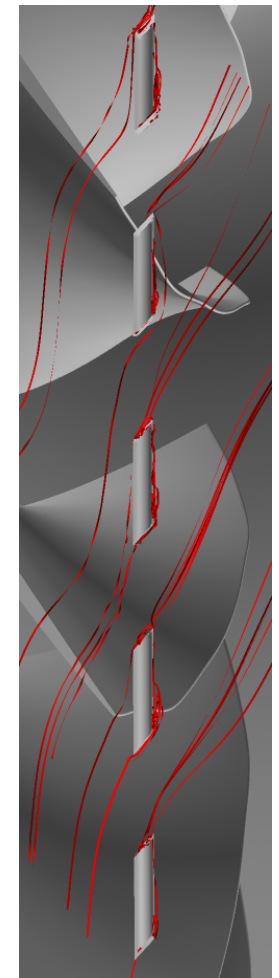

(b) Studs 2

Figure 6.28 Flow patterns around Studs. 


\section{CHAPTER 7}

\section{CONCLUDING REMARKS}

\subsection{Conclusions}

The following is a summary of the research performed in the study of protruding studs as a form of casing treatment:

- The performance measures obtained from experiments on a transonic turbofan have been reproduced by numerical simulations with a significant degree of success. The performance curves of the fan alone were approximated very well for the $100 \%$ of design speed, and adequately for the $87.5 \%$ of design speed. In the case of the stage performance (rotor and stator) the results of the simulation agree well with the reported performance with one consideration. The reproduction of the efficiency curves seems adequate when the computed stage temperature ratios are used in the calculations instead of the fan temperature ratios, as was done in the experiments. The computed stage efficiency curves reflect the trend but do not reproduce exactly the reported efficiency curves if the computed fan temperature ratios are used.

- A suitable computational mesh was created for carrying out the turbofan simulations that would also lend itself to the introduction of the casing treatment while introducing minimal changes in the mesh. It was found that performing this task was very labor intensive, and 
it required a certain degree of skill and experience. Many computational meshes had been created before arriving at one that allowed acceptable reproduction of experimental results. It was found that the number of viscous layers has a significant impact on the solutions, along with the point distribution on the surfaces.

- From visualization of the computed flow-field, locations of low momentum flow from boundary layer buildup have been localized by coloring of isosurfaces with relative Mach number and relative momentum. Tip gap flow was tracked through streamlines to identify its path and visualize its interaction with the flow in low momentum regions. It has been observed that flow crossing the tip gap in locations of shock interaction with the blade suction surface also pass through the spots of lowest relative Mach number. It has also been observed that tip gap flow passing through locations upstream of the location of the shock have a tendency to make turns forming a vortex within the cells. In addition, streamlines seeded along the tip gap, very close to the casing, show flow also crossing the tip gap of adjacent fan blades.

- A form of casing treatment was tested via simulations, which suggest that it has a potential for increasing the range of operation of the compression system. The casing treatment consists of protruding studs in the shape of tangentially elongated "U's." Two configurations were tested that differed in the alignment of the sides: in one the sides were aligned with the tip of the fan blade, while in the other, the sides were aligned opposite (see Chapter 5). The computations suggest that the protruding studs tested can increase the computed range of the turbofan between 5\% and 5.5\%. The pressure rise and efficiency costs from using this casing treatment are not high, and the second type of studs seems to be slightly more efficient. 
- Measurements and visualizations of the flow-field suggest the mechanisms for stability enhancement. First, it has been observed that the studs enforce a blockage in the location where a stall cell appeared when using no casing treatment. This forces fluid to flow under the studs and to accelerate. It has been observed through streamlines that tip gap flow, that would become part of stall cells, gets diverted away from the casing by the studs (Figure 6.19). It is thought that the tip gap flow ends up mixing with higher energy flow passing under the studs(Figure 6.15). Second, through visualization of kinematic viscosity isosurfaces, it was also observed that the presence of the studs creates regions of low momentum flow near the casing downstream of the studs, and the extension of these regions increases further downstream as the outlet back pressure condition is increased. At $131.7 \mathrm{kPa}$ back pressure operating condition, the low momentum regions near the casing extends nearly to the location of the stator vanes. This causes a low momentum region downstream of the stators near the hub (Figure 5.20b) to disappear or to decrease in extent (Figure 6.20). At the new near-stall operating conditions reached using the original studs $(132.2 \mathrm{kPa}$ and $132.4 \mathrm{kPa}$ for Studs 1 and Studs 2, respectively), it is observed through kinematic viscosity isosurfaces that the low momentum regions near the casing extend from the rotor blade passage to the outlet plane, and that the low momentum region downstream of the stators near the hub appear with a greater extension (Figure 6.23). From this it is thought that the low momentum region downstream of the stator near the hub may have an influence in the stalling of the fan stage when using protruding studs. 
- Comparison of performance curves using casing treatment, and visualization of total temperature, velocity vectors at mid span and streamlines reveal that the shape of the studs with sides aligned opposite to the stagger angle of the rotor blades are more efficient the studs with sides aligned with rotor blade stagger angle.

- Variations of the original studs by protrusion level, extension in tangential direction, and a different shape were tested. The goal was to assess the impact on performance from these variations. From comparison of the performance curves, it was observed that varying degrees of increased stall margin can be obtained by changing the protrusion level of the studs.

\subsection{Recommendations}

From the foregoing summary, the following recommendations are made for future work:

- Perform a mesh convergence study.

- Perform perform simulations on a half or full wheel of the turbofan.

- Experiment with different numbers of viscous layers in the mesh by simulating the operation at various points of the performance curves.

- For the purpose of comparison at similar mass flow operating conditions, run simulations at slightly different back pressures in order to find operating conditions of matching mass flow.

- Further study the effects of protrusion levels.

- Study the impact of the location of the studs relative to the trailing edge of the rotor blades.

- Further analyze the mechanisms of stall at rotor and stator region when studs are in use. 
- Study structural effects of the interaction between rotor blades and studs.

- Use the findings from this study to create an optimal design and location of the protruding studs.

- Simulate protruding studs in other compression systems.

- An experimental study of this type of casing treatment on a compression system would be desired. 


\section{REFERENCES}

[1] White, F. M., Fluid Mechanics, Fifth Edition, McGraw-Hill, 2003.

[2] Hughes, C. E., "Aerodynamic Performance of Scale-Model Turbofan Outlet Guide Vanes Designed for Low Noise," 40th Aerospace Sciences Meeting and Exhibit, AIAA, Reno, NV, Jan. 2002.

[3] Lin, W., Computational Investigation of the Effects of Casing Treatments on the Performance of a Turbofan, Master's thesis, University of Tennessee, Chattanooga, May 2013.

[4] Wikipedia, "Wikipedia, The Free Encyclopedia," 2017, URL: http://www.wikipedia.org.

[5] Greitzer, E. M., "Surge and Rotating Stall in Axial Flow Compressors - Part I: Theoretical Compression System Model," Journal of Engineering for Power, Vol. 98, No. 2, April 1976, pp. 190-198.

[6] E. M Greitzer, J. P. Nikkanen, D. E. Haddad, R. S. Mazzawy, and H. D. Joslyn, “A Fundamental Criterion for the Application of Rotor Casing Treatment," Journal of Fluids Engineering, Vol. 101, No. 2, June 1979, pp. 237-243.

[7] E. M. Greitzer, "The Stability of Pumping Systems," Journal of Fluids Engineering, Vol. 103, No. 2, June 1981, pp. 193-242.

[8] E. M. Greitzer and F. K. Moore, "A Theory of Post-Stall Transients in Axial Compression Systems: Part II - Application,” Journal of Engineering for Gas Turbines and Power, Vol. 108, No. 2, April 1986, pp. 231-239.

[9] F. K. Moore, and E. M. Greitzer, "A Theory of Post-Stall Transients in Axial Compression Systems: Part I - Development of Equations," Journal of Engineering for Gas Turbines and Power, Vol. 108, No. 1, Jan. 1986, pp. 68-76.

[10] Moore, R. D., "Rotor Tip Clearance Effects on Overall and Blade-Element Performance of Axial-Flow Transonic Fan Stage,” Nasa-tp-2049, NASA Lewis Research Center, Cleveland, OH, Sept. 1982.

[11] C. Hah, J. Bergner, and H. P. Schiffer, "Tip Clearance Vortex Oscillation, Vortex Shedding and Rotating Instabilities in an Axial Transonic Compressor Rotor," ASME Turbo Expo 2008, Power for Land, Sea and Air, ASME, Berlin, Germany, 2008. 
[12] Biollo, R. and Benini, E., "Recent Advances in Transonic Axial Compressor Aerodynamics," Jan. 2013, URL: http://dx.doi.org/10.1016/j.paerosci.2012.05.002.

[13] Niazi, S., Numerical Simulation of Rotating Stall and Surge Alleviation In Axial Compressors, Ph.D. thesis, Georgia Institute of Technology, GA, 2000.

[14] S. Schlechtriem and M Lotzerich, "Breakdown of Tip Leakage Vortices in Compressors at Flow Conditions Close to Stall," ASME International Gas Turbine and Aeroengine Congress and Exhibition, ASME, Orlando, Florida, 1997.

[15] C. Hah, D. C. Rabe, and A. R. Wadia, "Role of Tip-Leakage Vortices and Passage Shock in Stall Inception in a Swept Transonic Compressor Rotor," ASME Turbo Expo 2004, Power for Land, Sea and Air, ASME, Vienna, Austria, 2004.

[16] S. Niazi, A. Stein, and L. N. Sankar, "Numerical Studies of Stall and Surge Alleviation in a High-Speed Transonic Fan Rotor," 38th Aerospace Sciences Meeting and Exhibit, AIAA, Reno, NV, 2000.

[17] Hathaway, M. D., "Self-Recirculating Casing Treatment Concept for Enhanced Compressor Performance," ASME Turbo Expo 2002: Power for Land, Sea, and Air, ASME, Amsterdam, The Netherlands, June 2002.

[18] A. Stein, S. Niazi, J. V. R. Prasad, and L. N. Sankar, "Numerical Simulations of compressor Flow Control," 18th Applied Aerodynamics Conference and Exhibit, AIAA, Denver, CO, 2000 .

[19] Skoch, G. J., "Experimental Investigation of Centrifugal Compressor Stabilization Techniques," Journal of Turbomachinery, Vol. 125, No. 4, Oct. 2003, pp. 704-713.

[20] H. Takata and Y. Tsukuda, "Stall Margin Improvement by Casing Treatment - Its Mechanism and Effectiveness," Journal of Engineering Power, Vol. 1, No. 1, Jan. 1977, pp. 121-133.

[21] Q. Yu, Q. Li and L. Li, “The Experimental Researches on Improving Operating Stability of A Single-Stage Transonic Fan," ASME Turbo Expo, ASME, Amsterdam, The Netherlands, 2002.

[22] R. Emmrich, H. Honen and R Niehuis, "Time Resolved Investigations of an Axial Compressor With Casing Treatment Part 1 - Experiment," ASME Turbo Expo: Power for Land, Sea and Air, ASME, Montreal, Canada, 2007.

[23] R. Emmrich, R. Kunte, H. Honen and R Niehuis, "Time Resolved Investigations of an Axial Compressor With Casing Treatment Part 2 - Simulation," ASME Turbo Expo: Power for Land, Sea and Air, ASME, Montreal, Canada, 2007.

[24] W. Lin, K. Sreenivas, R. S. Webster, and D. G. Hyams, "Effect of Casing Groove Locations on the Performance of an Axial-Flow Stage," Joint Propulsion Conference, AIAA, San Jose, CA, 2013. 
[25] W. Lin, K. Sreenivas, R. S. Webster, and D. G. Hyams, "Effect of Casing and Tip Modifications on the Performance of an Axial-Flow Stage," Aerospace Sciences Meeting, AIAA, Nashville, TN, 2012.

[26] D. G. Hyams, R. S. Webster, and K. Sreenivas, "A Generalized Interpolative Interface for Parallel Unstructured Field Solvers," 40th Fluid Dynamics Conference and Exhibit, AIAA, Chicago, IL, June 2010.

[27] D. G. Hyams, R. S. Webster, N. Currier, and K. Sreenivas, "A Generalized, Unstructured Interpolative Interface Method for Rotor-Stator Interaction," 20th AIAA Computational Fluid Dynamics Conference, AIAA, Honolulu, HI, June 2011.

[28] Woodward, R. P., "Comparison of Far-Field Noise for Three Significantly Different Model Turbofans," 46th Aerospace Sciences Meeting and Exhibit, AIAA, Reno, NV, Jan. 2008.

[29] M. D. Collao, R. S. Webster, K. Sreenivas, and W. Lin, "Computational Study of the Effects of Protruding Studs Casing Treatment on the Performance of an Axial Transonic Turbofan," 52nd AIAA/SAE/ASEE Joint Propulsion Conference, AIAA, Salt Lake City, UT, 2016.

[30] Taylor, L. K., Unsteady Three-Dimensional Incompressible Algorithm Based on Artificial Compressibility, Ph.D. thesis, Mississippi State University, MS, 1991.

[31] W. R. Briley, L. K. Taylor, and D. L. Whitfield, "High-Resolution Viscous Flow Simulations at Arbitrary Mach Number," Journal of Computational Physics, Vol. 184, No. 1, 2003, pp. 79-105.

[32] Blazek, J., Computational Fluid Dynamics: Principles and Applications, Elsevier, 2001.

[33] P. R. Spalart and S. R. Allmaras, "A One-Equation Turbulence Model for Aerodynamic Flows," 30th Aerospace Sciences Meeting and Exhibit, AIAA, Reno, NV, 1992.

[34] Hyams, D. G., An Investigation Of Parallel Implicit Solution Algorithms For Incompressible Flows On Unstructured Topologies, Ph.D. thesis, Mississippi State University, Mississippi, May 200.

[35] Pointwise, "Pointwise, Mesh Generation Software for CFD," 2016, Ver. 17.3R4.

[36] Intelligent Light, "Fieldview, Visualization Software," 2016, Ver. 15.0.

[37] GNU, “GNUplot,” 2016, URL: http://www.gnuplot.info. 


\section{VITA}

Max David Collao (David) is originally from Peru. He was born in Lima, the capital, on September 30th, 1984. His parents are Maximo Odon Collao Lopez and Gloria Flor Aldave Ayala. David is the oldest of four children. His siblings are Max Joel, Max Jairo, and Gloria Elisabet. David attended “CP La Reforma” for kinder garden, “CPM 2049” for primary school, and "CNMx San Felipe" for secondary school. He grew up playing with legos, destroying old toys to make new ones, loving buses, enjoying music and dreaming. He used to dream of becoming either an inventor or a business manager/owner. He started attending college at "Universidad Nacional del Callao," majoring in business management, but dropped it to go to college in the United States.

David came to the United States on January 9th, 2004. He started attending college four days after his arrival, at Lipscomb University in Nashville, TN. He graduated from Lipscomb in the Spring of 2009 earning a Bachelors degree in Mechanical Engineering with a Minor's degree in Applied Math. Then, he came to Chattanooga, TN, to work on a Master's degree in Computational Engineering at The University of Tennessee at Chattanooga (UTC). After concluding in the Summer of 2011 he took a break of a few months and returned to UTC in January of 2012 to earn a PhD degree, also in Computational Engineering. His $\mathrm{PhD}$ studies are coming to a conclusion in the Spring of 2017, and now he is eagerly looking forward to be a productive member of society and the corporate world. 
David is a terrible soccer player but a very decent dancer. He speaks Spanish, English, and is currently learning German. Every now and then he still enjoys putting together simple gadgets. David loves Peru but he also loves America, Chattanooga, and Nashville. His favorite dishes are "Lomo Saltado," "Escabeche de Pollo" (with lots of sauce) and, of course, a good burger. Finally, David enjoys spending time with friends and learning about new cultures. 